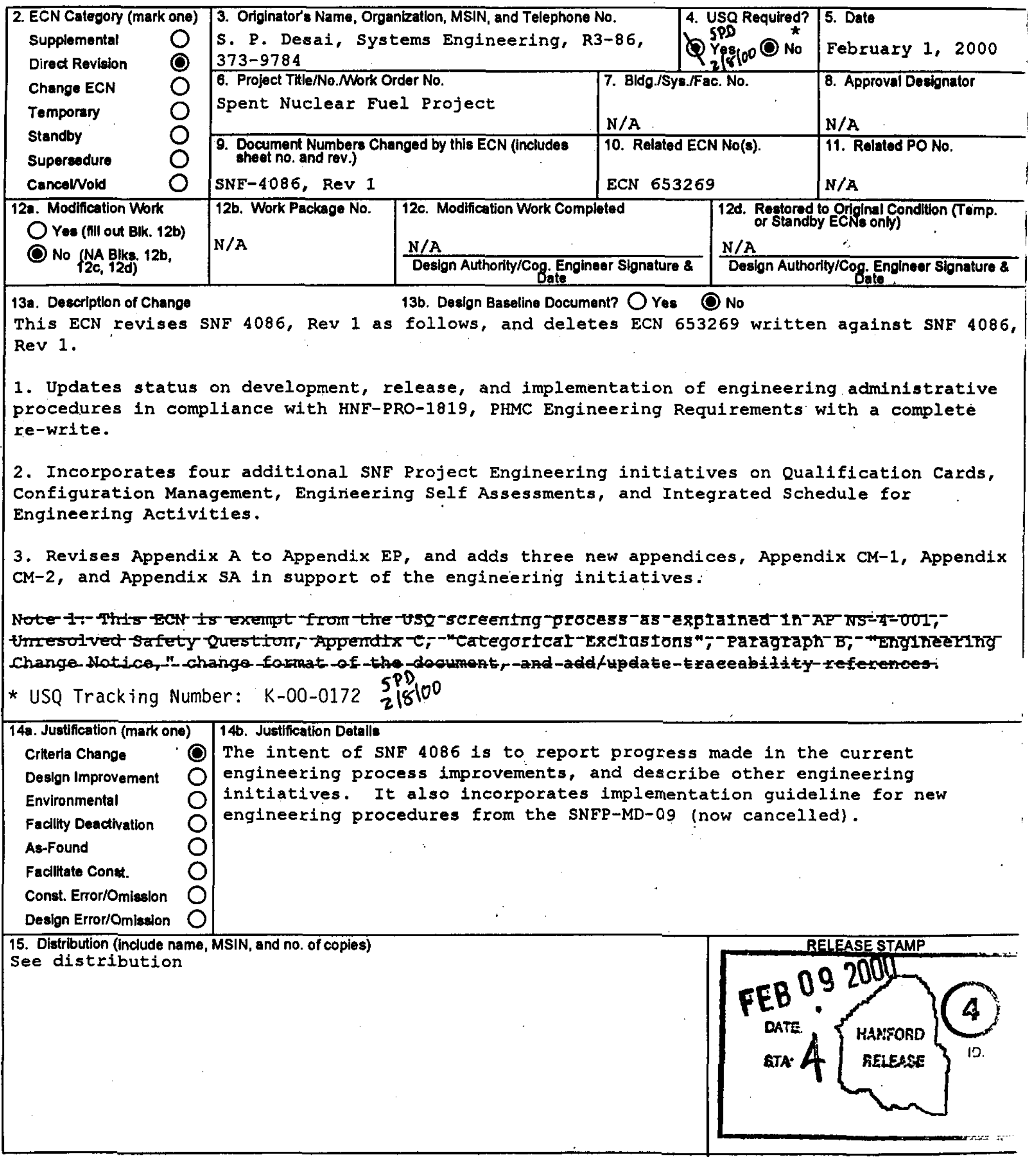


ENGINEERING CHANGE NOTICE

16. Design Verification Required

OYes

( No
Page 2 of 2

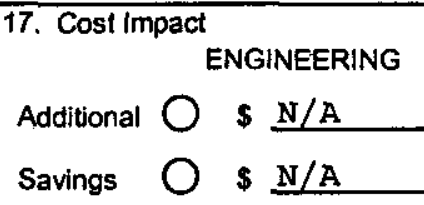

CONSTRUCTION

Additional $\bigcirc \$ \underline{N / A}$

Savings $O \leqslant \underline{N / A}$
1. ECN (use no. from pg. 1)

658185

18. Schedule Impact (days)

improvement $\mathrm{O}$ N/A

Delay

$\mathrm{N} / \mathrm{A}$

19. Change Impact Review: Indicate the related documents (other than the engineering documents identified on Side 1) that will be affected by the change described in Block 13. Enter the affected document number in Block 20.

$\begin{array}{lll}\text { SDD/DD } & \text { Seismic/Stress Analysis } \\ \text { Functional Design Criteria } & \text { Stress/Design Report } \\ \text { Operating Specification } & \text { Interface Control Drawing } \\ \text { Criticafity Specification } & \text { Calibration Procedure } \\ \text { Conceptual Design Report } & \text { Installation Procedure } \\ \text { Equipment Spec. } & \text { Maintenance Procedure } \\ \text { Const. Spec. } & \text { Engineering Procedure } \\ \text { Procurement Spec. } & \text { Operating Instruction } \\ \text { Vendor Information } & \text { Operational Safety Requirement } \\ \text { OM Manual } & \text { IEFD Drawing } \\ \text { FSAR/SAR } & \text { Cell Arrangement Drawing } \\ \text { Safety Equipment List } & \text { Essential Material Specification } \\ \text { Radiation Work Permit } & \text { Fac. Proc. Samp. Schedule } \\ \text { Environmental Impact Statement } \\ \text { Environmental Report } \\ \text { Environmental Permit }\end{array}$

\begin{tabular}{ll}
$\square$ & Tank Calibration Manual \\
$\square$ & Health Physics Procedure \\
$\square$ & Spares Multiple Unit Listing \\
$\square$ & Test Procedures/Specification \\
$\square$ & Component Index \\
$\square$ & ASME Coded Item \\
$\square$ & Human Factor Consideration \\
$\square$ & Computer Software \\
$\square$ & Electric Circuit Schedule \\
$\square$ & ICRS Procedure \\
$\square$ & Process Control Manual/Plan \\
$\square$ & Process Flow Chart \\
$\square$ & Purchase Requisition \\
\hline & Tickler File \\
\hline & N/A
\end{tabular}

$\square$
$\square$
$\square$
$\square$
$\square$
$\square$
$\square$
$\square$
$\square$
$\square$
$\square$
$\square$
$\square$
$\square$
$\square$
$\square$

20. Other Affected Documents: (NOTE: Documents listed below will not be revised by this ECN.) Signatures below indicate that the signing organization has been notified of other affected documents listed below.

Document Number/Revision

N/A
Document Number/Revision

Document Number/Revision

N/A

21. Approvals
Design Authority W.C. Miller
Cog. Eng. S.P. Desai S.P.
Cog. Mgr. G.D. Forehand
QA
Safety $\quad$ N/A
Environ. N/A
Other $\quad$ N/A

Design Agent N/A

PE $\quad \underline{N} / \mathrm{A}$

QA $\quad$ N/A

Safety $\underline{N} / \mathbf{A}$

Design N/A

Environ. N/A

Other $\quad$ N/A

\section{DEPARTMENT OF ENERGY}

Signature or a Control Number that tracks the Approval Signature

N/A

ADDITIONAL

N/A 


\section{CORRESPONDENCE DISTRIBUTION COVERSHEET}

Author

S. P. Desai
Addressee

Distribution
Correspondence No. 00-SNF/SPD-001

February 9,2000

Subject: SPENT NUCLEAR FUEL PROJECT - SNF-4086, REV. 2, ECN 658185

\section{DISTRIBUTION}

Page 1 of 4

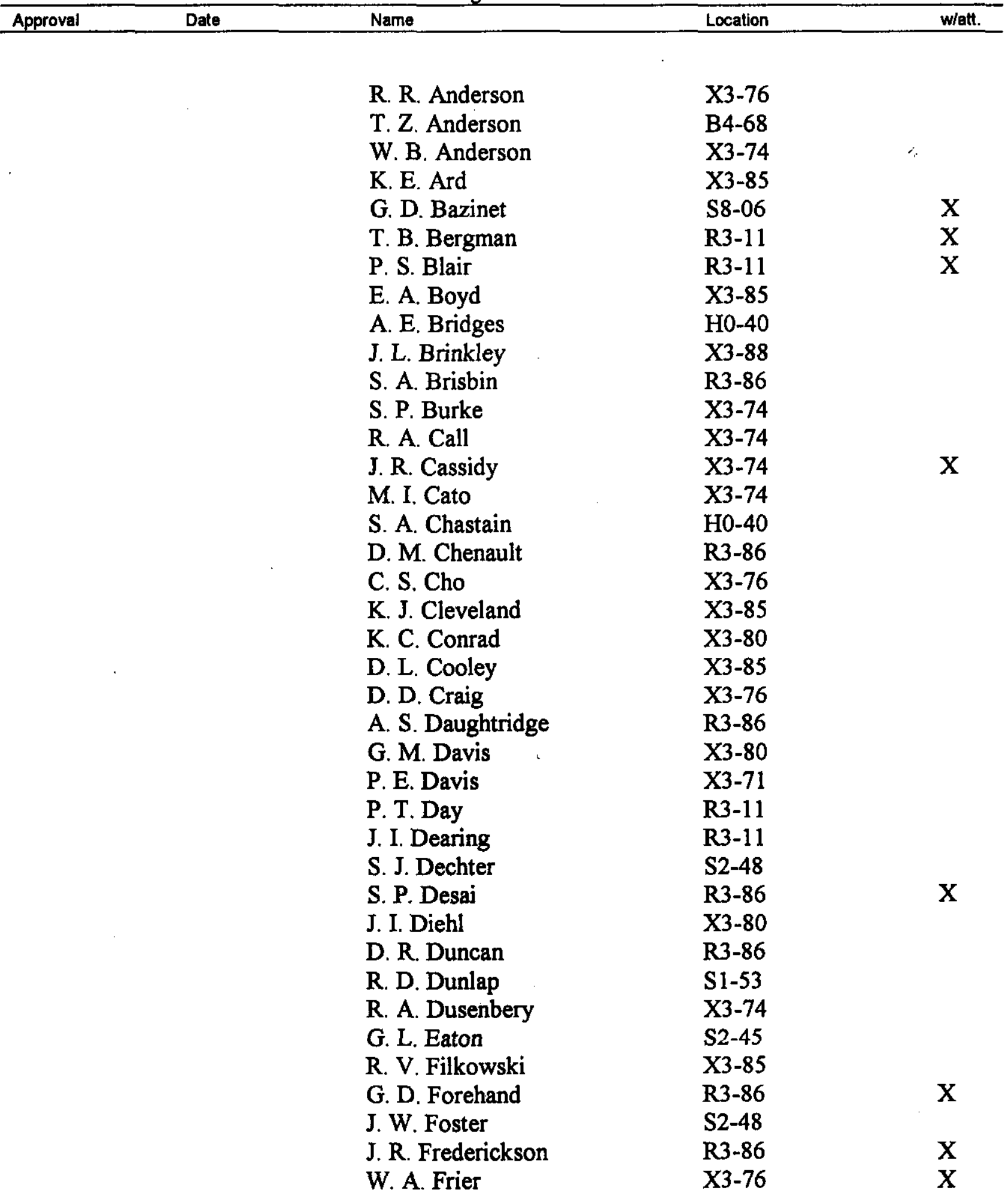


CORRESPONDENCE DISTRIBUTION COVERSHEET

Author

S. P. Desai
Addressee

Distribution
Correspondence No. 00-SNF/SPD-001

February 9,2000

Subject: SPENT NUCLEAR FUEL PROJECT - SNF-4086, REV. 2, ECN 658185

DISTRIBUTION

Page 2 of 4

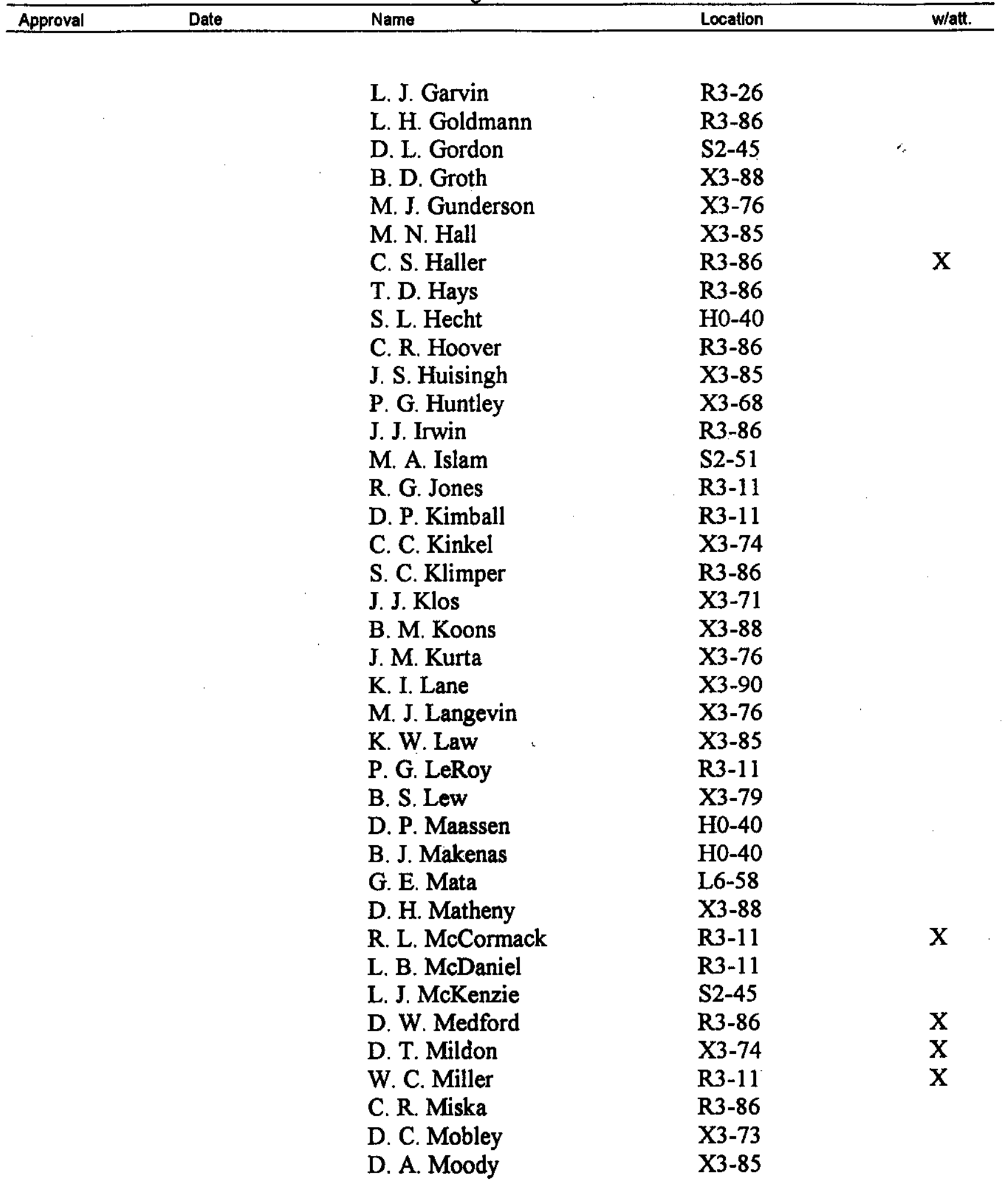




\section{CORRESPONDENCE DISTRIBUTION COVERSHEET}
Author
Addressee
S. P. Desai
Distribution

Correspondence No.

00-SNF/SPD-001

February 9, 2000

Subject: $\quad$ SPENT NUCLEAR FUEL PROJECT - SNF-4086, REV. 2, ECN 658185

\section{DISTRIBUTION}

Page 3 of 4

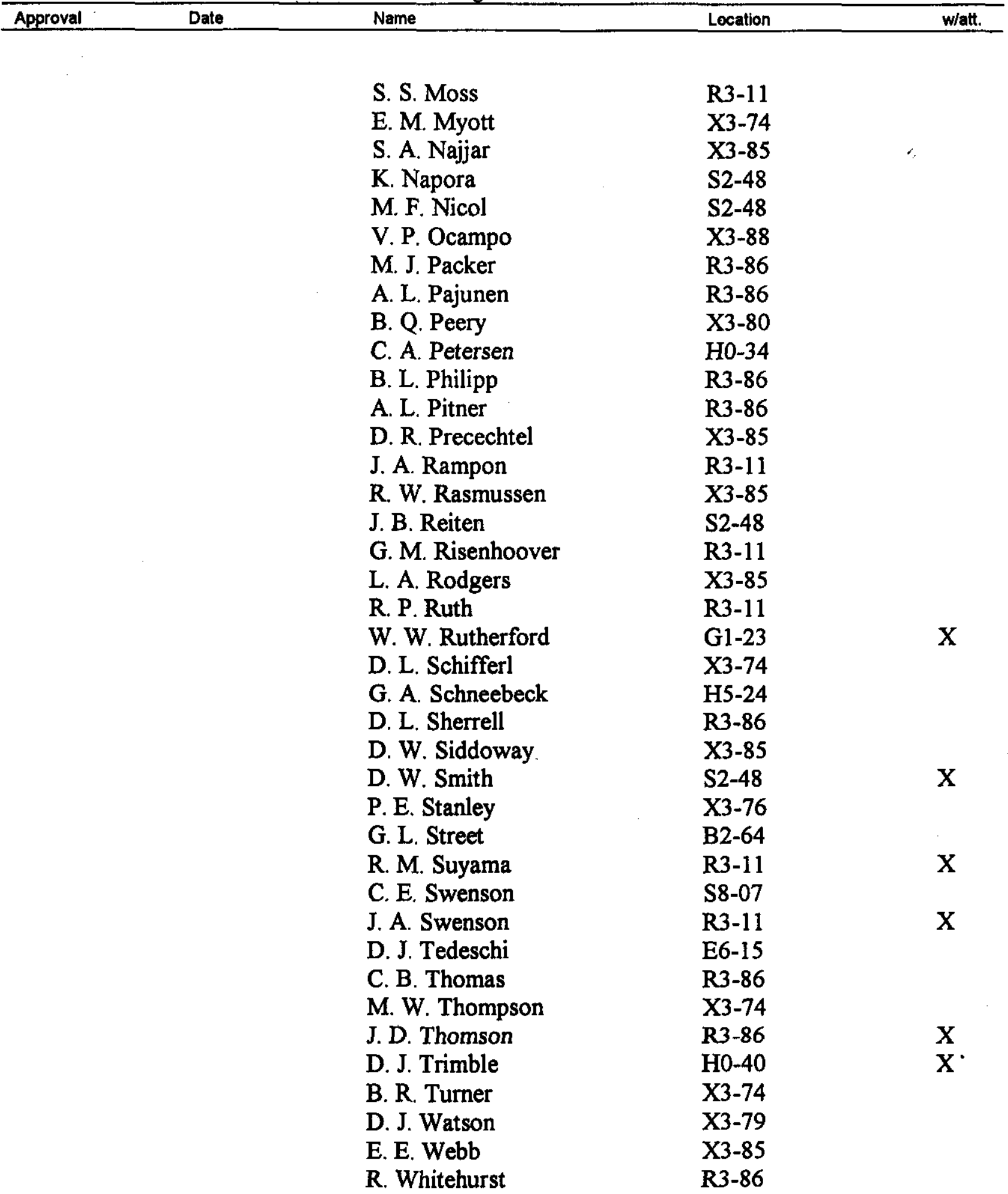




\section{CORRESPONDENCE DISTRIBUTION COVERSHEET}

Author

S. P. Desai
Addressee

Distribution
Correspondence No. 00-SNF/SPD-001

February 9, 2000

Subject: $\quad$ SPENT NUCLEAR FUEL PROJECT - SNF-4086, REV. 2, ECN 658185

\section{DISTRIBUTION}

Page 4 of 4

Approval

Date

Name

Location

w/att.

J. H. Wicks

M. J. Wiemers

R. B. Wilkinson

M. L. Willadsen

R. B. Willard

R. M. Yanochko

W. L. Zickuhr
$\mathrm{X} 3-71$

R3-11

R3-11

$\mathrm{X} 3-85$

R3-11

R3-86

S2-51 


\title{
SNF PROJECT ENGINEERING PROCESS IMPROVEMENT PLAN
}

\author{
Fluor Hanford
}

Richland, WA 00352

U.S. Department of Energy Contract DE-AC06-96RL13200

\author{
EDT/ECN: 658185 \\ UC: UC-510 \\ Ong Code: $\operatorname{men} 400$ \\ Charge Code: 105353 \\ B\&R Code: EW04J1110 \\ Total Pages: $6+71$
}

Key Words: Engineering, Improvement, Initiat1ves, Procedures, Process, GNE-PRO-1819, Configuration Nanagement, Qualification Cards, Mnagement Assessments, Integrated Schedule

Abstract: This plan documents the SNF Project activities and plans to support 1ts engineering process. It describes five SNF Project Engineering initiatives: new engineering procedures, qualification cards process, configuration management, engineering self assessments, and integrated schedule for engineering activities.

TRADEMARK DISCLAIMER. Reference herein to any specilic commercial product, process, or eervice by trade name, tradomark, manufacturer, or otherwise, does not necesearly constitute or imply lis endoresment, recommendation, or fuvoring by the United States Covernment or any agency thereof or lts contractors or subcontractors.

Printed in the United States of America. To obtain coples of this document, contact: Document Control Services, P.O. Box es0, Malletop Ho-08, Richland WA 99352, Phone (509) 372-2420; Fax (509) 376-4080.
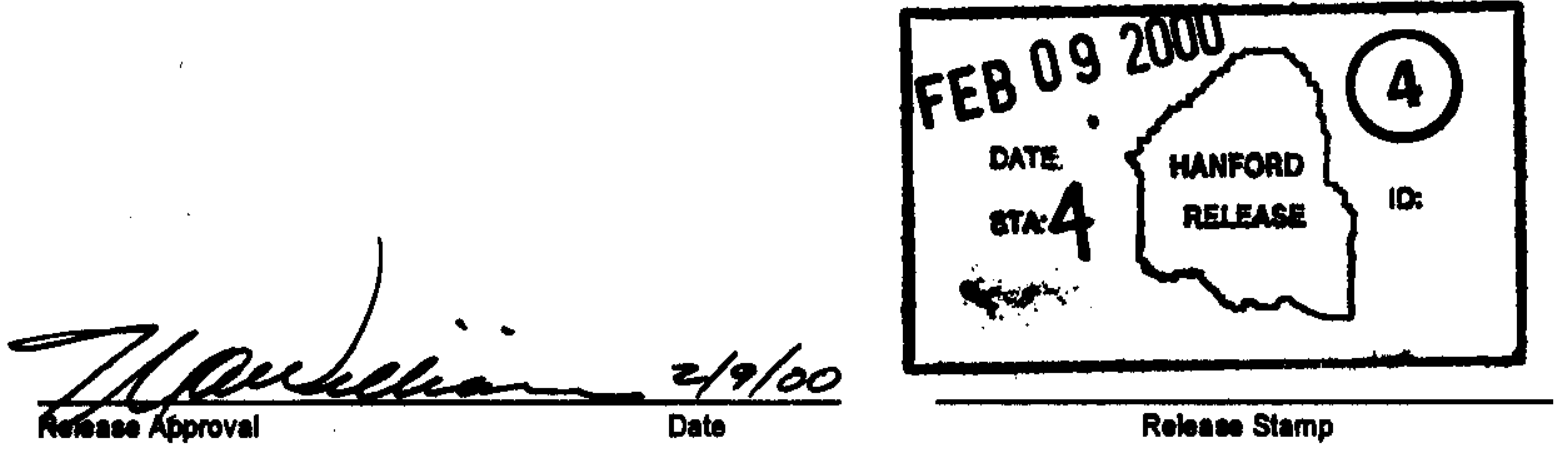
RECORD OF REVISION

(2) This

SNE PROJECT ENGINEERING PROCESS IMPROVEMENT PLAN

Change Control Record
(3) Rovidion
${ }^{2} R S$
(1) Document Number

SNF-4086
Page 1

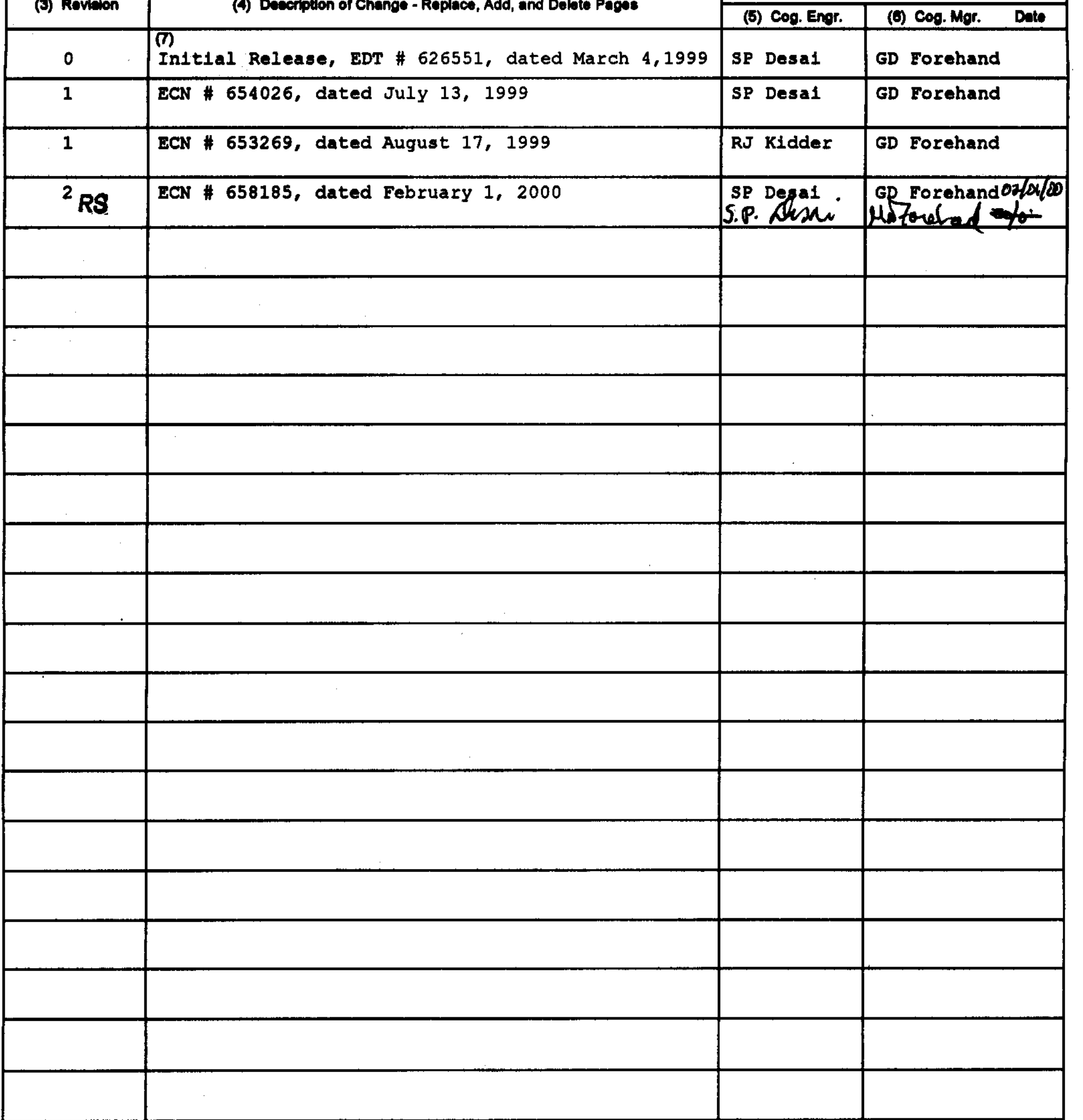


SNF-4086, Rev. 2

SNF PROJECT ENGINEERING PROCESS IMPROVEMENT PLAN

January 2000 


\section{CONTENTS}

$\begin{array}{ll}1.0 & \text { Introduction and Purpose }\end{array}$

$\begin{array}{ll}\text { 2.0 SNF Project Engineering Initiatives } & 1\end{array}$

2.1 Engineering Procedures 1

2.1.1 Background 1

2.1.2 Methodology 1

2.1.3 Status 2

2.1.4 Implementation Strategy for New Engineering Procedures

2.2 Qualification Cards 3

2.2.1 Background $\quad 3$

2.2.2 Methodology 3

2.2.3 Status 3

2.2.4 Implementation Strategy 3

2.3 Configuration Management 4

2.3.1 Background 4

2.3.2 Methodology 4

2.3.3 Status 4

2.3.4 Implementation Strategy 5

2.4 Engineering Self Assessments 5

2.41 Background 5

2.4.2 Methodology 5

2.4.3 Status 5

2.4.4 Implementation Strategy 5

$\begin{array}{lll}\text { 2.5 Integrated Schedule for Engineering Activities } & 6\end{array}$

$\begin{array}{lll}3.0 & \text { References } & 6\end{array}$

\section{LIST OF APPENDICES}

Appendix EP: New Engineering AP Compliance to HNF-PRO-1819 Requirements Appendix CM-1: Status of Improvement Plan Resulting from the CM Gap Analysis Appendix CM-2: SNF Project Closure/Status on Response FDH-9953258A R2 to RL Assessment 99-AMW-015

Appendix SA: SNF Engineering Self-Assessment Schedule 


\section{LIST OF TERMS}

SNF Spent Nuclear Fuel

APs Administrative Procedures

PHMC Project Hanford Management Contract

PHMS Project Hanford Management System 


\section{SNF-4086, REV. 2 \\ SNF PROJECT ENGINEERING PROCESS IMPROVEMENT PLAN}

\subsection{INTRODUCTION AND PURPOSE}

This Engineering Process Improvement Plan documents the SNF Project activities and plans to support its engineering process. It describes major initiatives undertaken or to be pursued by the SNF Project Engineering organization.

A major transition of the engineering resources to support the SNF Project operations phase is anticipated during FY 2000. Revisions to this plan will be developed as these initiatives are completed and the Engineering organization is transitioned from its current configuration to a technical support role for the operations phase.

\subsection{SNF PROJECT ENGINEERING INITIATIVES}

This section describes the initiatives in detail to provide the background, methodology, status, implementation strategy, and point of contact (POC).

\subsection{Engineering Procedures}

\subsubsection{Background}

The HNF-PRO-1819, PHMC Engineering Requirements became effective March 1, 1999. Prior to the effective date of HNF-PRO-1819, the SNF Project performed engineering work according to HNF-1613, SNF Project Engineering Practices. Full compliance with HNF-PRO-1819 required extensive changes to the HNF-1613 procedures. Therefore, as an interim measure, the SNF Project Director issued a management directive, SNFP-MD-009, Implementation Of PHMC Engineering Requirements on February 26, 1999, to implement requirements of HNF-PRO-1819 until procedures could be revised. This directive validated interim use of HNF-1613 with administrative controls to impose HNF-PRO1819 requirements until September 30, 1999. A new set of engineering procedures in full compliance with HNF-PRO-1819 were to be provided to replace HNF-1613 by September 30, 1999.

\subsubsection{Methodology}

The SNF Project Chief Engineer formed the Procedure Integration (PI) group to expedite development and release of new engineering procedures under a joint leadership of the Deputy, SNF Project Engineering, and Manager, Systems Engineering. The PI members were appointed by the engineering managers based on subject matter expertise. The group met twice a week to formulate an integrated approach, and identify time lines for:

- revisions to topic-specific procedures complying with HNF-PRO-1819 requirements,

- development of new procedures,

- tracking the review and approval cycle for each procedure, and

- releasing the procedures. 
SNF-4086, REV. 2

The PI leadership organized numerous briefing sessions to communicate progress made by the PI group with the SNF Project engineers and technical support staff involved in the SNF Project engineering activities. Initial orientation sessions were held in $200 \mathrm{E}$ and $100 \mathrm{~K}$ Areas to brief the employees regarding SNFP-MD-009 and SNF-4086, Rev 0, SNF Project Engineering Process Improvement Plan. The PI leadership also established an e-mailbox (^SNFP Eng Process Improvements) to announce progressive communications, invite questions or inquiries from the employees, and verify completion of specific mandatory actions. Follow on briefing sessions consisted of presentations made by the procedure authors as these procedures were released. The intent of the briefings was to ensure that the employees were aware of the new procedures, and provide them an opportunity to ask questions of the subject matter experts. A database was established to track required attendance at these briefings.

\subsubsection{Status}

A top level engineering procedure was identified to describe engineering process for the SNF Project (AP EN-6-030, Engineering Process.) This procedure contains a roadmap in an appendix to indicate in a matrix which procedure addresses each of the HNF-PRO-1819 requirements.

The SNFP-MD-009 and HNF-1613 were withdrawn on September 30, 1999 with the release of new and revised SNF Project Engineering procedures to comply with HNF-PRO-1819. These procedures are available as Administrative Procedures (APs) in the most recent version with a following path:

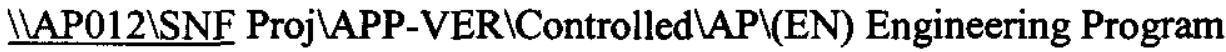

Appendix EP describes the released procedures in a summary form including the HNF-PRO-1819, Rev 3 requirements each procedure meets. This is to provide status when this appendix was updated. Since the procedures are subject to continuous reviews during use, updates are identified when process improvements are necessary. This is due to changing nature of engineering work. The follow on updates is not identified in this appendix.

A major briefing session was held on October 25, 1999 to communicate management expectations for implementation, brief employees on newly released procedures, and provide an opportunity for immediate feedback. Follow-up sessions were held for technical staff unable to attend this first briefing.

\subsubsection{Implementation Strategy for New Engineering Procedures}

The SNF Project is progressing to complete the design and construction phase in many of its subprojects. In some cases, it would be counterproductive to impose newly released engineering procedure requirements retroactively on all engineering activities. At the same time, there are opportunities where these procedures can be implemented so that all current requirements are implemented as described in the procedures. The following guidelines are provided to clarify procedure implementation.

a. For the completed engineering work (e.g., drawings, reports, analysis, verification, testing), no rework will be required for the sole purpose of compliance with the newly released procedures. This guideline presumes that the original work was in compliance with the requirements in effect at the time that engineering activity was completed. 
SNF-4086, REV. 2

b. For the engineering work in progress, the requirements in the newly released engineering procedures must be implemented. For specific instances where this is not feasible, variance(s) to the procedure(s) must be documented, and approved by the cognizant manager and the SNF Project Chief Engineer.

c. For the new engineering work, the most recent engineering procedures must be followed including any subsequent revision(s) to the procedures.

The Deputy, SNF Project Engineering and/or Manager, Systems Engineering should be contacted for any questions or further clarification in this regard.

\subsection{Qualification Cards}

\subsubsection{Background}

The DOE Order 5480.20A, "Personnel Selection, Qualification, and Training Requirements for DOE Nuclear Facilities," contains minimum requirements that must be included in training and qualification programs. The objectives are to ensure the development and implementation of contractor-administered training programs that provide consistent and effective training for personnel at DOE nuclear facilities. In addition, there are Office of Civilian Radioactive Waste Management (OCRWM) requirements (DOE/RW-0333P, "U.S. Department of Energy Office of Civilian Radioactive Waste Management Quality Assurance Requirements and Description for the Civilian Radioactive Waste Management Program, "QARD," Section 2.2.12, and 2.2.13) for Personnel Qualification applicable to the OCRWM work performed within the SNF Project.

\subsubsection{Methodology}

The SNF Project Chief Engineer has established a process to qualify technical personnel in the practice of engineering. This process is described in the ENG-DI-012, "Engineering Qualification." (Draft)

\subsubsection{Status}

The design authorities and cognizant engineers have been qualified and appointed in accordance with the AP EN-6-004, "Engineering Personnel Qualifications, Appointments and Responsibilities." The technical personnel involved in the Phased Startup Initiative (PSI) have also been provisionally qualified. The engineering qualification process described in the ENG-DI-012 is in the final stage of the development.

\subsubsection{Implementation Strategy}

The target date for qualifying engineering managers, design authorities, and cognizant engineers in accordance with ENG-DI-012 is March 2000. The remaining engineering personnel and the technical support staff will be qualified on a schedule to support start of fuel movement operations. This will be initially implemented for current K Basin operational facilities. As systems are turned over for operations, the qualification process will be expanded. 


\subsection{Configuration Management}

\subsubsection{Background}

The configuration management plan (CMP) for the SNF Project is described in HNF-SD-SNF-CM-001. This plan is based on HNF-SD-SNF-RD-001, SNF Project Standards/Requirements Identification Document (S/RID), and the PHMC Configuration Management Plan, HNF-MP-013 and tailored to the needs of the SNF Project. Appendix A in the HNF-SD-SNF-CM-001 is a matrix that links the specific requirements in the CMP to the source document and to the implementing procedures. A gap analysis was performed in December 1998 (Interoffice Correspondence 98-SNF/GDF-015) to determine if all necessary implementing procedures were identified and if the identified procedures addressed the CMP requirements adequately. This analysis resulted in recommendations for removing the identified gaps. Subsequently, a configuration management improvement plan (Interoffice Correspondence 99SNF/GDF-002) was released in March 1999 to address each of the recommendations and assign actions.

The Department of Energy, Richland Operations Office (RL) conducted an integrated assessment of the configuration management program implementation on the SNF Project (Letter 99-AMW-015) in February 1999. The RL assessment identified two concerns, 13 findings, and five observations related to the SNF Project activities.

A software audit (PAD-AUD-99-021, Audit of FDH Computer Software Quality Assurance, issued in July 1999 and conducted by the RL Performance Assessment Division) identified weaknesses in software configuration control. The SNF Project Process Engineering organization took the lead in working with the Records and Information Management (RIM) services to develop software configuration management procedures.

\subsubsection{Methodology}

The Systems Engineering organization in the SNF Project led the effort to respond to the RL assessment. A comprehensive review and evaluation of the RL assessment (Letter FDH-9953258A R2) was conducted in June 1999. This included a determination of root causes of the problems and circumstances leading to the concerns and the finding along with corrective actions for more effective implementation of the CM process.

\subsubsection{Status}

Appendix CM-1 documents the completion of all actions identified in the configuration management improvement (corresponding to the Interoffice Correspondence 99-SNF/GDF-002).

The Letter FDH-9953258A R3 released in December 1999 reported the status or closure of 65 discrete actions identified in FDH-9953258A R2. Appendix CM-2 provides the status of specific actions (an attachment to the Letter FDH-9953258A R3).

New software control procedures are in development to resolve CM issues. Completion and implementation will be established when current budget and staff issues are resolved. 


\subsubsection{Implementation Strategy}

With most of the actions completed, follow-on assessments and surveillances will be used to verify the effectiveness of the corrective actions. A detailed schedule for the FY 2000 assessments is in the development stage pending resolution of current budget and staff allocation issues, and will be integrated with the Management Assessment actions identified in Section 2.4.

\subsection{Engineering Self Assessments}

\subsubsection{Background}

The AP MS-1-036, "Management Assessments," establishes responsibilities, requirements, and processes for planning, scheduling, conducting, and documenting management assessments (MA) within the Spent Nuclear Fuel Project (SNF Project). The SNF Project Chief Engineer is responsible for conducting MAs in the Engineering functional area.

\subsubsection{Methodology}

The AP MS-1-036 describes the process for conducting the MAs.

\subsubsection{Status}

The MAs for FY 2000 have been planned and described in the following section.

\subsubsection{Implementation Strategy}

The Appendix SA provides a summary of self-assessment activities for SNF Engineering and target dates for FY 2000. This includes items selected for the SNF Project Management Assessment activities and less formal self-assessment activities to be considered for inclusion in the integrated schedule (see section 2.5). The approved MA items are provided in Interoffice Correspondence \#99-SNF/RBW-049, Spent Nuclear Fuel Project FY00 Management Assessment Plan Transmittal. As information is acquired from the management self assessments (MSAs), the relevant data will be integrated into the Appendix SA.

The MAs will be controlled through the Engineering working level schedule and the Project integrated schedule. This control will align the MAs with other priorities facing the Project. 


\subsection{Integrated Schedule for Engineering Activities}

\section{SNF-4086, REV. 2}

The SNF project is transitioning construction projects to operations. There are two major subsystems and two other major facilities going through a start-up in FY 2000. As a result, the needs for engineering support are significantly greater than the existing resources available to provide coverage for all the facilities and all the different engineering functions.

The performance of any assessment also requires the expenditure of engineering resources. Given the shortage of engineering resources, a method for prioritizing and controlling work must be instituted and followed, so that the scarce resources are assigned to the highest priority activities. The method for this is usually the project's integrated working level schedule. The lack of such a schedule for the SNF Project has seriously impeded the ability of any of the functional and support groups to prioritize their activities.

As a result, engineering initiated an action to build its own integrated schedule, which included those pieces of engineering activities from every organization which funded and used engineering resources. This schedule is used to determine priorities, schedule and control engineering work.

The SNF project is now in the process of developing its working level integrated schedule. The engineering activities are being rolled into this overall schedule, and will not be scheduled separately from the project schedule. The same method of tracking and controlling work will be used, but using the overall project schedule, to drive priorities will result in a better utilization of resources.

\subsection{REFERENCES}

HNF-PRO-1819, PHMC Engineering Requirements, Rev 3, June 8, 1999

SNFP-MD-009, SNF Project Management Directive on Implementation of PHMC Engineering Requirements

HNF-1613, SNF Project Engineering Practices

AP EN-6-030, Engineering Process

DOE Order 5480.20A, Personnel Selection, Qualification, and Training Requirements for DOE Nuclear Facilities

DOE/RW-0333P, U.S. Department of Energy Office of Civilian Radioactive Waste Management (OCRWM) Quality Assurance Requirements and Description for the Civilian Radioactive Waste Management Program, "QARD"

ENG-DI-012, Engineering Qualification (Draft)

AP EN-6-004, Engineering Personnel Qualifications, Appointments and Responsibilities HNF-SD-SNF-CM-001, SNF Project Configuration Management Plan

HNF-SD-SNF-RD-001, SNF Project Standards/Requirements Identification Document 
HNF-MP-013, PHMC Configuration Management Plan

98-SNF/GDF-015, Interoffice Correspondence, "Configuration Management Plan Gap Analysis", dated December 31, 1998

99-SNF/GDF-002, Interoffice Correspondence, "Configuration Management Improvement Plan", dated March 4, 1999

99-AMW-015, Letter, "Configuration Management Deficiencies on the SNF Project", dated May 10, 1999

PAD-AUD-99-021, “Audit of FDH Computer Quality Assurance”, dated July 1999

FDH-9953258A R2, "Regarding Configuration Management Deficiencies on the SNF Project", dated June 23, 1999

FDH-9953258A R3, "Regarding Configuration Management Deficiencies on the SNF Project", dated December 29, 199

AP MS-1-036, Management Assessments

99-SNF/RBW-049, Interoffice Correspondence, "SNF Project FY00 Management Assessment Plan Transmittal", dated September 15, 1999 


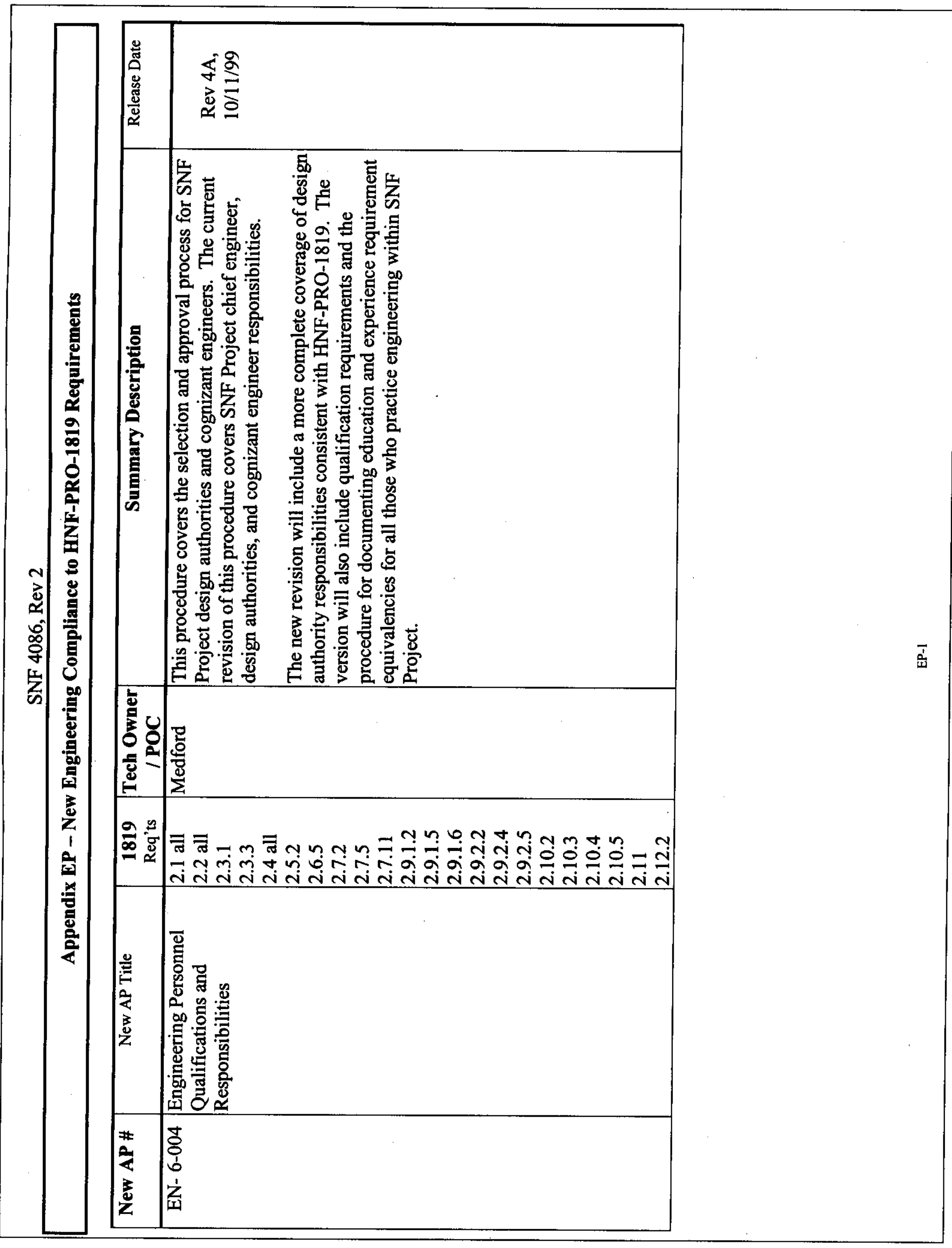




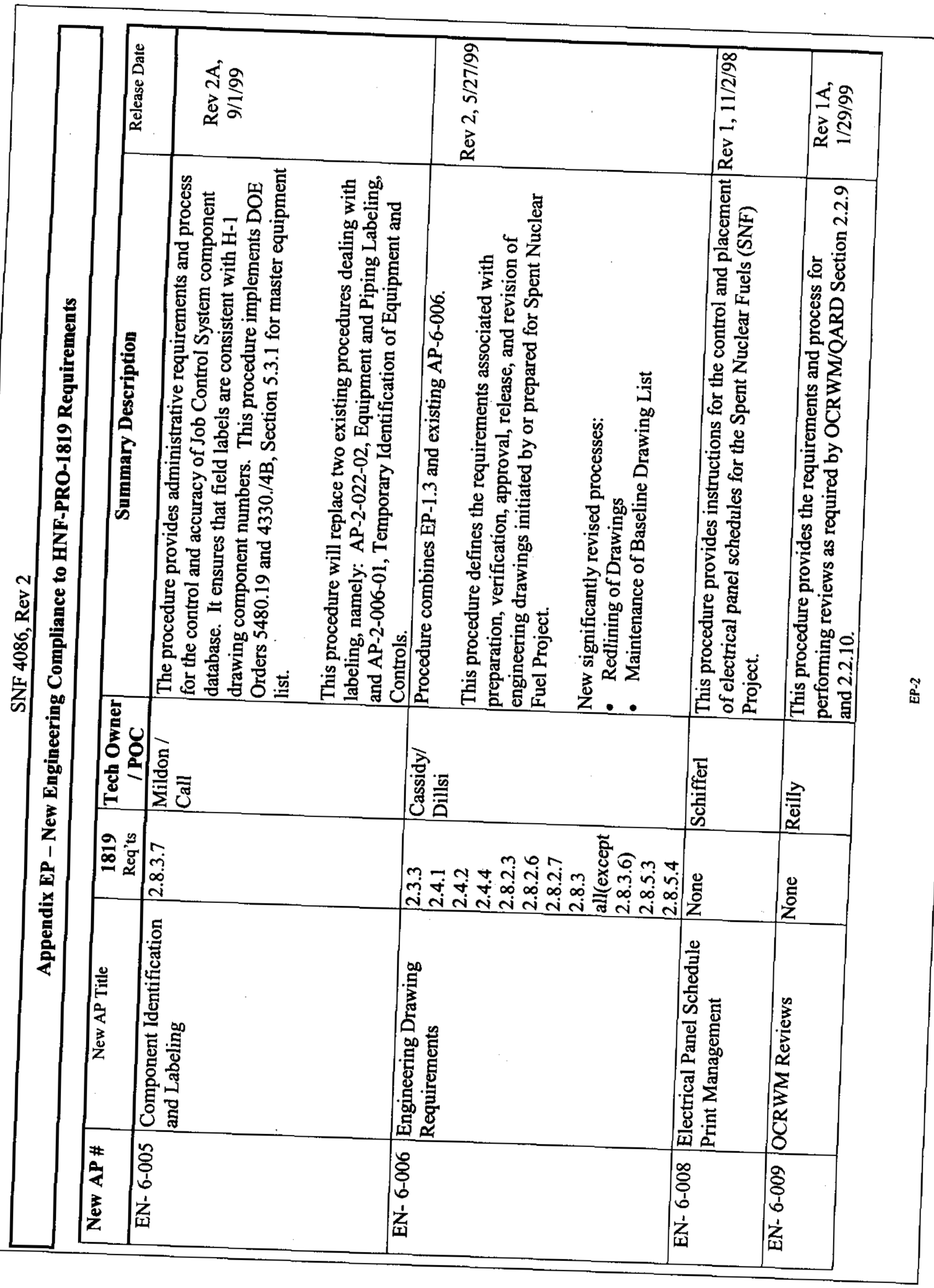




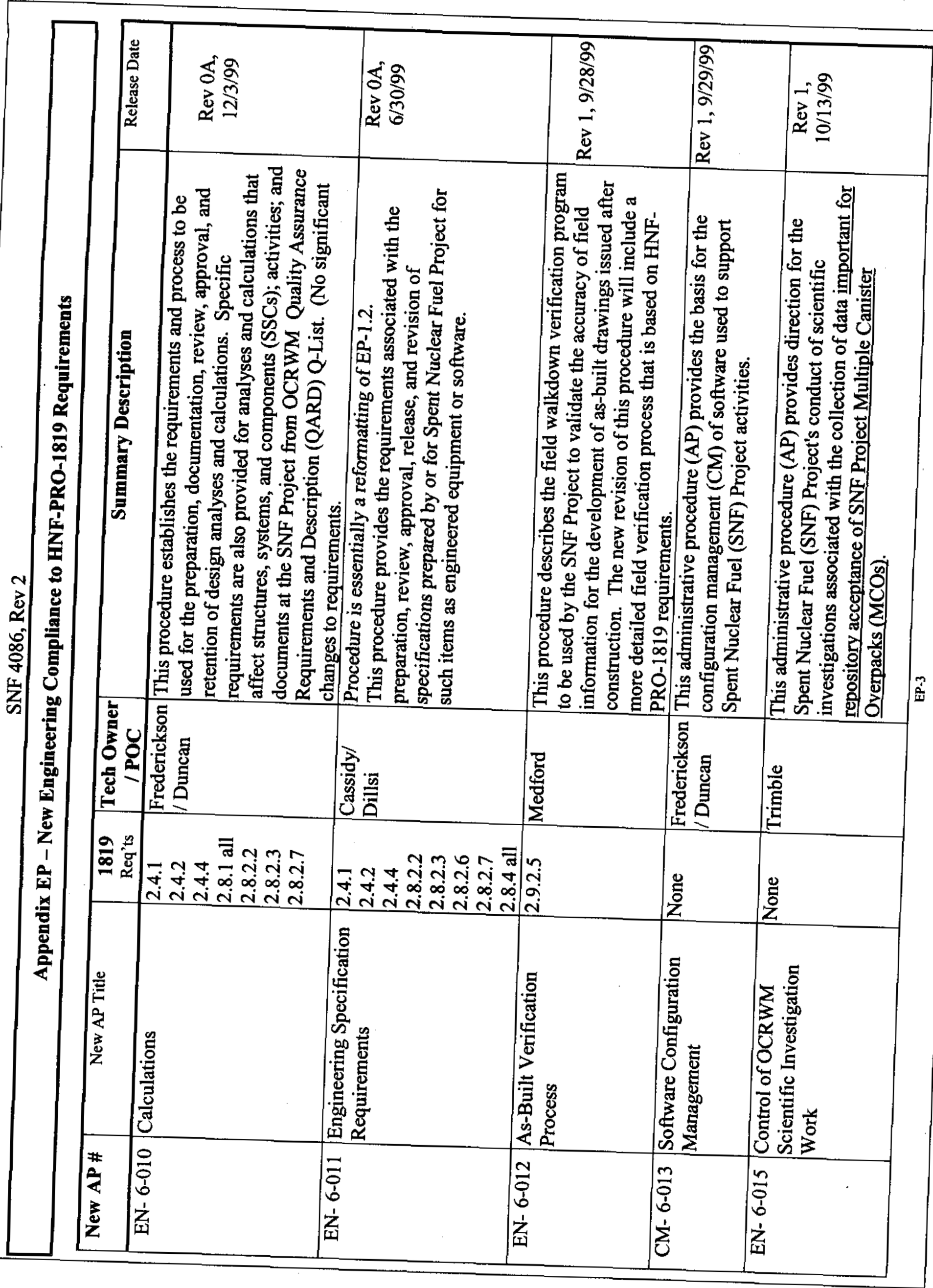




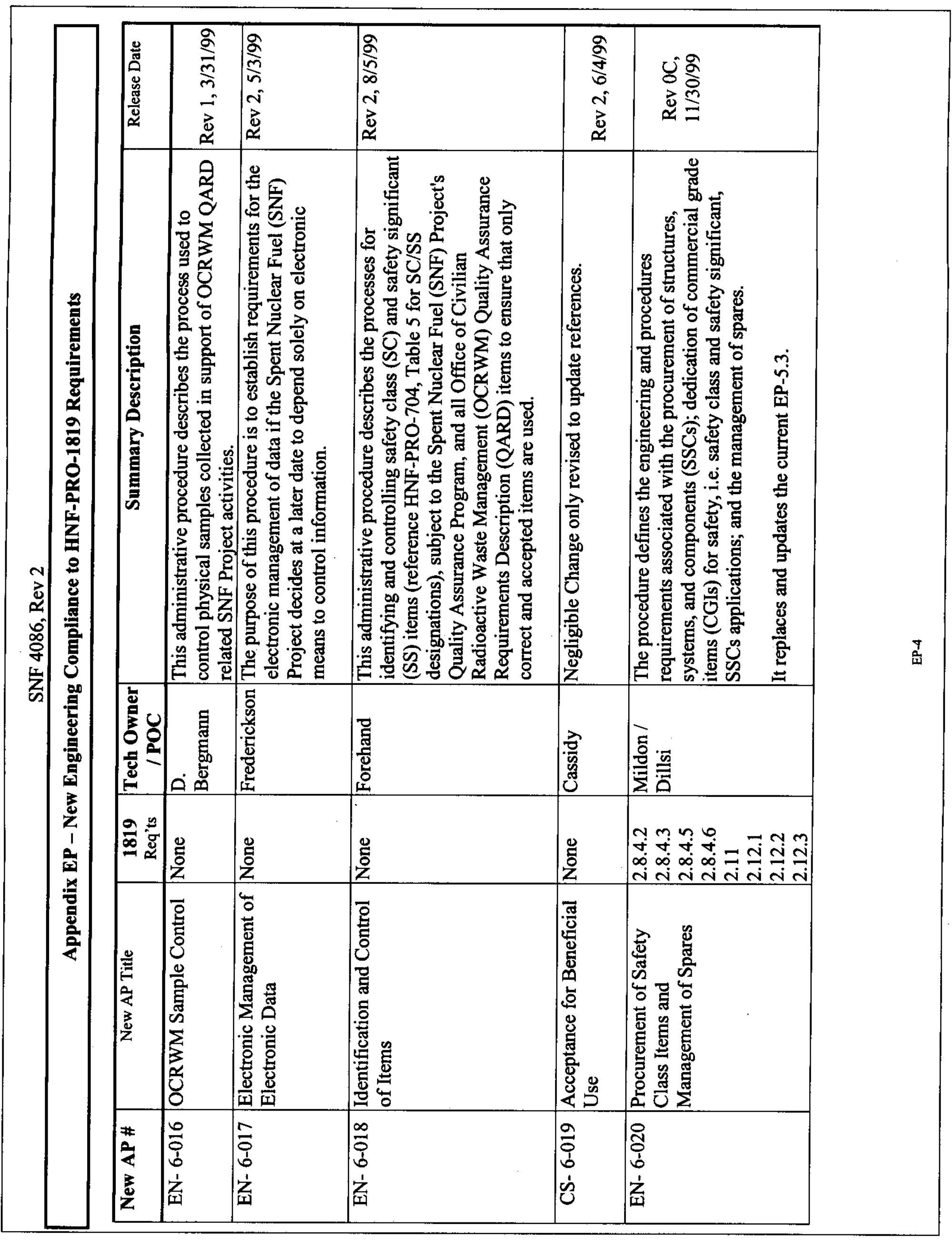




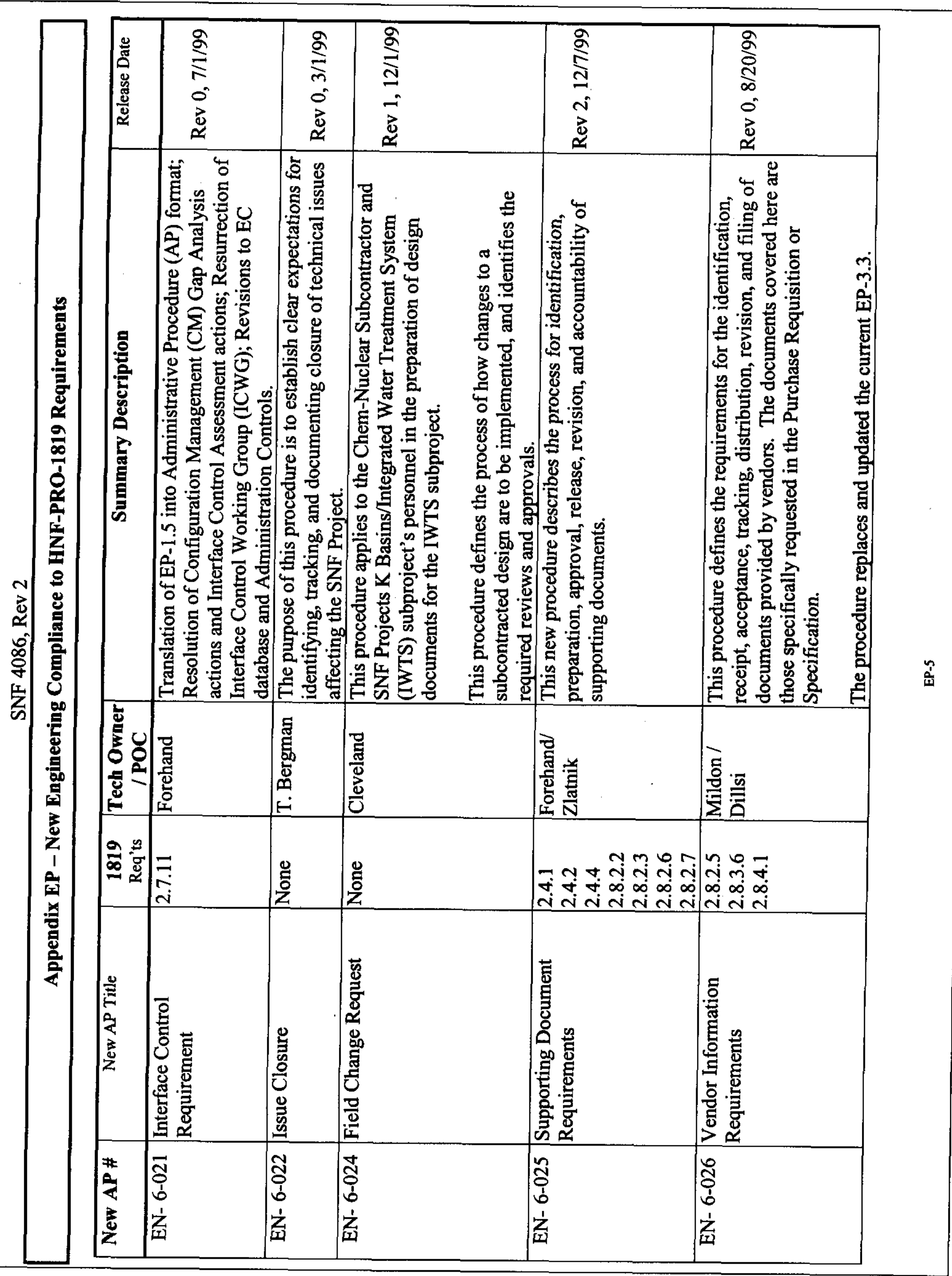




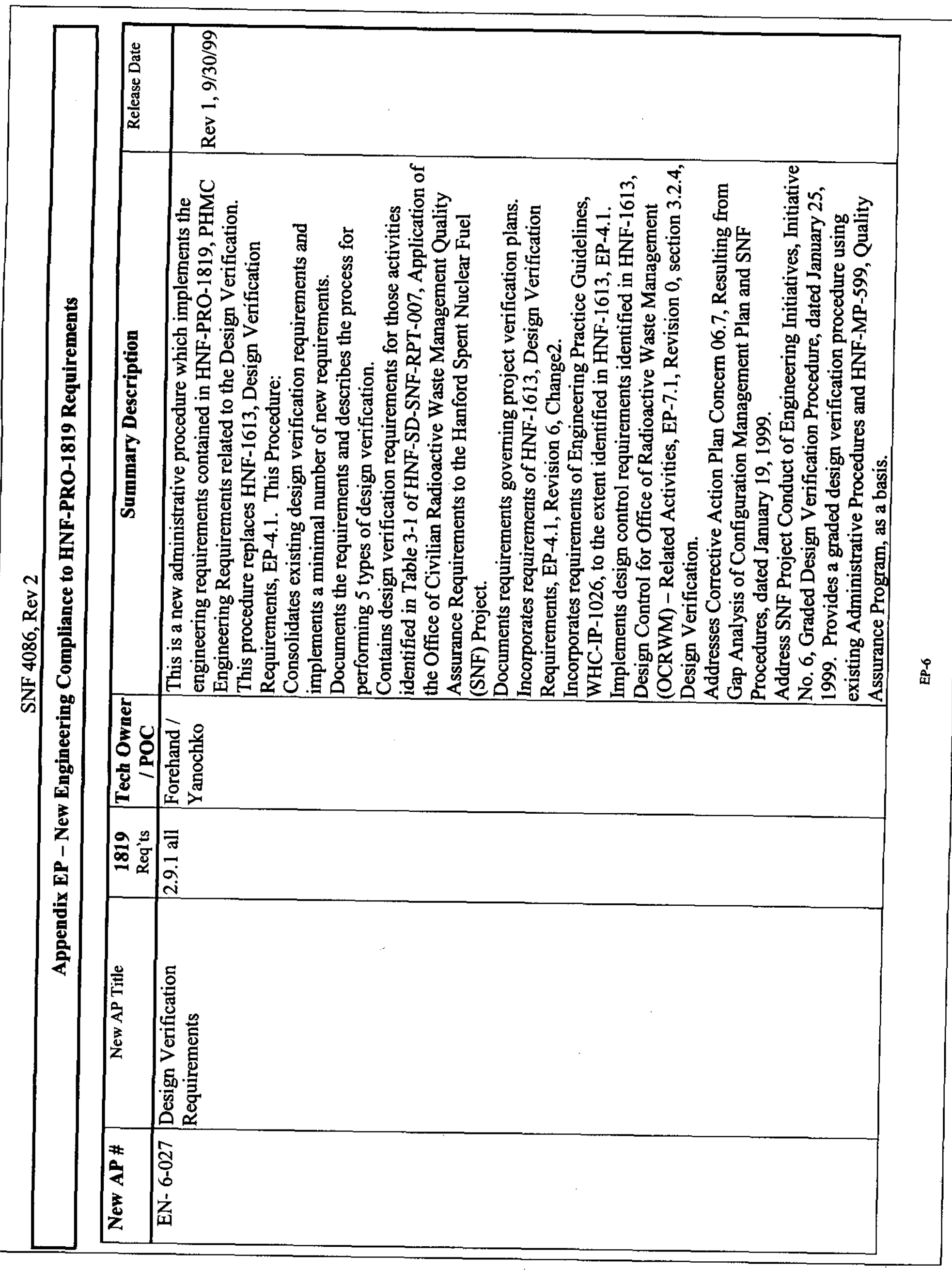




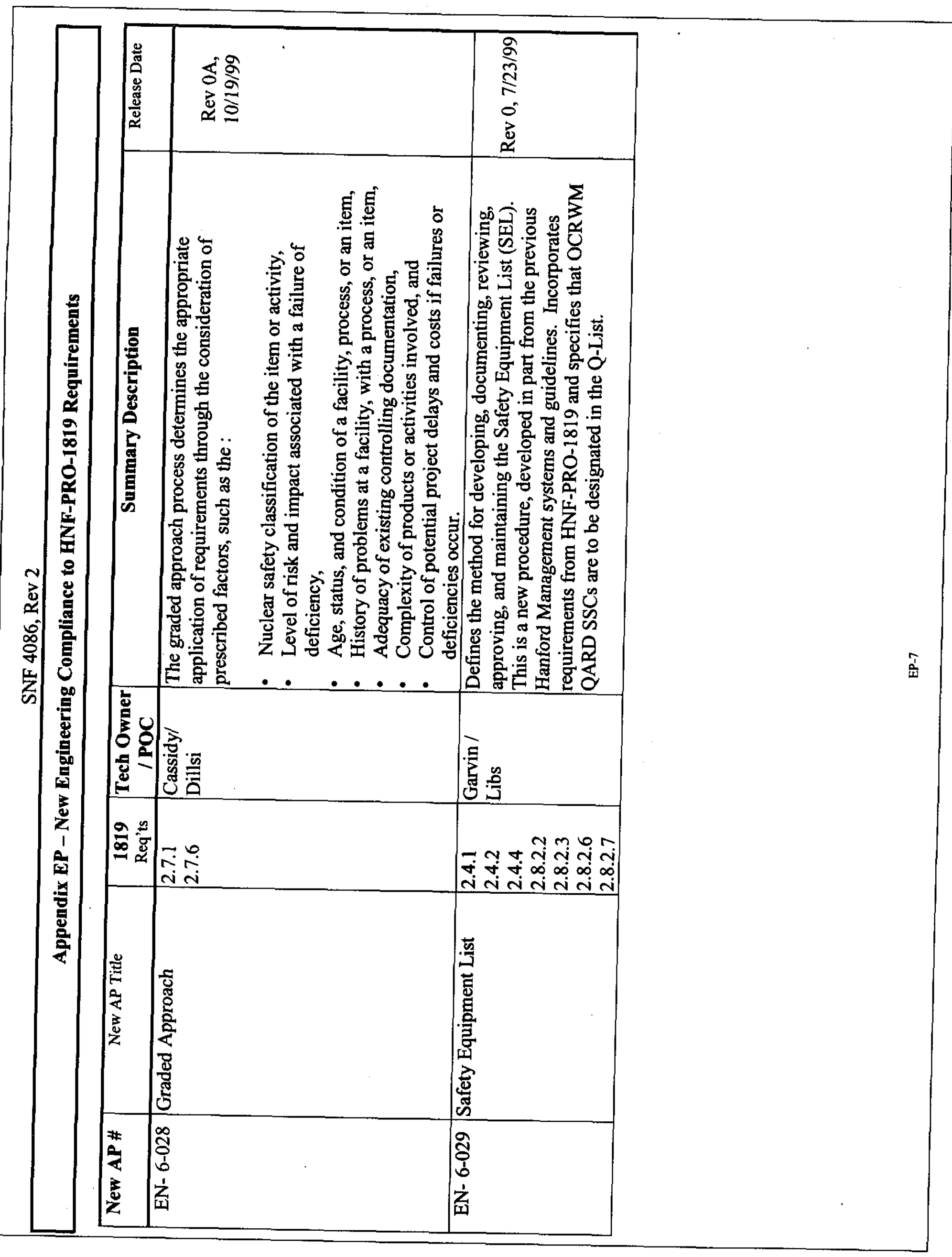




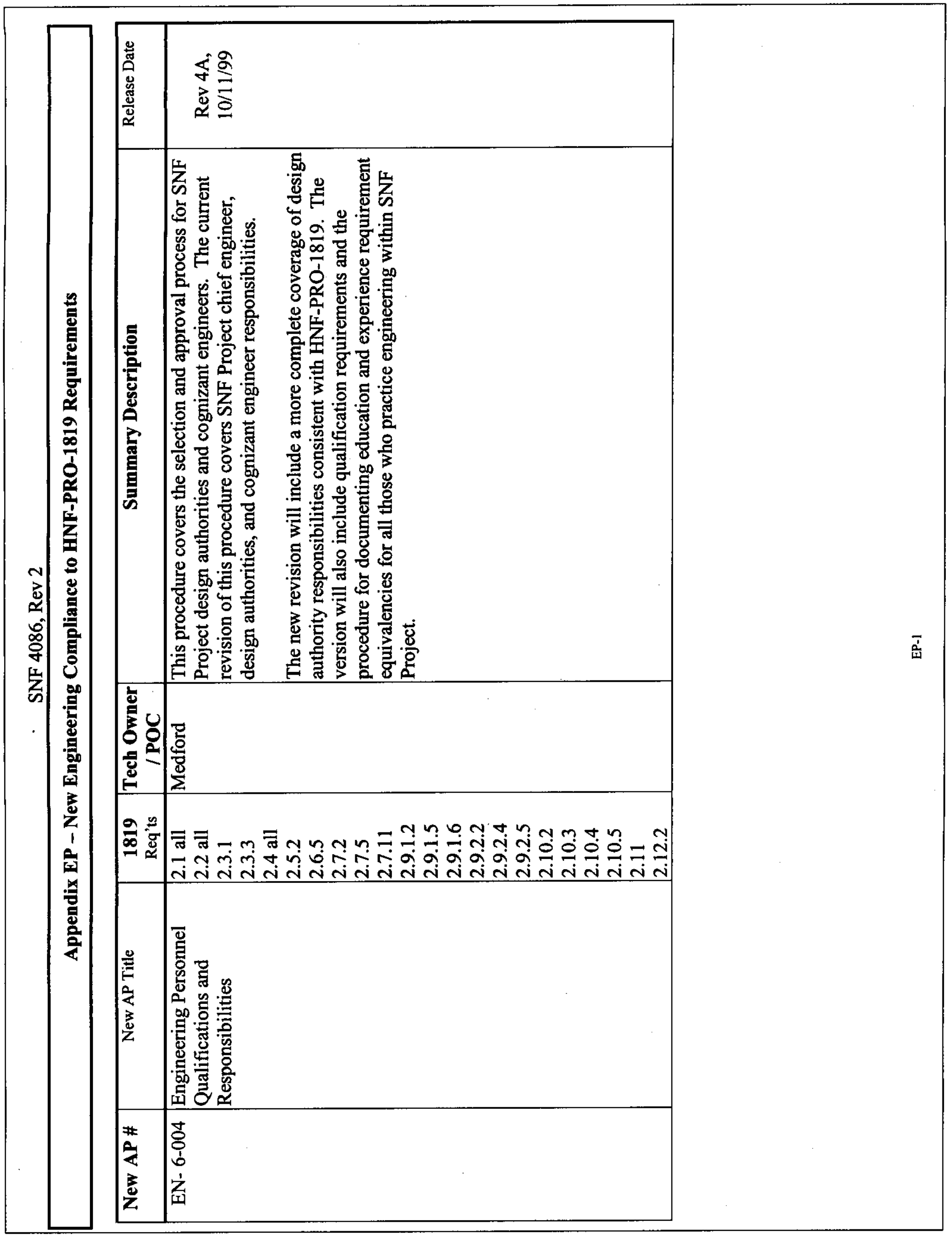




\begin{tabular}{|c|c|c|c|c|c|c|c|c|}
\hline \multirow{6}{*}{ | } & 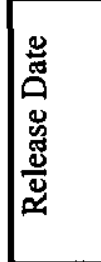 & 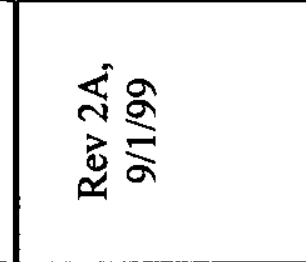 & & 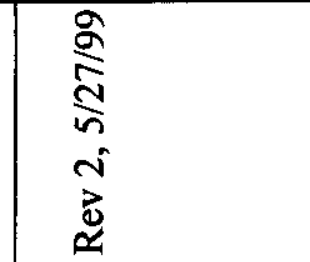 & & 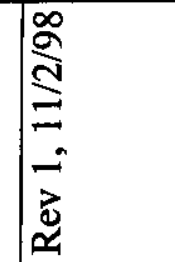 & 达 & \\
\hline & 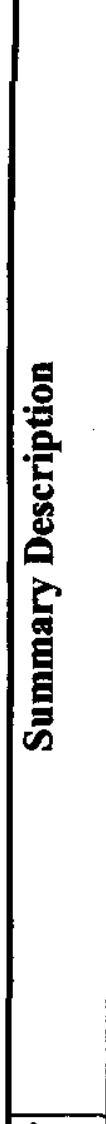 & 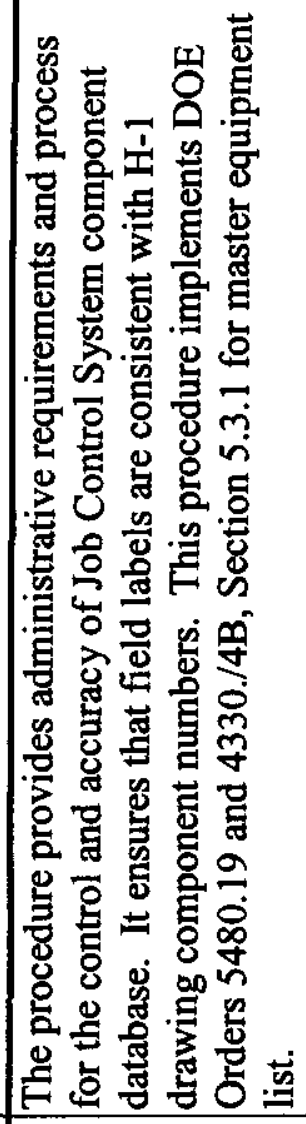 & 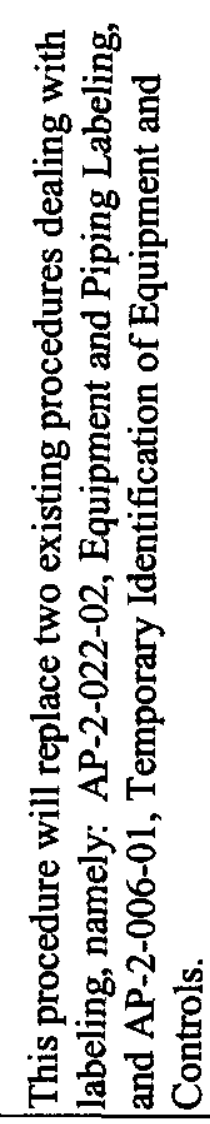 & 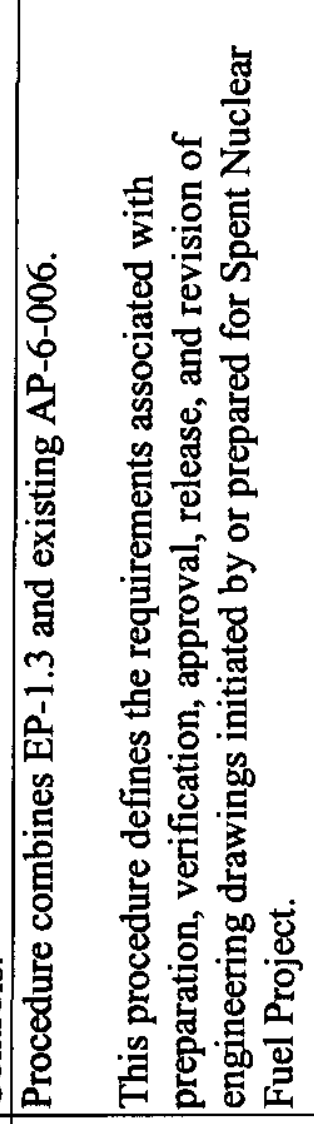 & 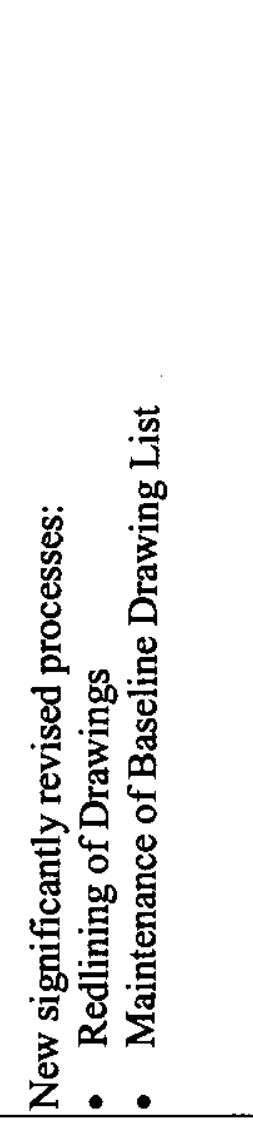 & 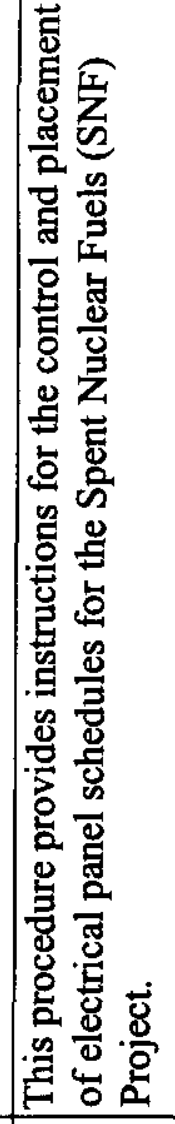 & 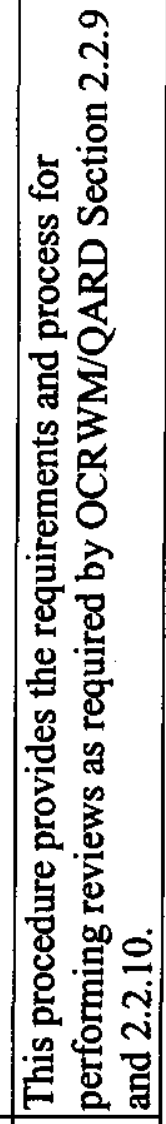 & นับ \\
\hline & 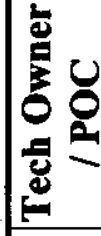 & 这 & & 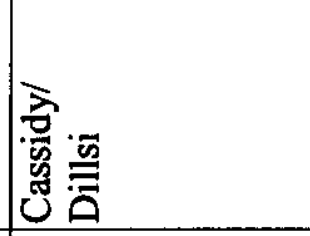 & & 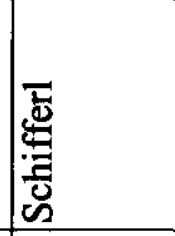 & 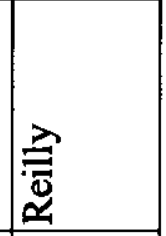 & \\
\hline & 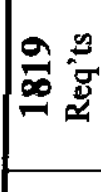 & $\begin{array}{l}r \\
\dot{m} \\
\infty \\
\dot{c} \\
i\end{array}$ & & 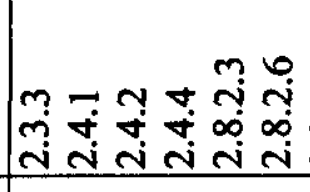 & 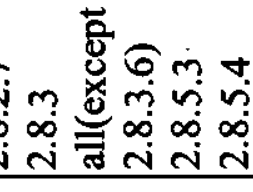 & בั & 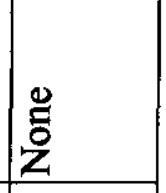 & \\
\hline & 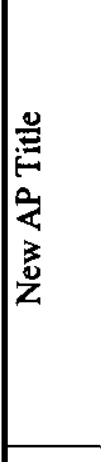 & 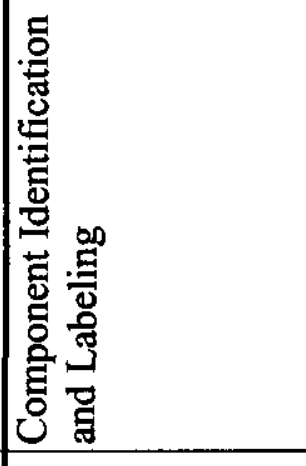 & & 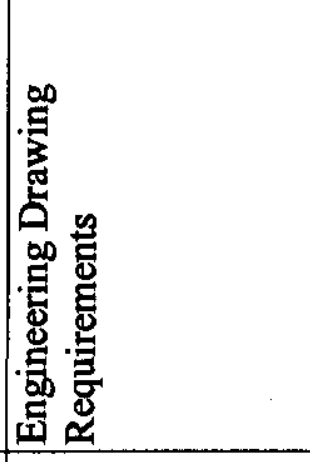 & & 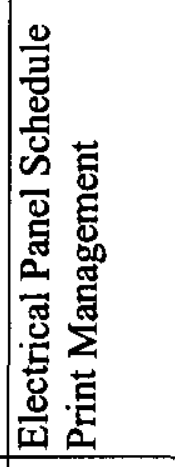 & 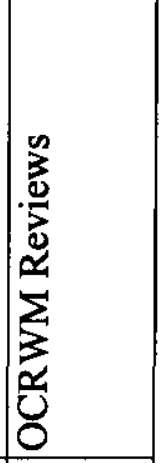 & \\
\hline & 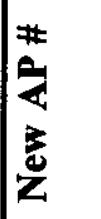 & $\mid \begin{array}{l}n \\
0 \\
0 \\
1 \\
1 \\
z\end{array}$ & & $\begin{array}{l}l \\
0 \\
1 \\
0 \\
1 \\
z \\
\text { I }\end{array}$ & & $\mid \begin{array}{l}\infty \\
0 \\
1 \\
0 \\
z \\
z \\
1\end{array}$ & $\mid \begin{array}{l}8 \\
0 \\
1 \\
1 \\
z \\
z\end{array}$ & \\
\hline
\end{tabular}




\begin{tabular}{|c|c|c|c|c|c|c|}
\hline \multirow{6}{*}{ 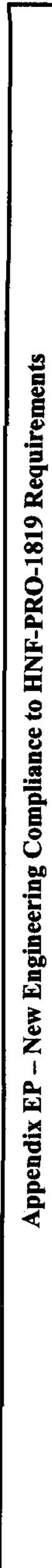 } & 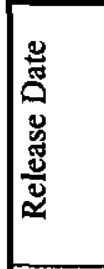 & 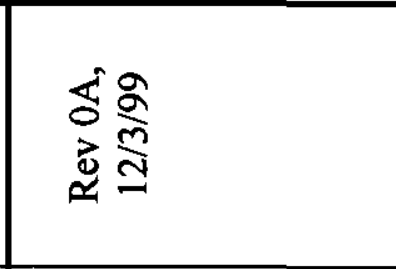 & 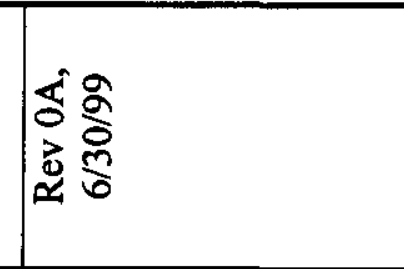 & 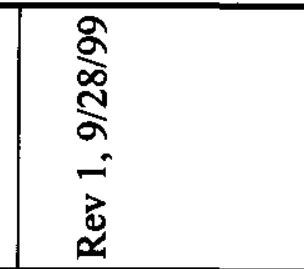 & 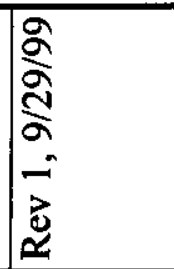 & 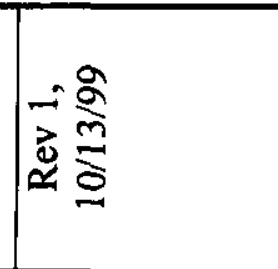 \\
\hline & 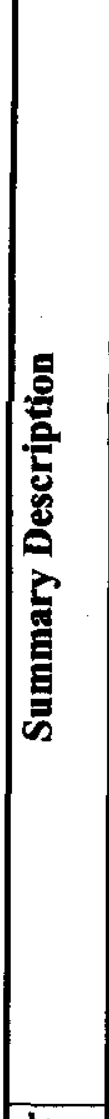 & 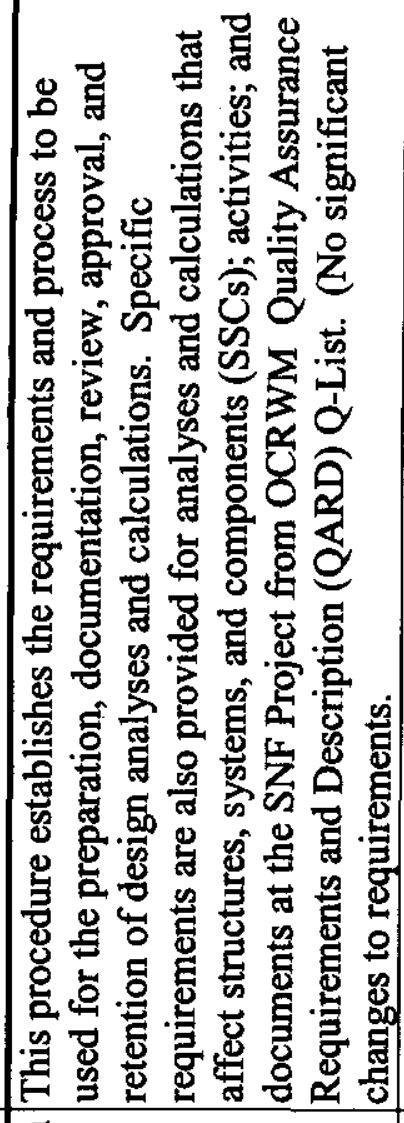 & 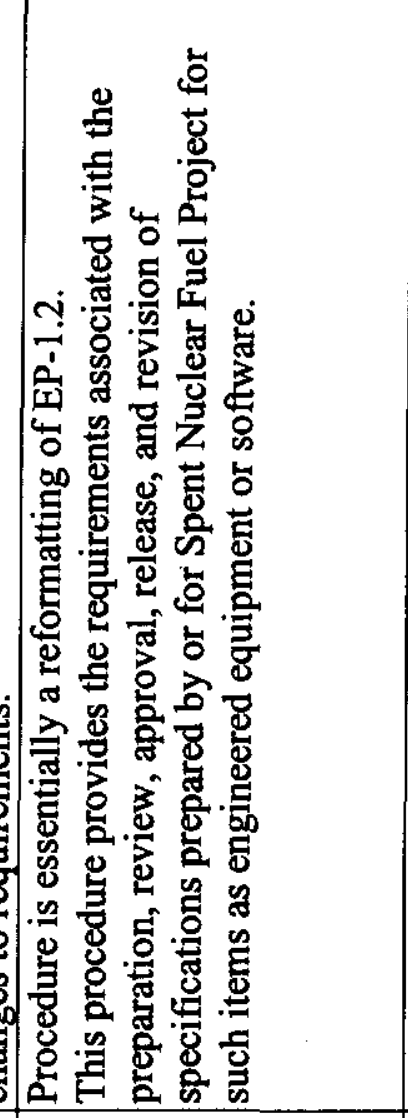 & 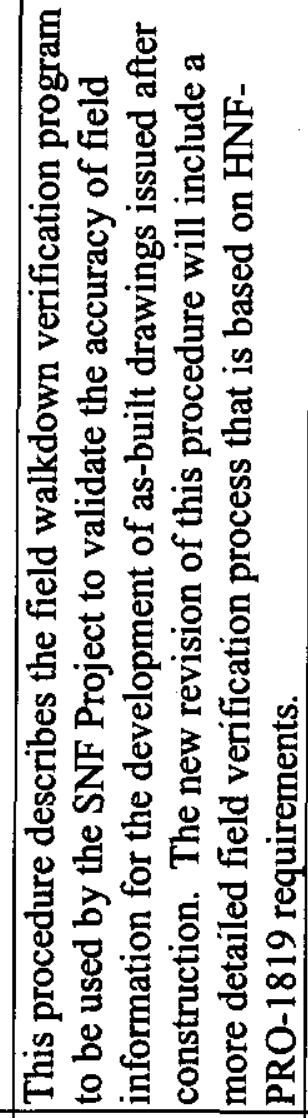 & 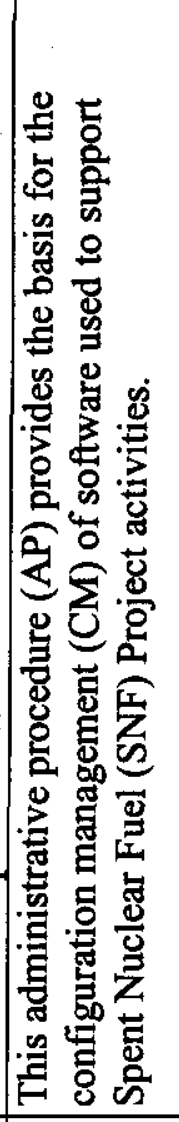 & 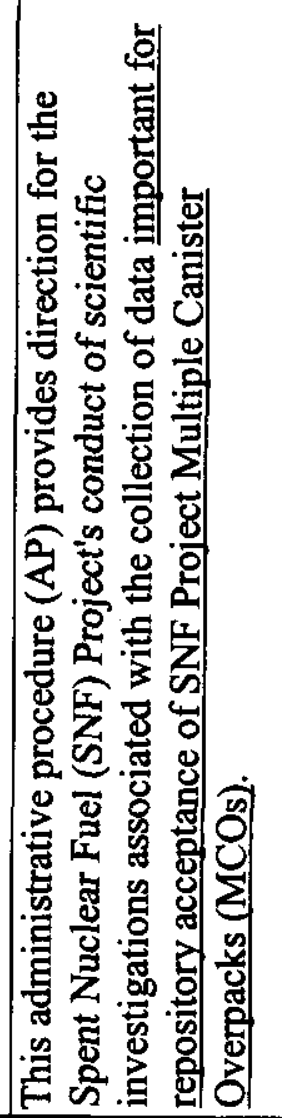 \\
\hline & 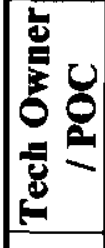 & 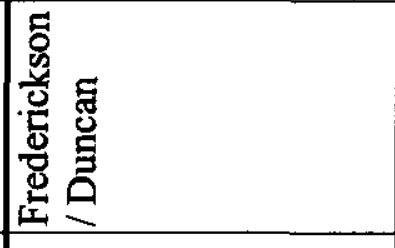 & 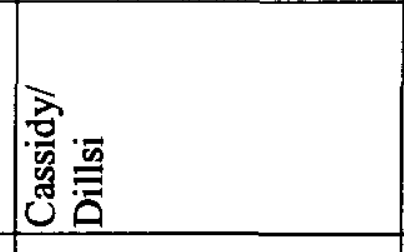 & 宽 & 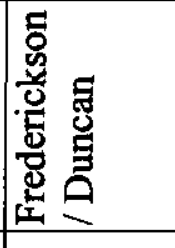 & 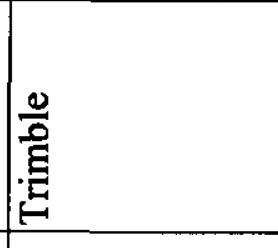 \\
\hline & 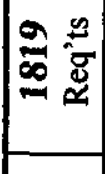 & 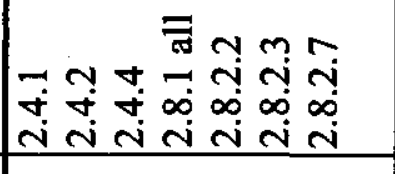 & 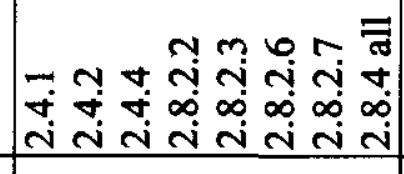 & 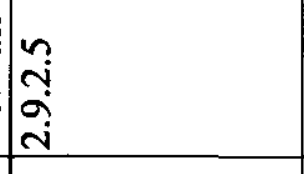 & \begin{tabular}{|l} 
\\
\\
\end{tabular} & 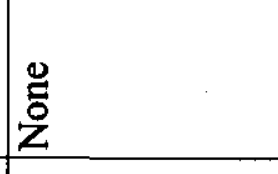 \\
\hline & 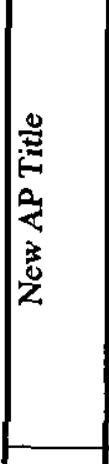 & 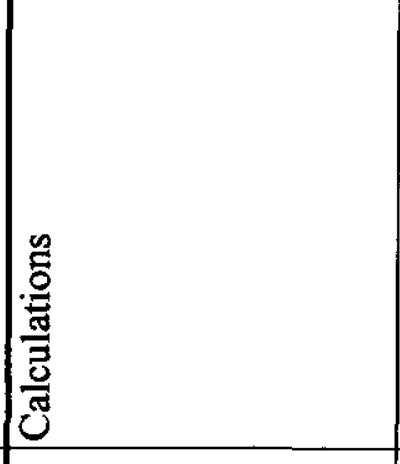 & 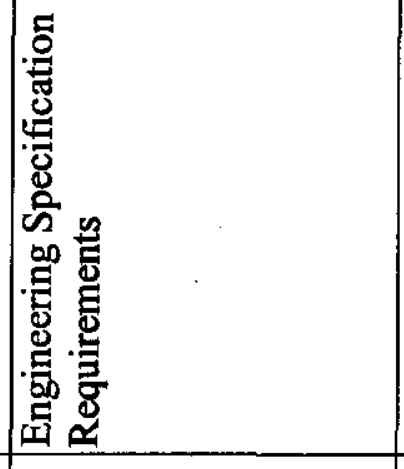 & 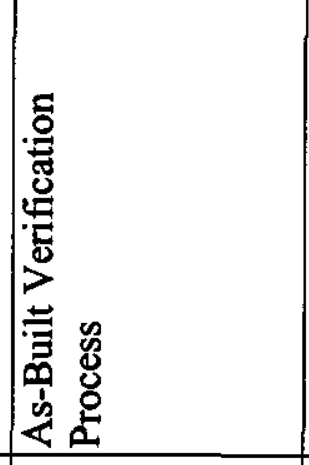 & 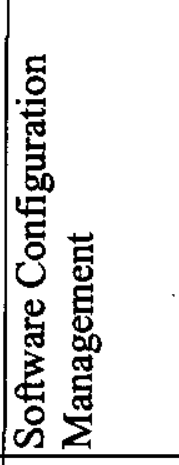 & 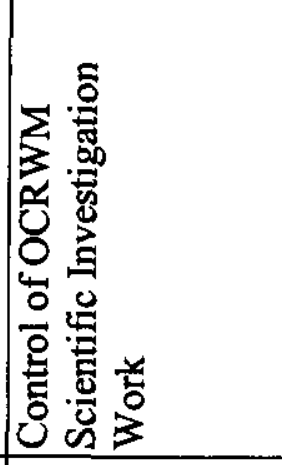 \\
\hline & z & 옹 & $\begin{array}{l}= \\
0 \\
0 \\
b \\
i \\
i \\
1\end{array}$ & $\mid \begin{array}{c}N \\
0 \\
0 \\
1 \\
⿱ 亠 乂 \\
1\end{array}$ & $\begin{array}{l}m \\
0 \\
0 \\
b \\
1 \\
j \\
0\end{array}$ & $\mid \begin{array}{l}n \\
0 \\
0 \\
1 \\
1 \\
1 \\
1\end{array}$ \\
\hline
\end{tabular}




\begin{tabular}{|c|c|c|c|c|c|c|c|}
\hline \multirow{6}{*}{ |c } & 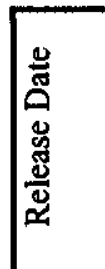 & $\underset{\substack{a \\
m}}{\stackrel{a}{a}}$ & 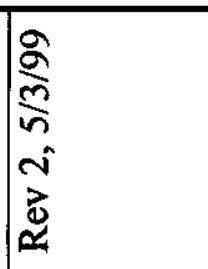 & 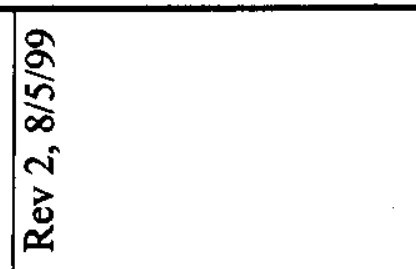 & 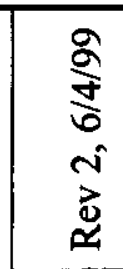 & 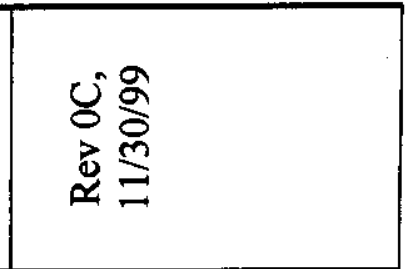 & \\
\hline & 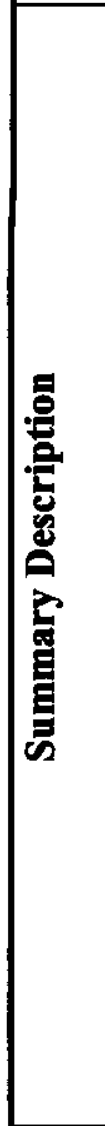 & 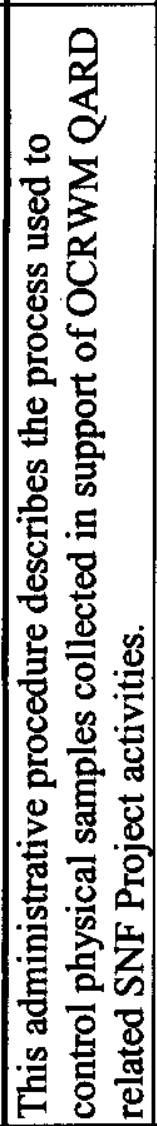 & 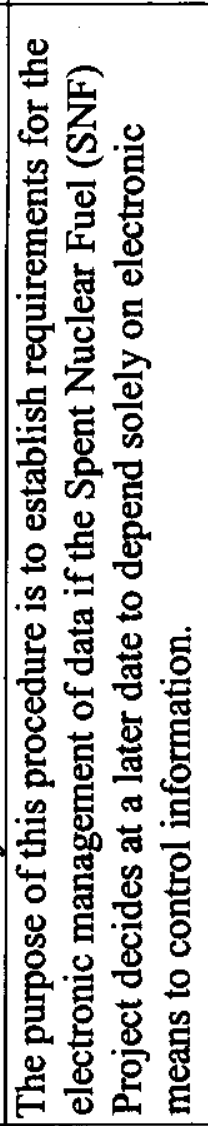 & 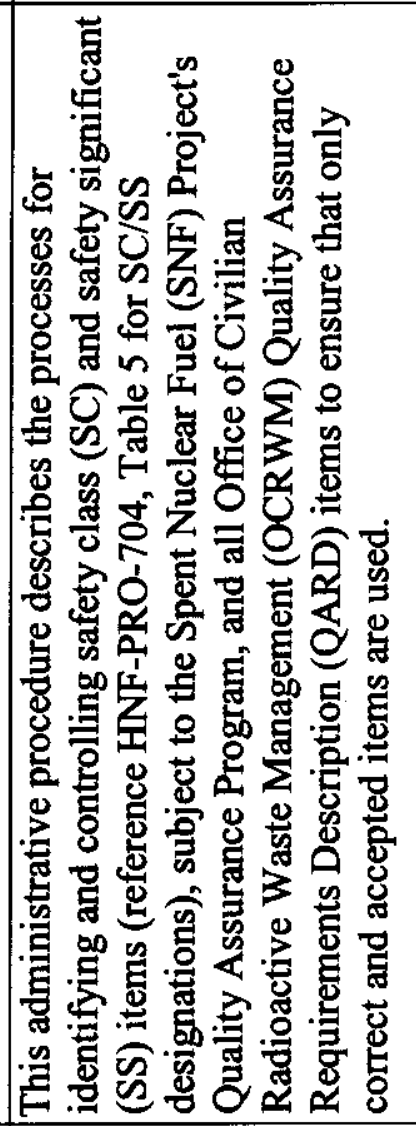 & 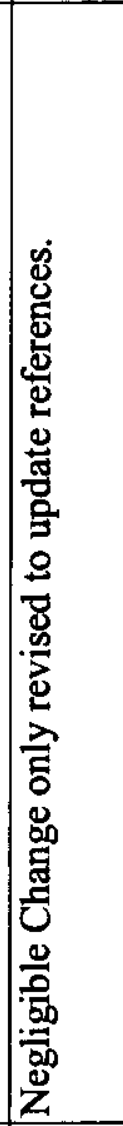 & 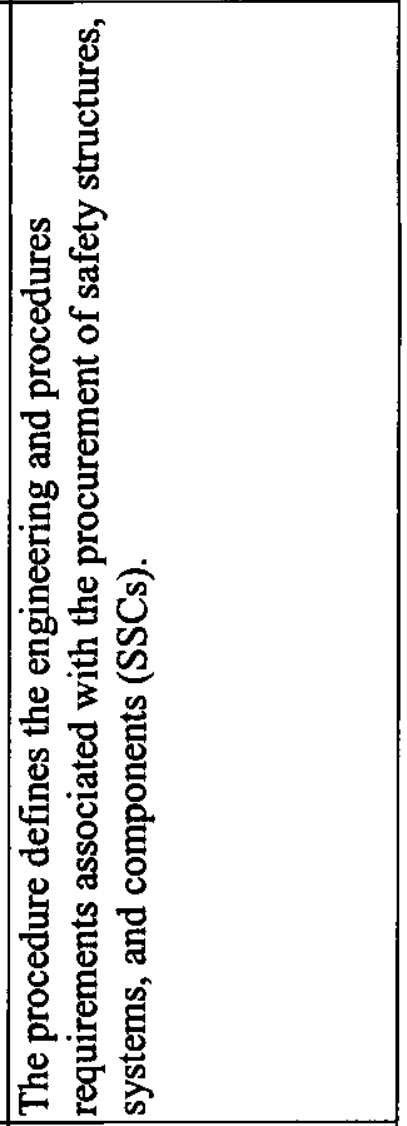 & $\begin{array}{l}\text { 离 } \\
\end{array}$ \\
\hline & 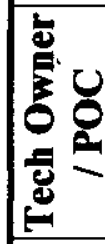 & 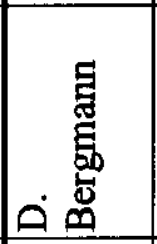 & 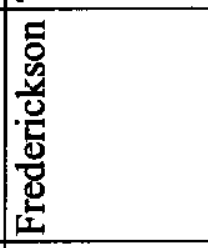 & 总 & 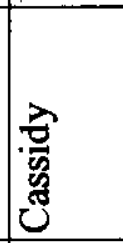 & 总票 & \\
\hline & $\frac{a}{\infty} \stackrel{0}{\square}$ & : & Z & 己 & Z & 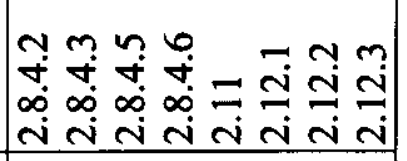 & \\
\hline & 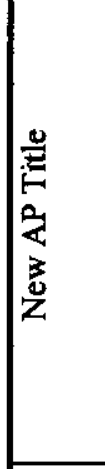 & 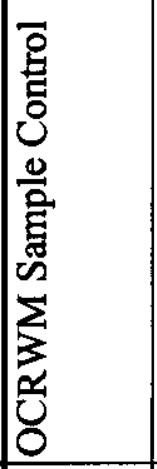 & 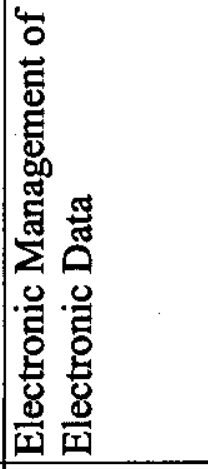 & 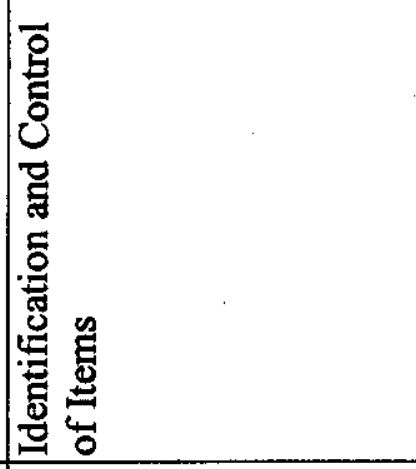 & 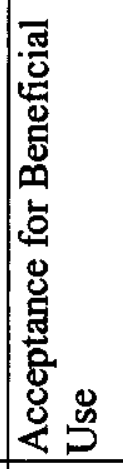 & 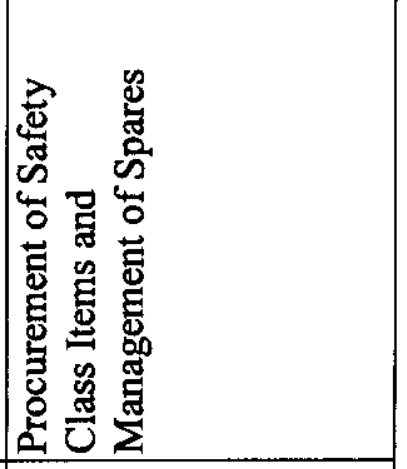 & \\
\hline & 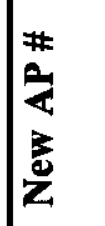 & $\mid \begin{array}{l}0 \\
0 \\
0 \\
z \\
z\end{array}$ & $\begin{array}{l}n \\
0 \\
0 \\
0 \\
\dot{z} \\
1\end{array}$ & $\mid \begin{array}{l}\infty \\
0 \\
1 \\
b \\
\vdots \\
\text { ż }\end{array}$ & $\begin{array}{l}9 \\
0 \\
1 \\
1 \\
1 \\
0\end{array}$ & $\mid \begin{array}{l}0 \\
0 \\
1 \\
b \\
1 \\
z \\
1\end{array}$ & \\
\hline
\end{tabular}




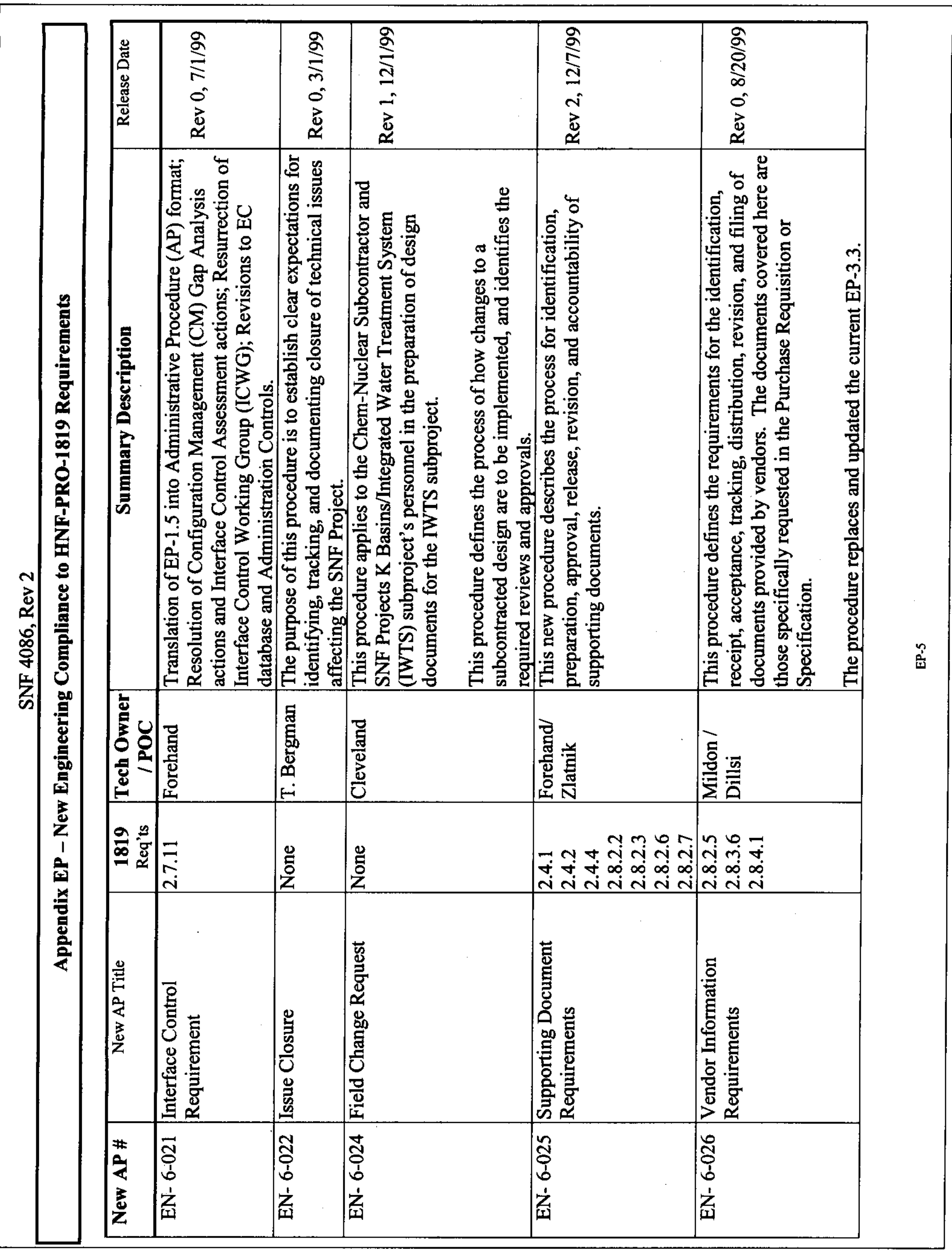




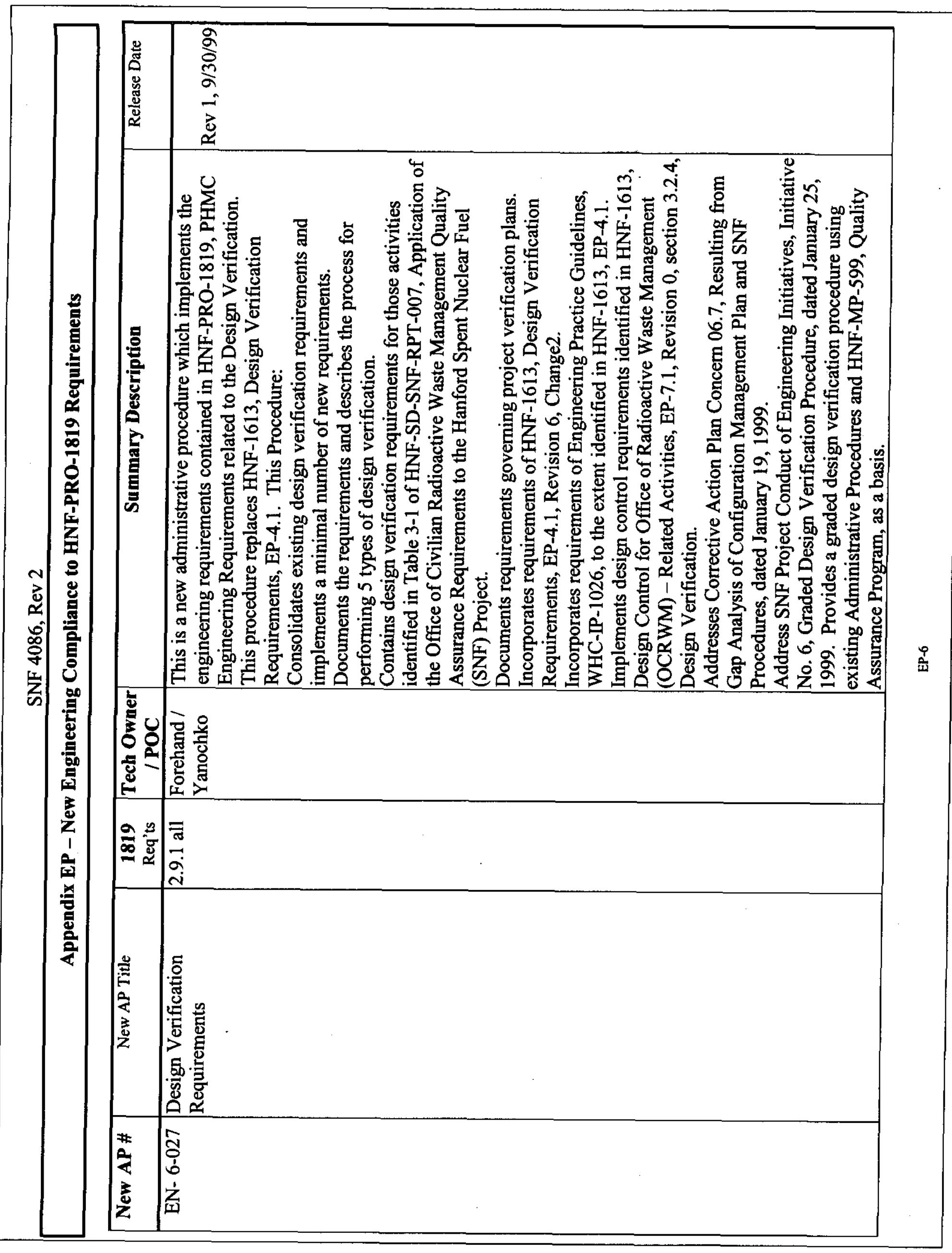




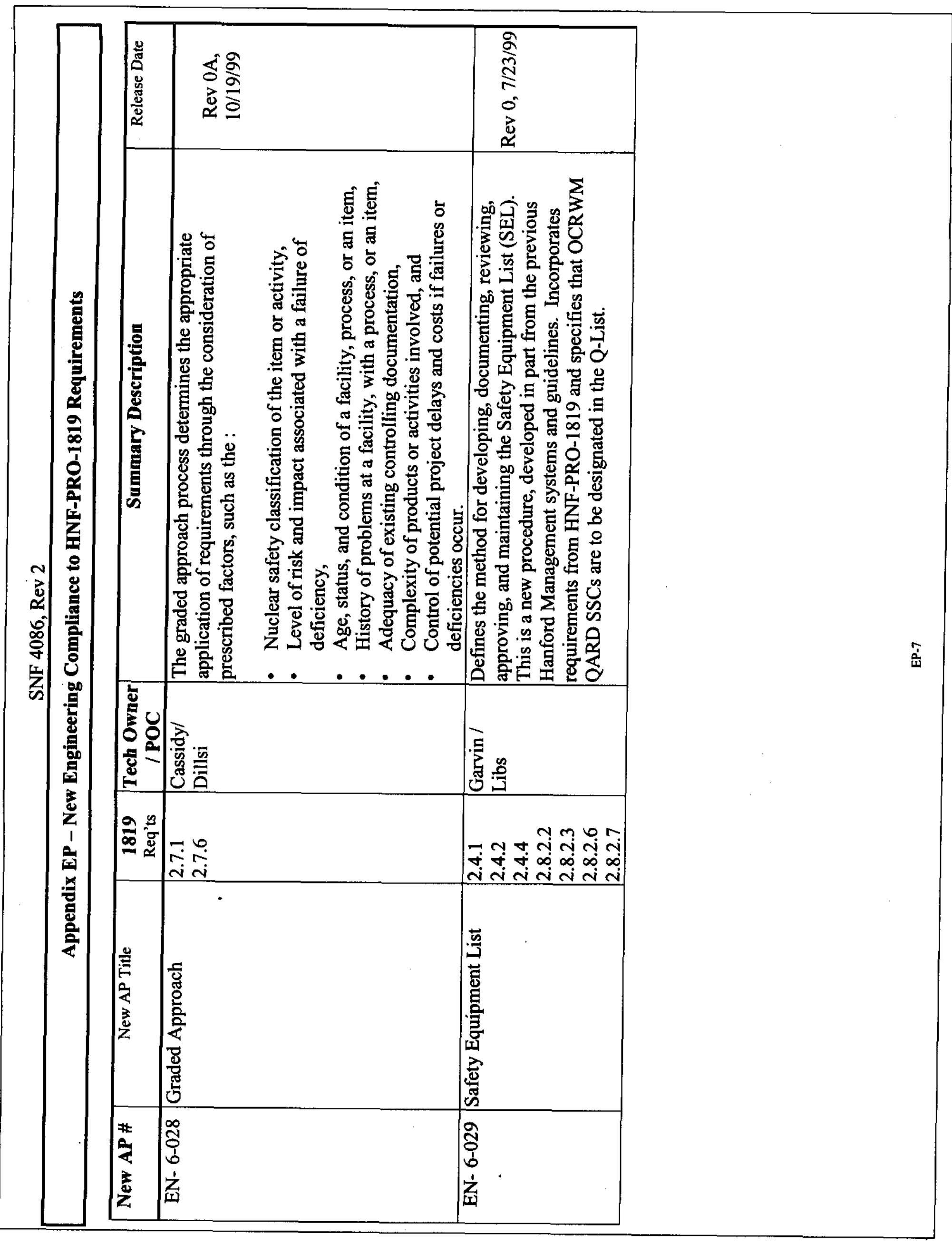




\begin{tabular}{|c|c|c|c|c|c|c|c|}
\hline & 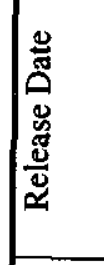 & 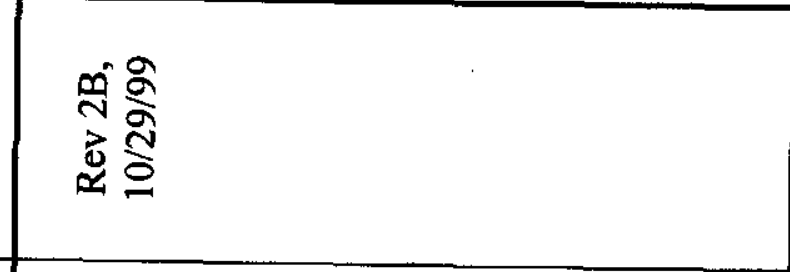 & 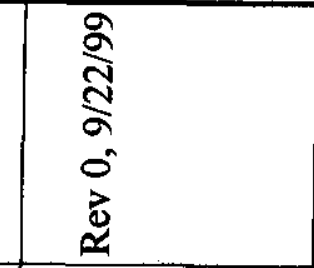 & & 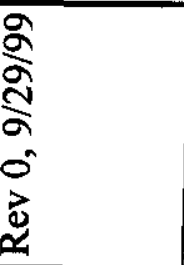 & 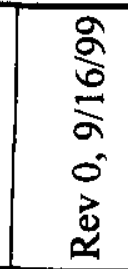 & 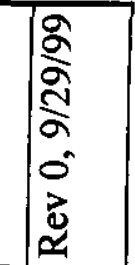 \\
\hline 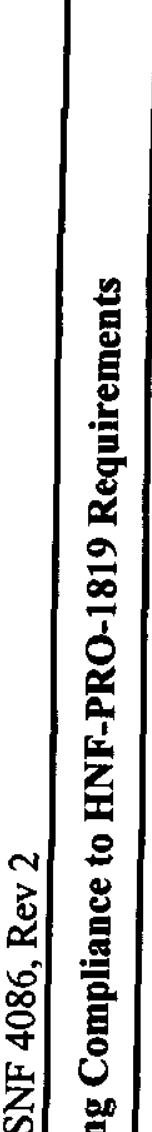 & 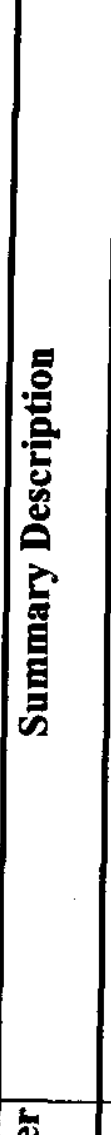 & 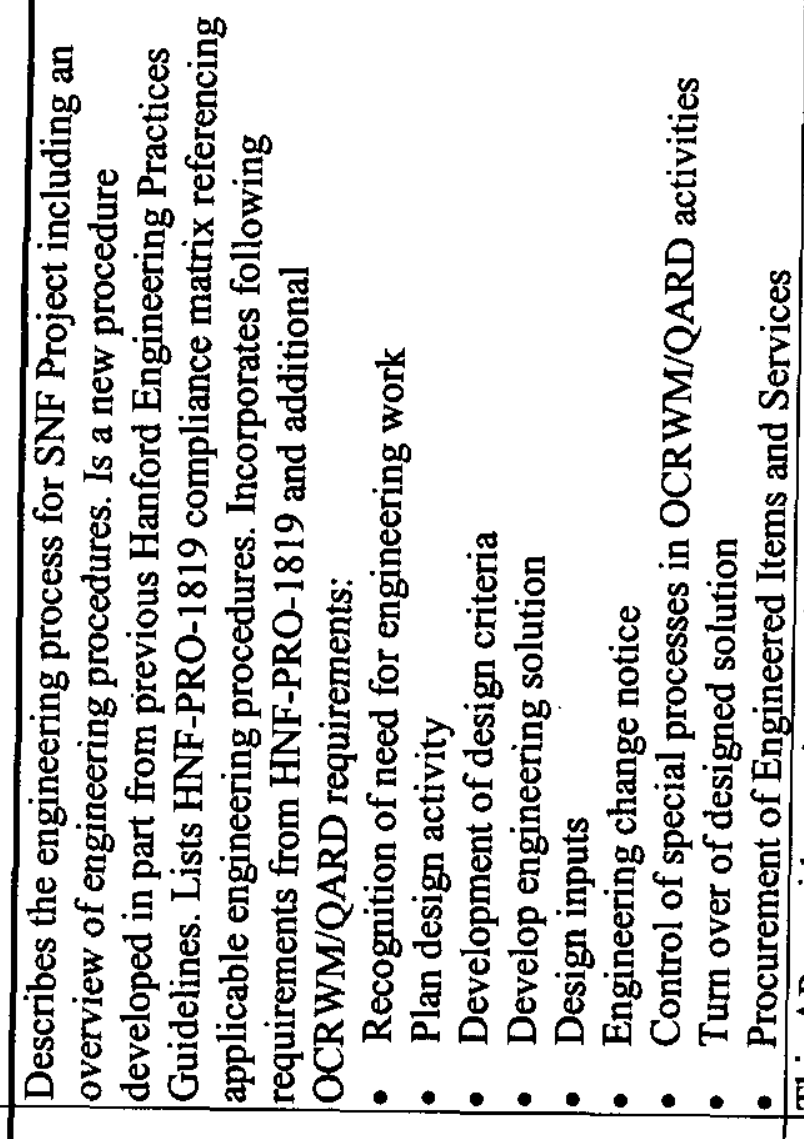 & 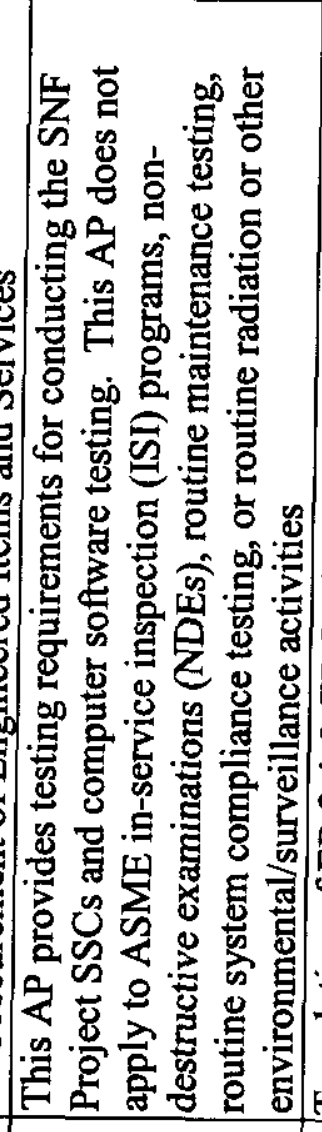 & 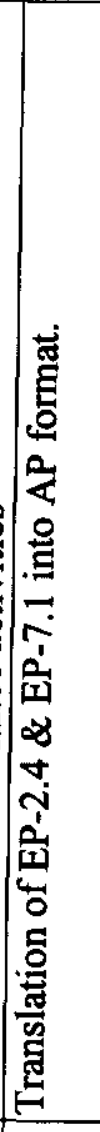 & 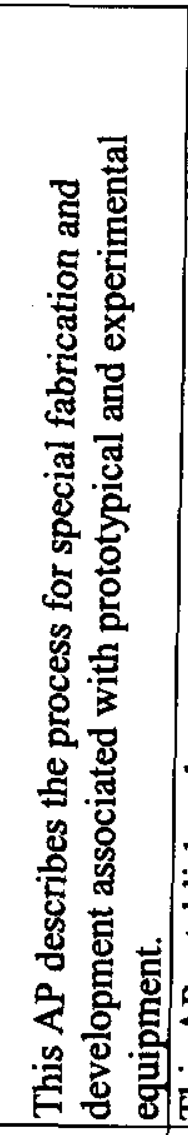 & 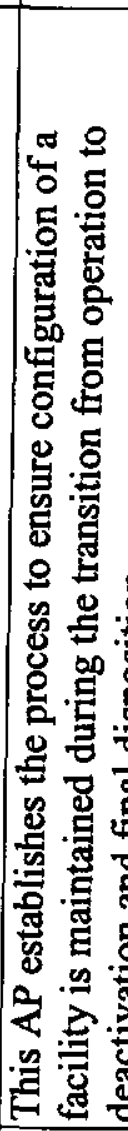 & 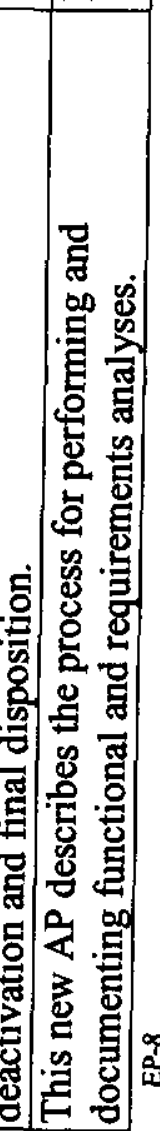 \\
\hline 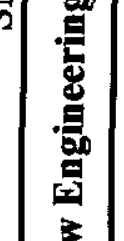 & 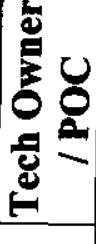 & 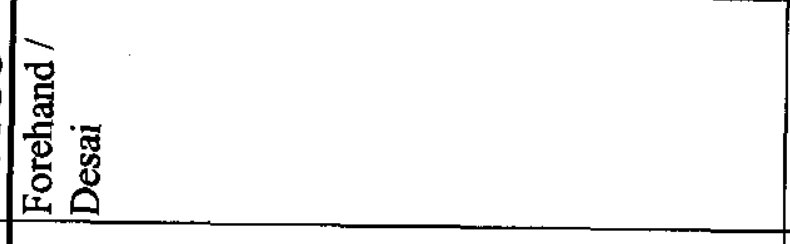 & 宸 & 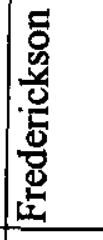 & & 冬 & 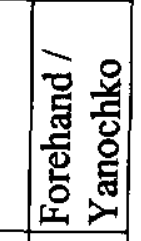 \\
\hline 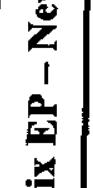 & a) & 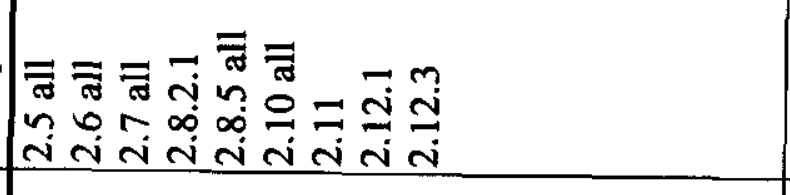 & 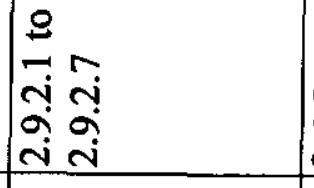 & $\begin{array}{l}n \\
0 \\
0 \\
0\end{array}$ & & 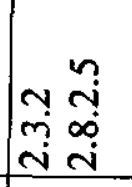 & \begin{tabular}{|l} 
号 \\
Z \\
\end{tabular} \\
\hline$\frac{2}{4}$ & 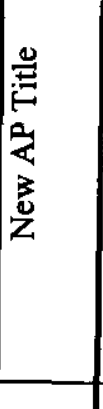 & 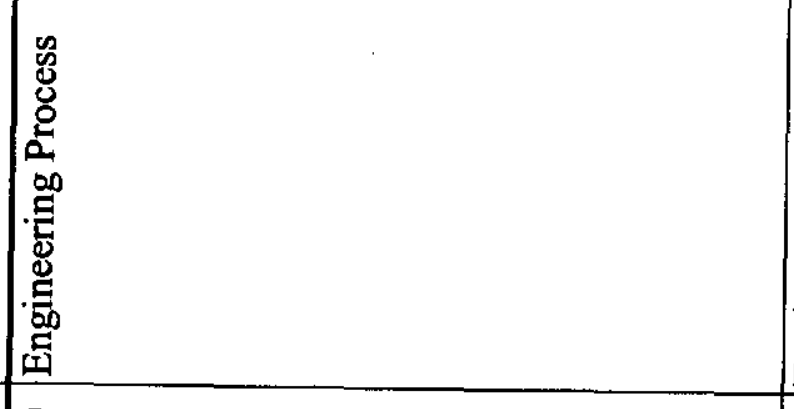 & 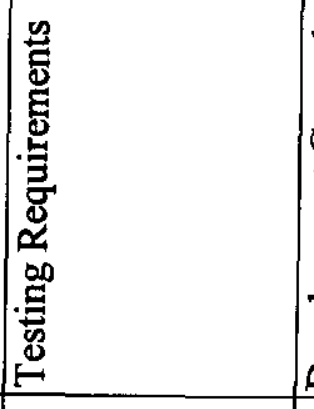 & 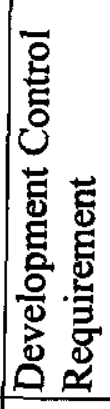 & & 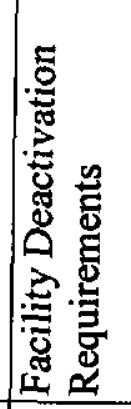 & 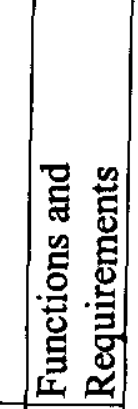 \\
\hline & $\frac{1}{2}$ & 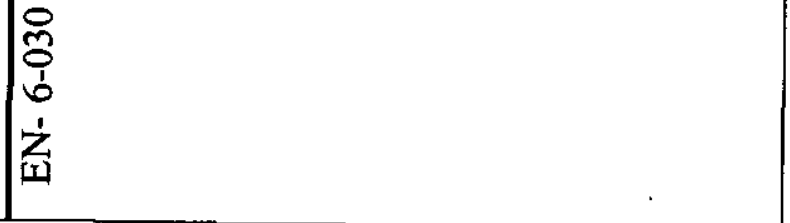 & $\begin{array}{l}\bar{m} \\
0 \\
b \\
\dot{1} \\
\dot{1}\end{array}$ & 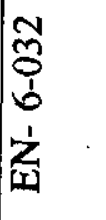 & & $\begin{array}{l}m \\
0 \\
0 \\
0 \\
\dot{z} \\
\dot{z}\end{array}$ & $\mid \begin{array}{c}0 \\
0 \\
0 \\
0 \\
\dot{1} \\
\dot{y}\end{array}$ \\
\hline
\end{tabular}




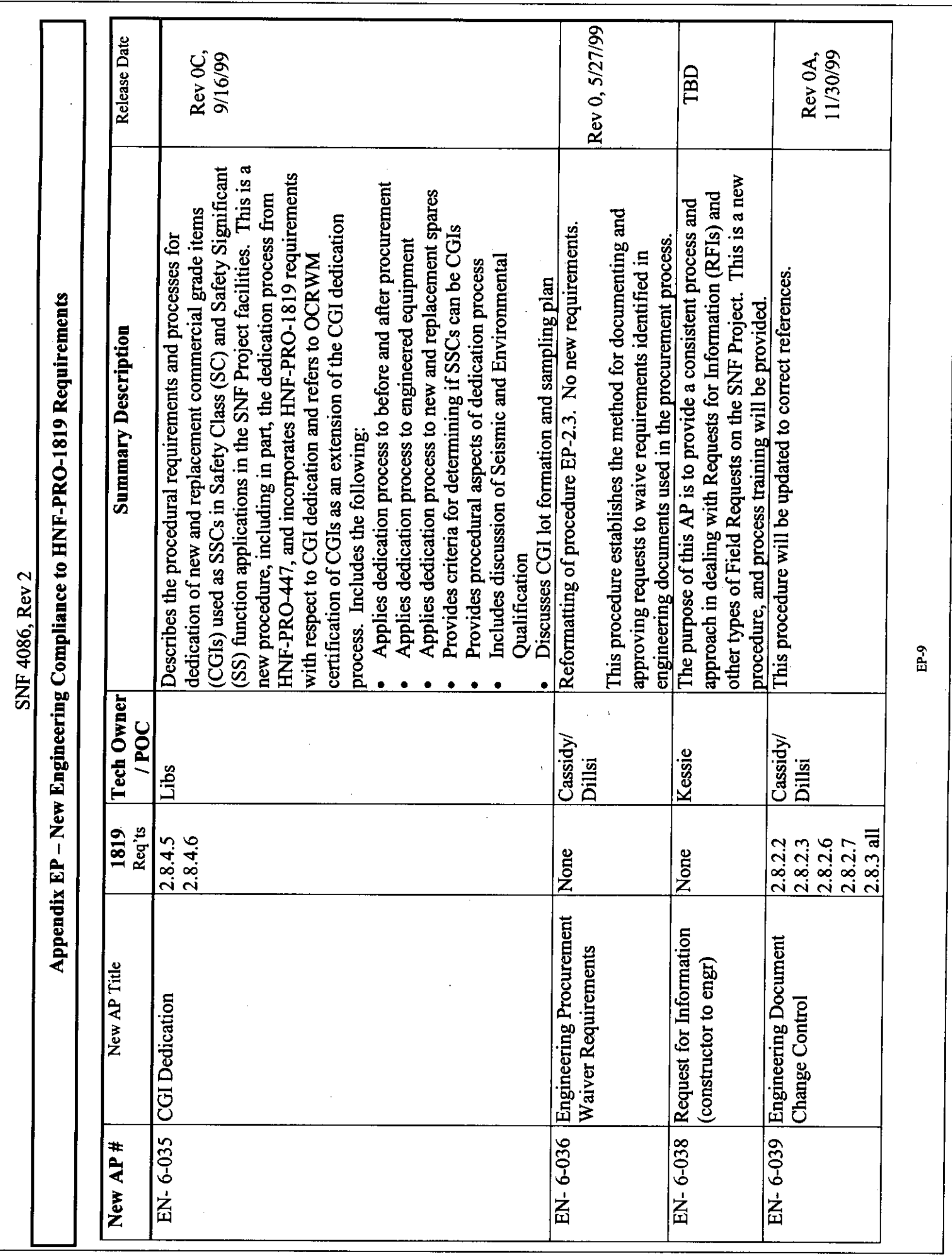




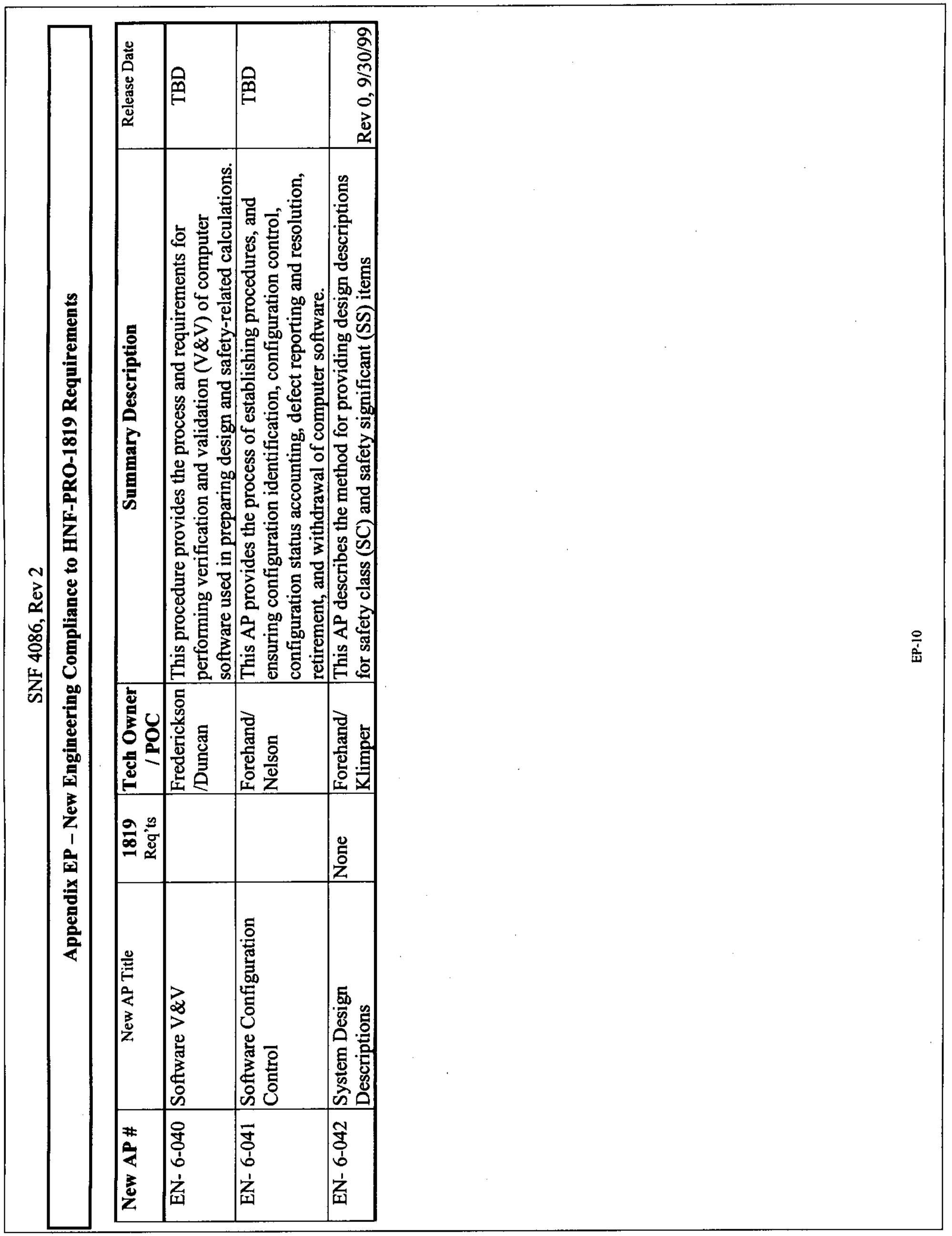




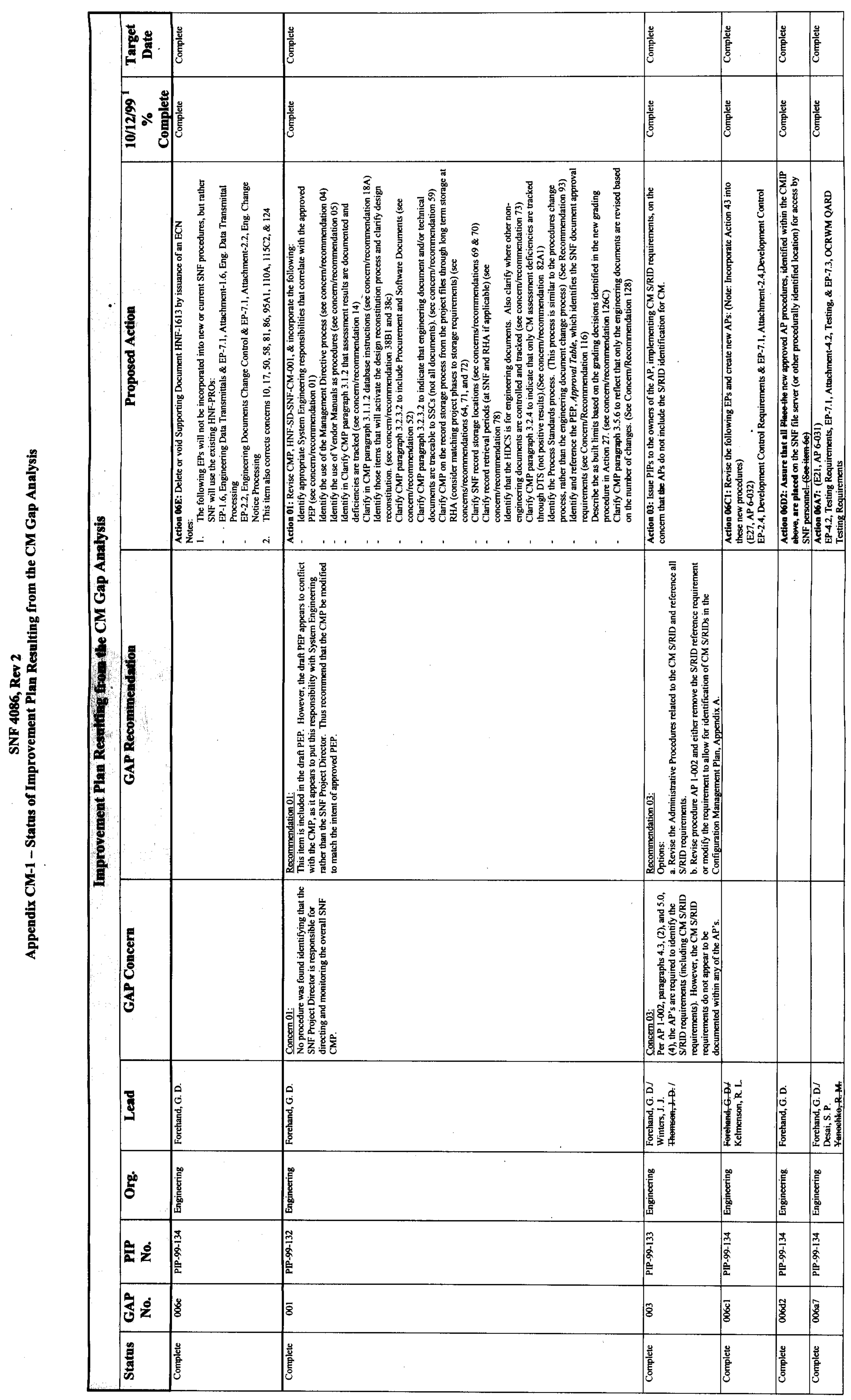




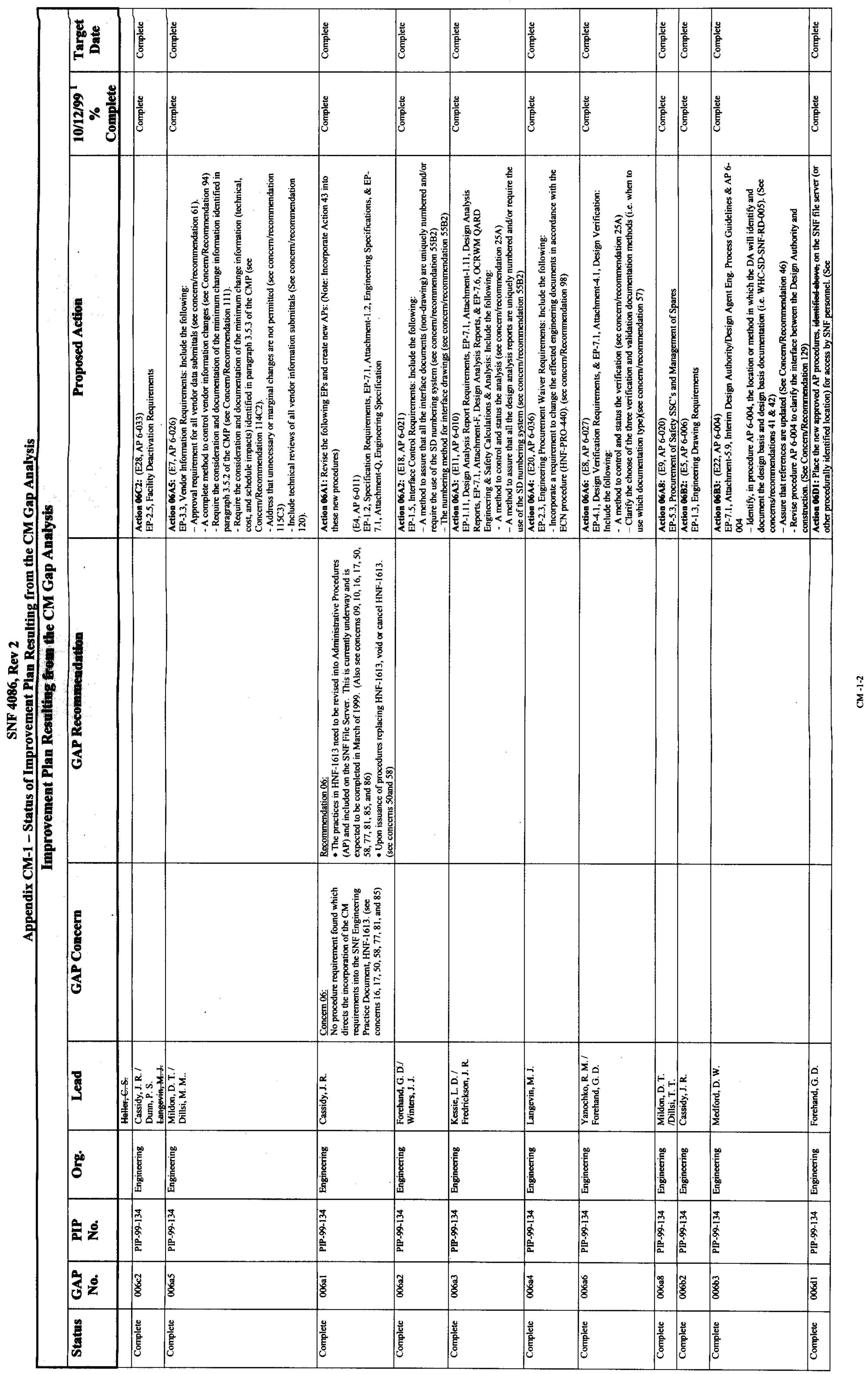




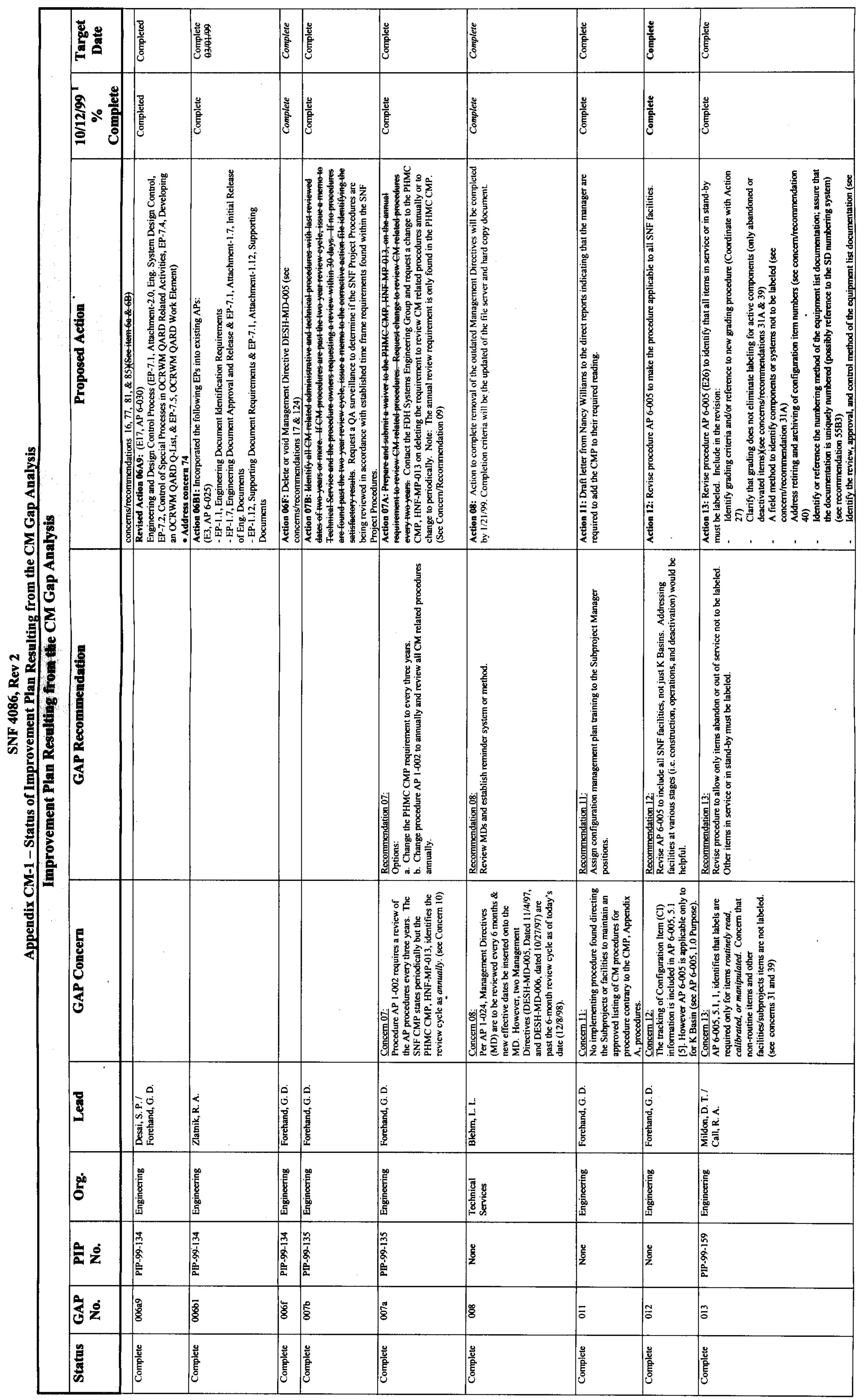




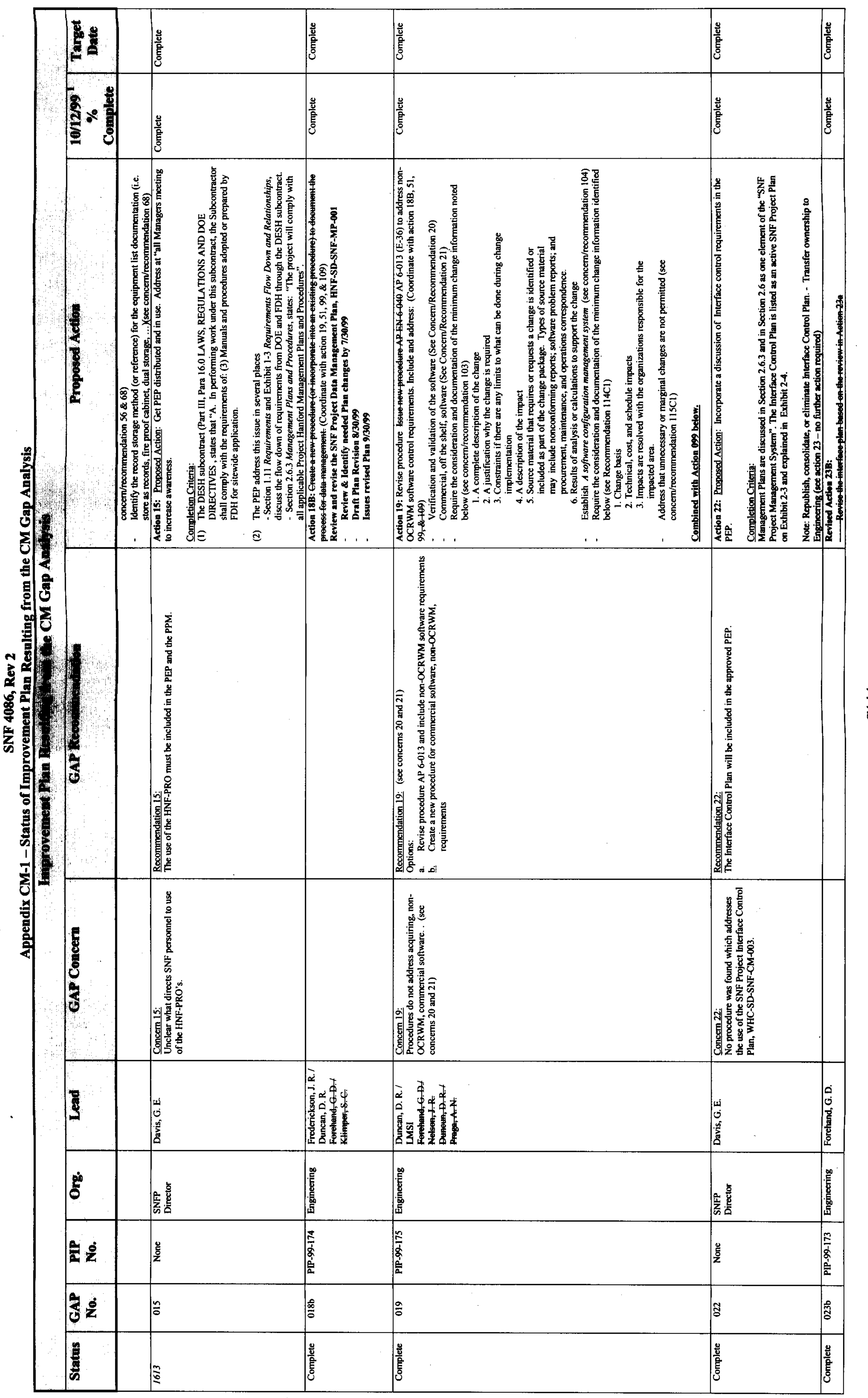




\begin{tabular}{|c|c|c|c|c|c|c|c|c|}
\hline 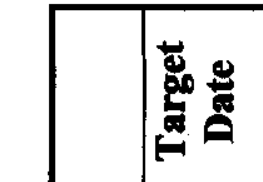 & 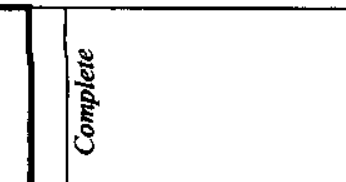 & 总 & 善 & 竞 & 黨 & 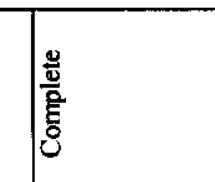 & 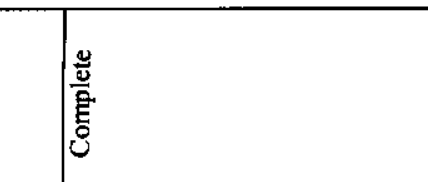 & $\frac{\mathscr{z}}{\underline{z}}$ \\
\hline 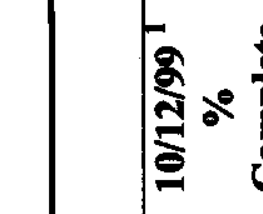 & 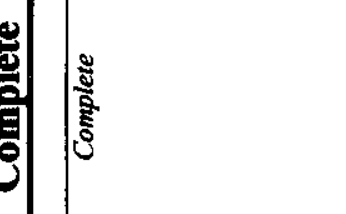 & 量 & 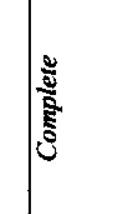 & 童 & 总 & $\mid \frac{\underline{z}}{5}$ & 善 & 童 \\
\hline 毫 & 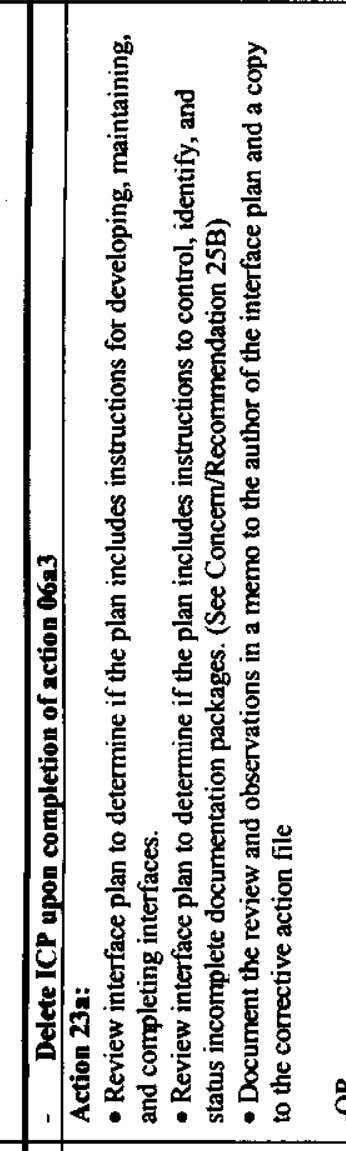 & 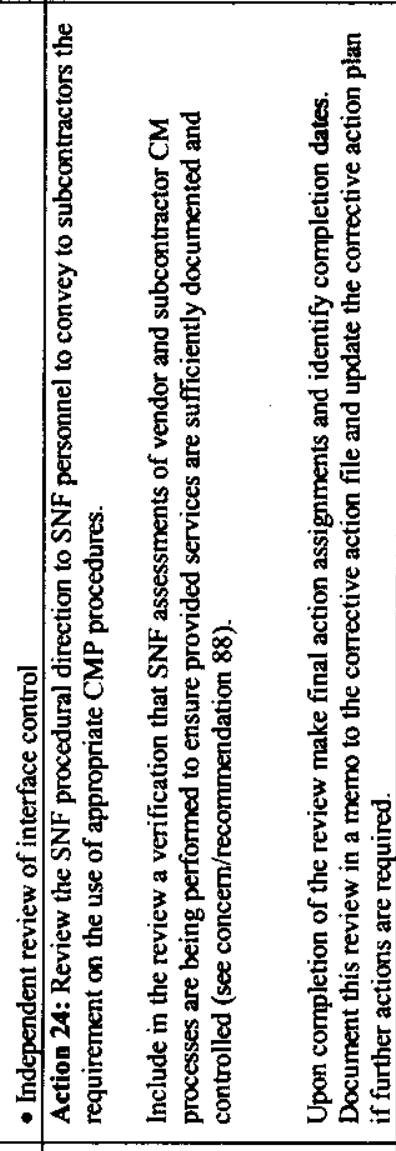 & 嫼 & 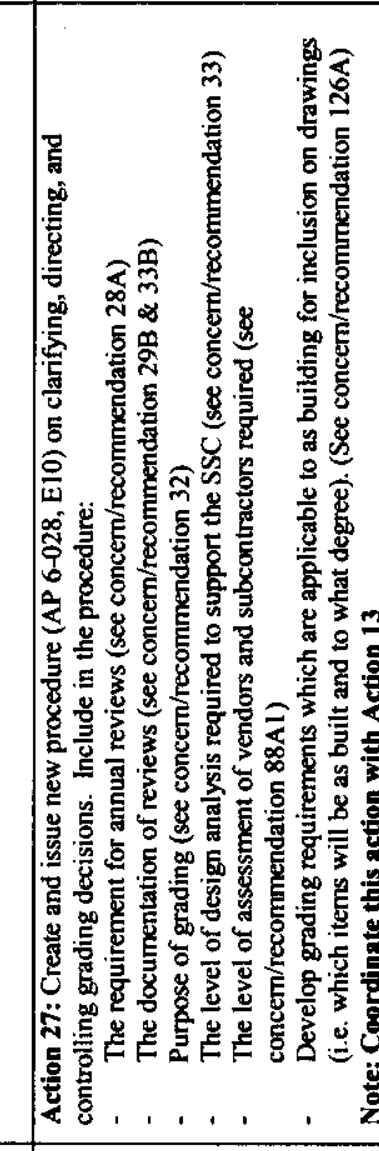 & 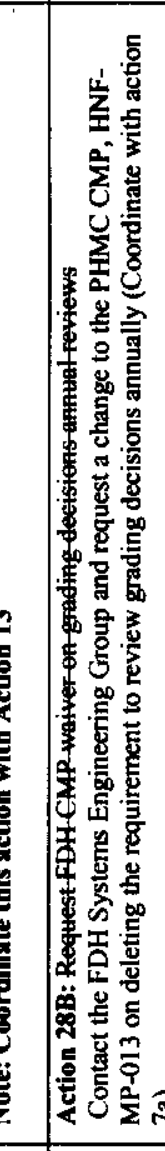 & 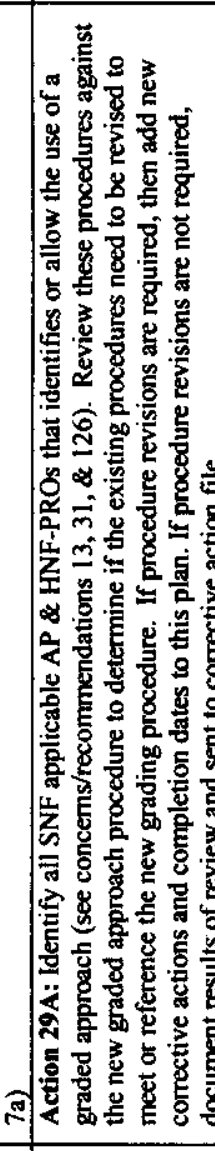 & 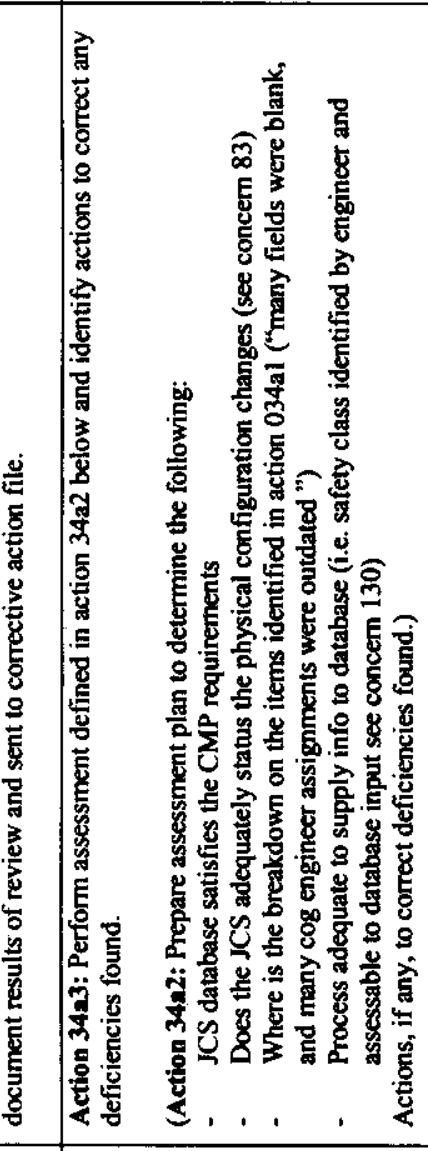 & 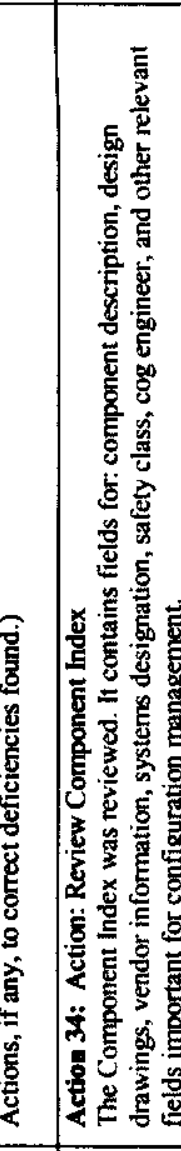 \\
\hline 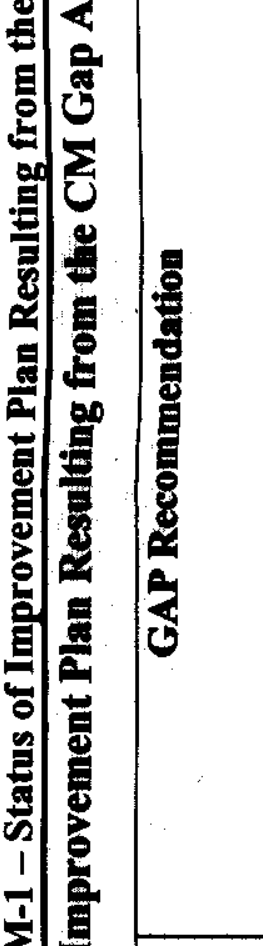 & 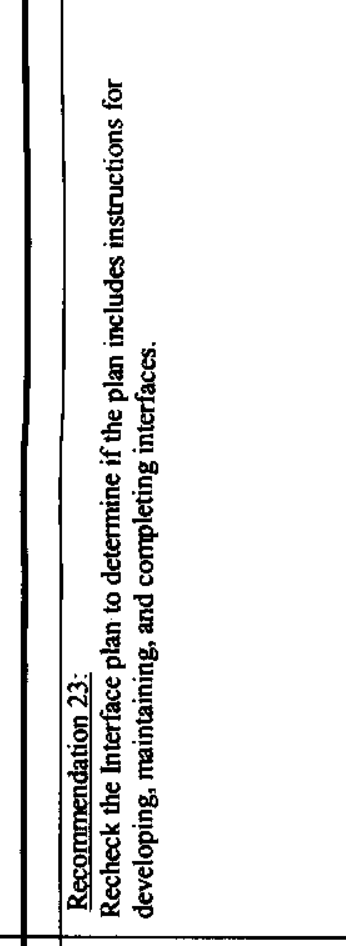 & 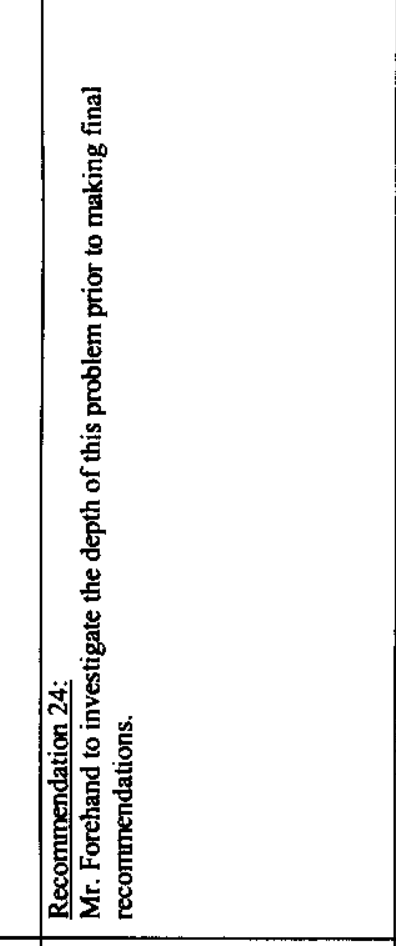 & 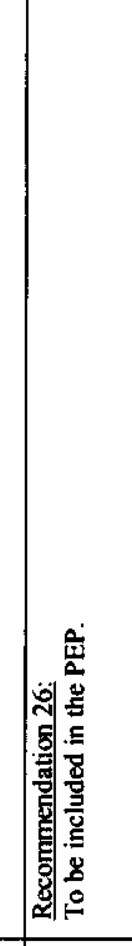 & 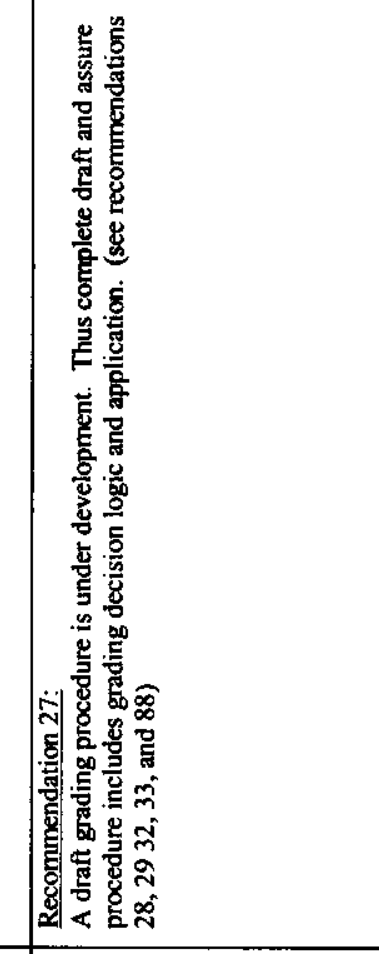 & & 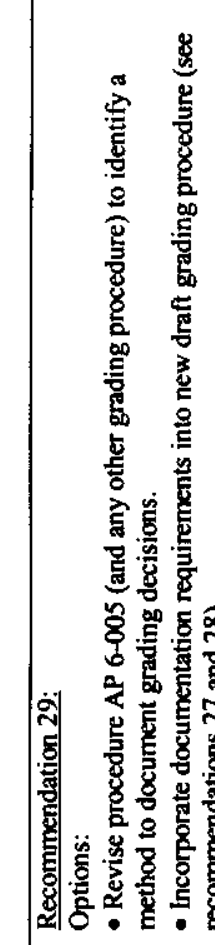 & & 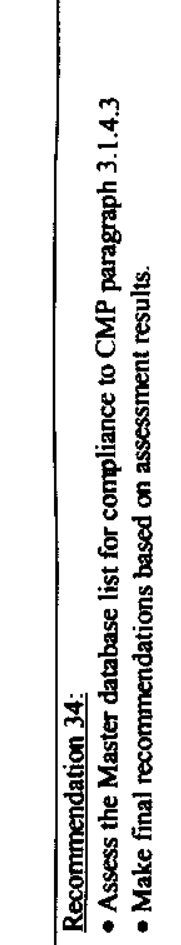 \\
\hline 怤 & 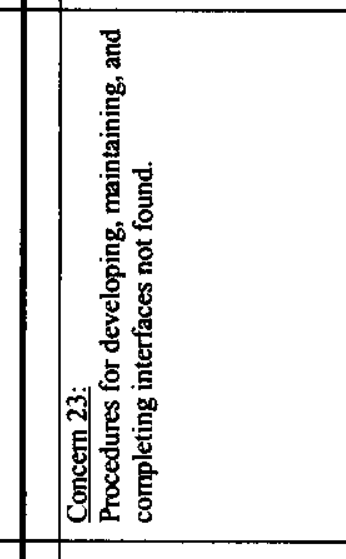 & 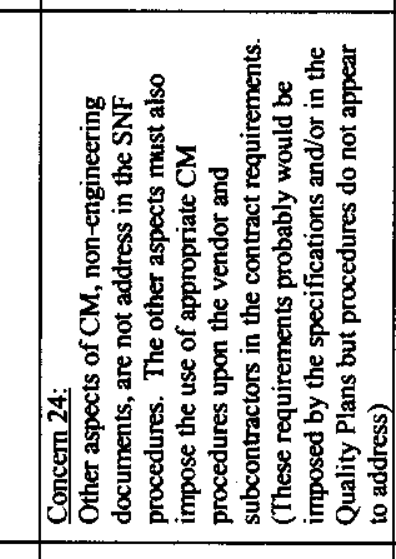 & 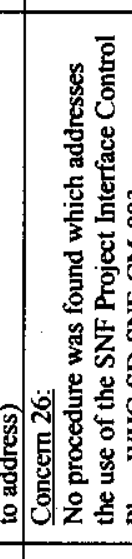 & 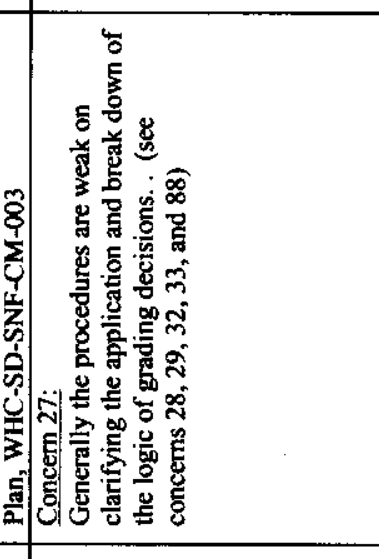 & & 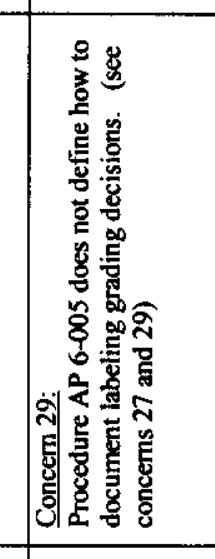 & & 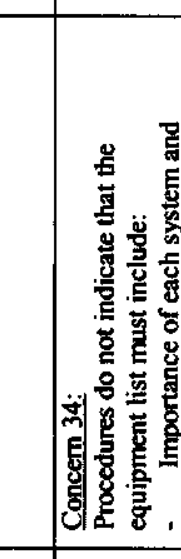 \\
\hline 递 & 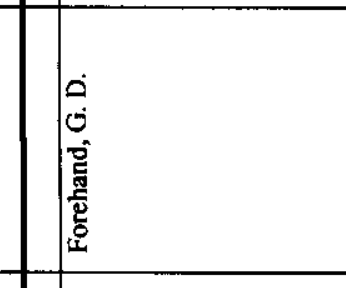 & 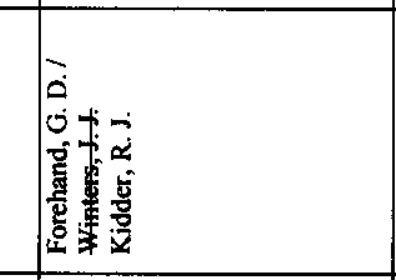 & 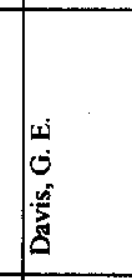 & 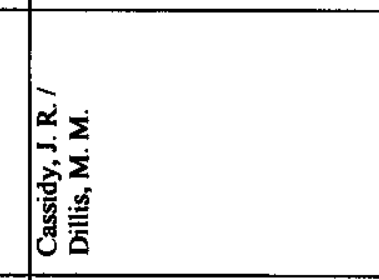 & 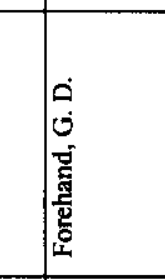 & 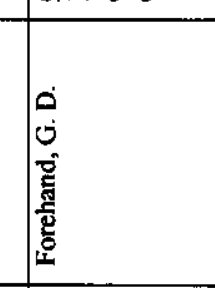 & 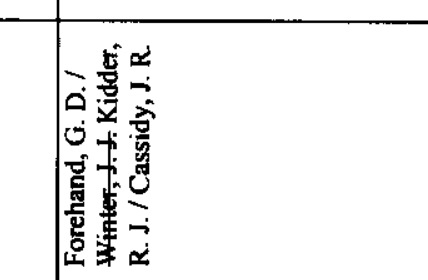 & 憙 \\
\hline हैं & $\mid$ & & 喜毫 & & |⿱\zh18灬心 & 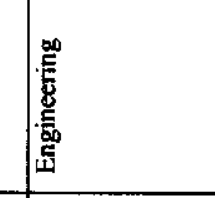 & $\mid$ & 噟 \\
\hline$\frac{1}{2}$ & 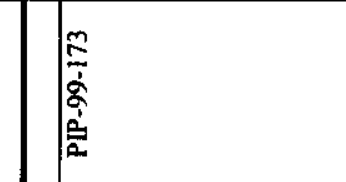 & 䇾 & $\frac{\mathrm{g}}{2}$ & 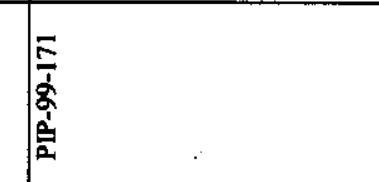 & $\frac{8}{\overbrace{2}^{2}}$ & $\frac{0}{2}$ & $\frac{2}{2}$ & $\frac{2}{2}$ \\
\hline 羿 & $\frac{\pi}{8}$ & $\stackrel{A}{8}$ & : & $\tilde{8}$ & 素 & : & 语 & 居 \\
\hline$\frac{\frac{g}{2}}{m}$ & 童 & $\mid \frac{\mathrm{s}}{\mathrm{n}}$ & 意 & $\mid \frac{\frac{2}{2}}{3}$ & 总 & $\mid \frac{2}{n_{3}}$ & 产 & 善 \\
\hline
\end{tabular}




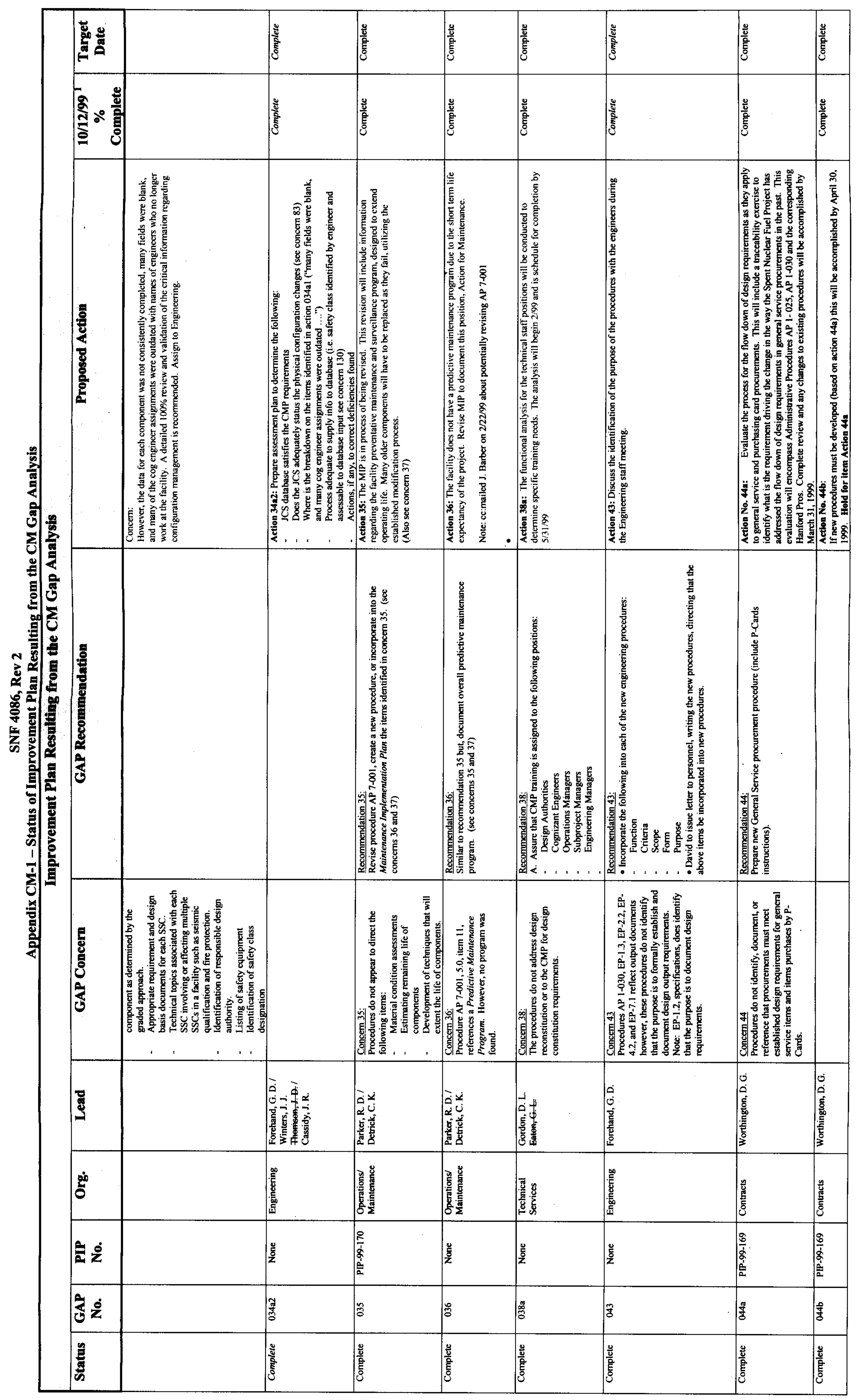




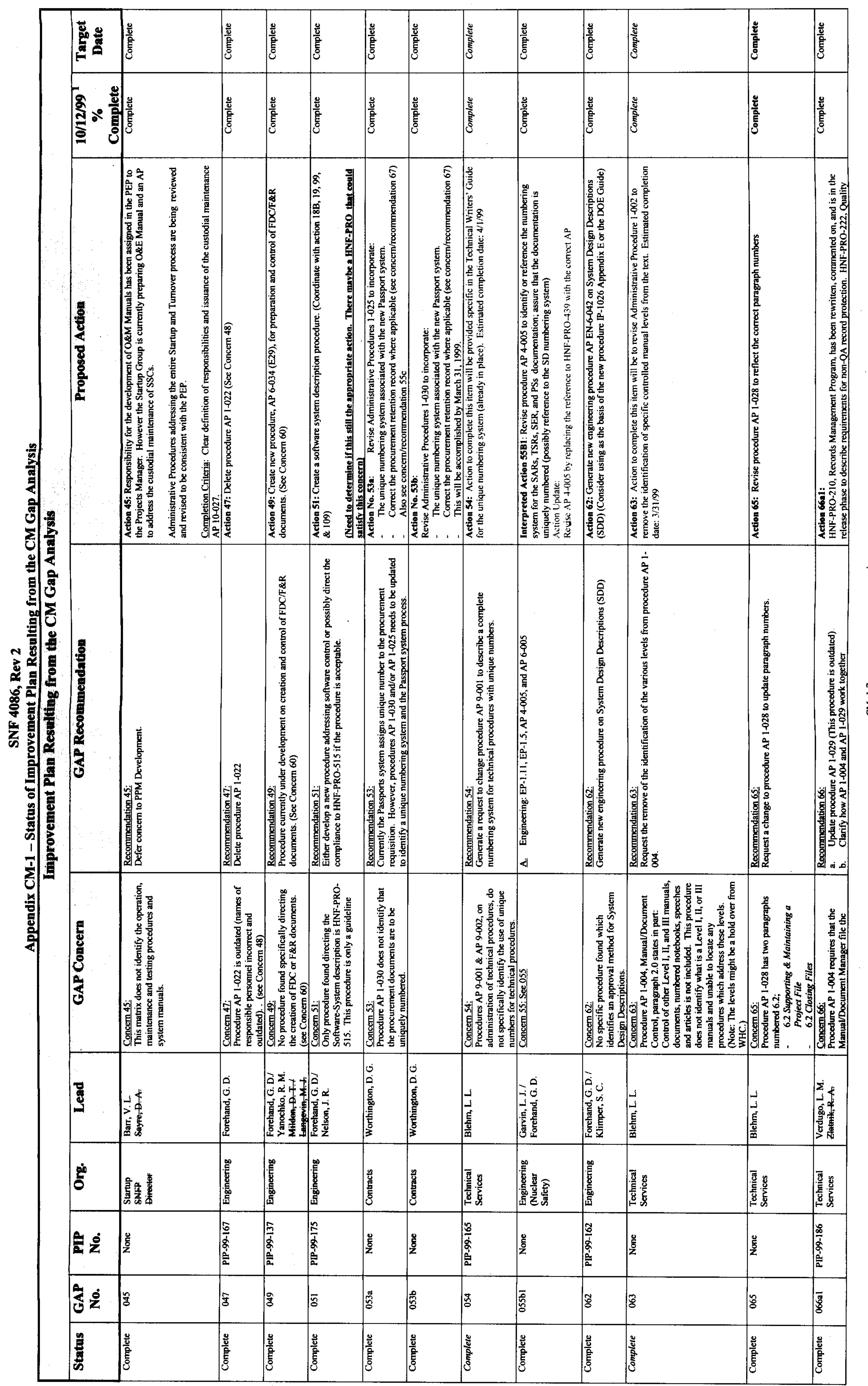




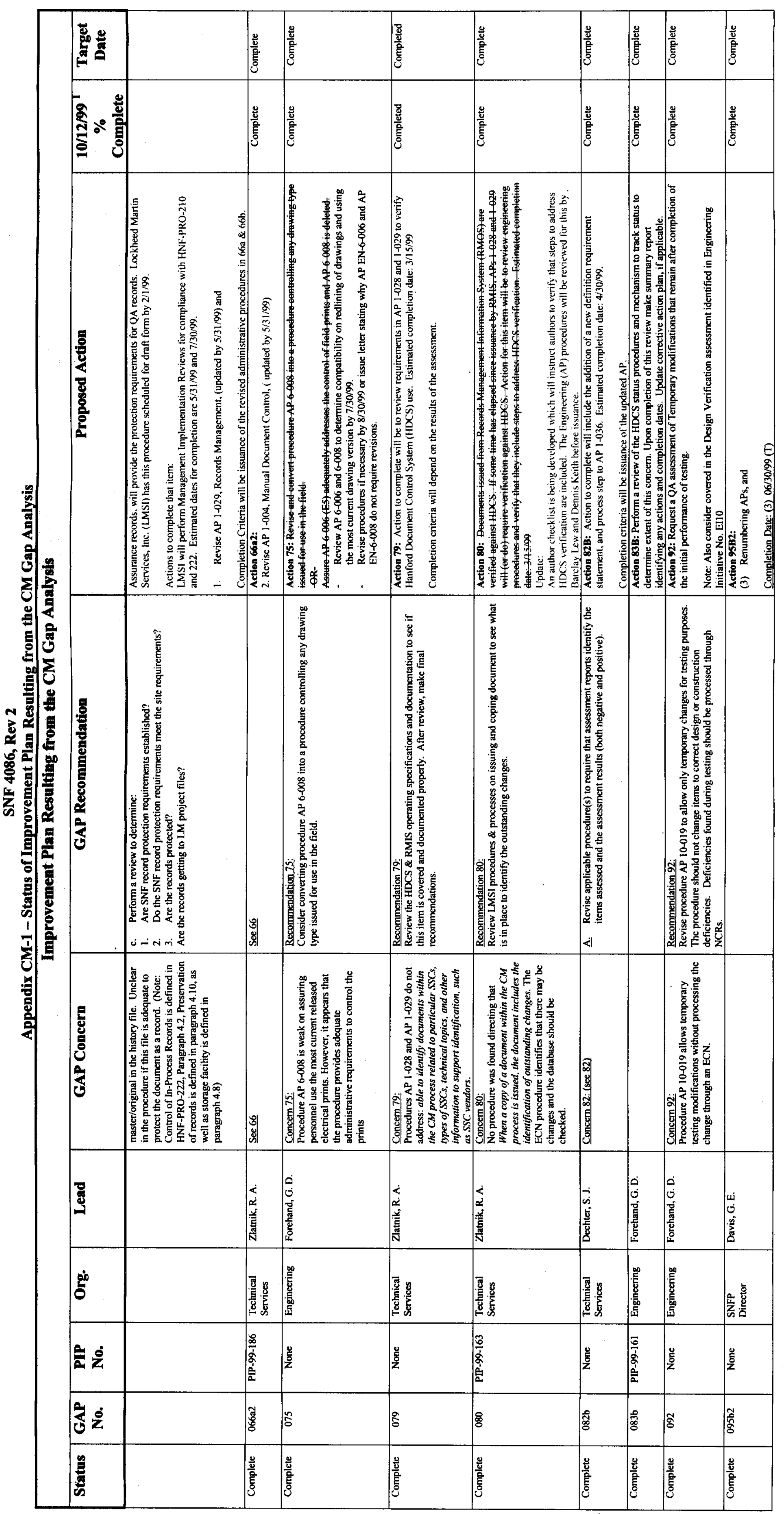




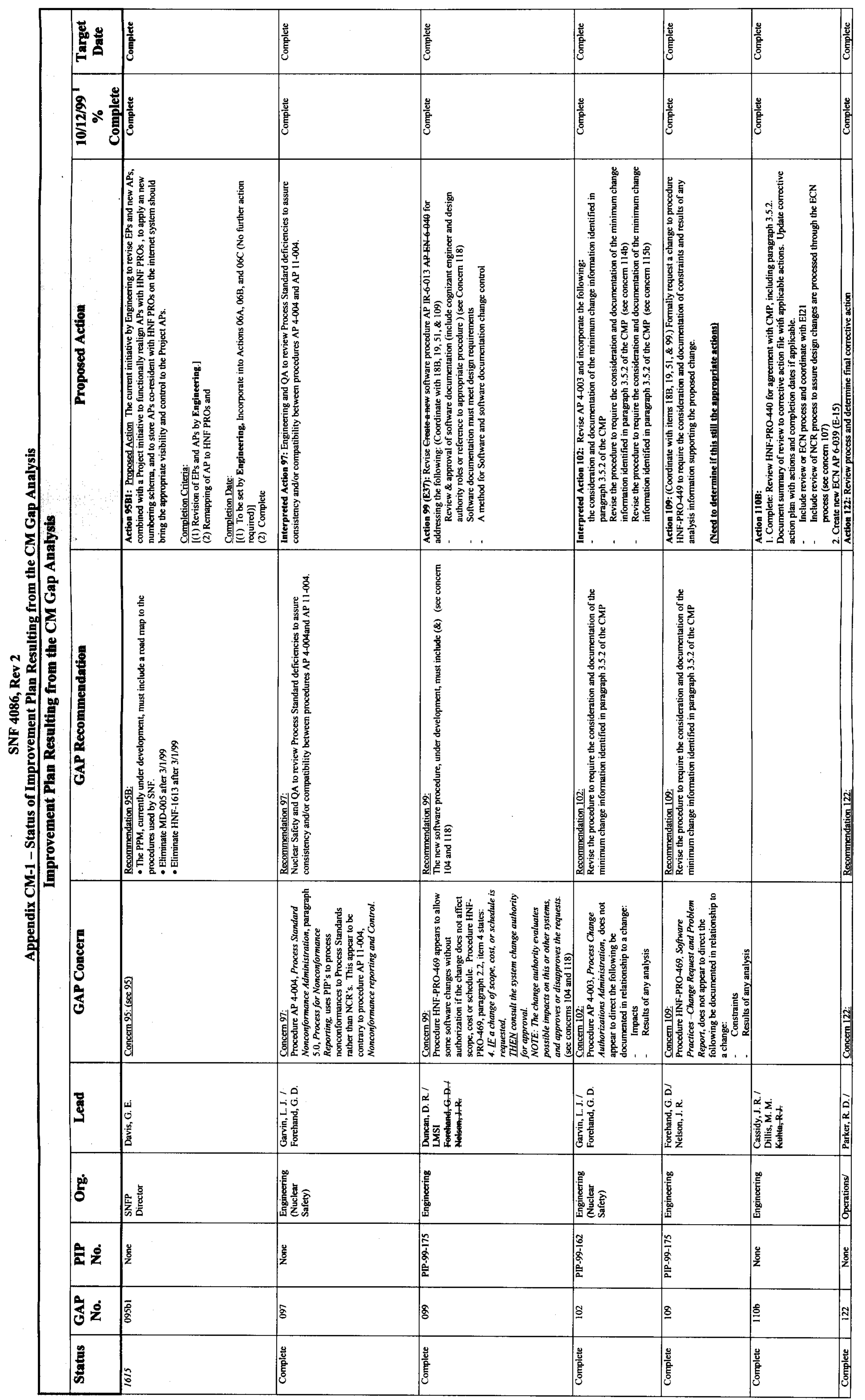




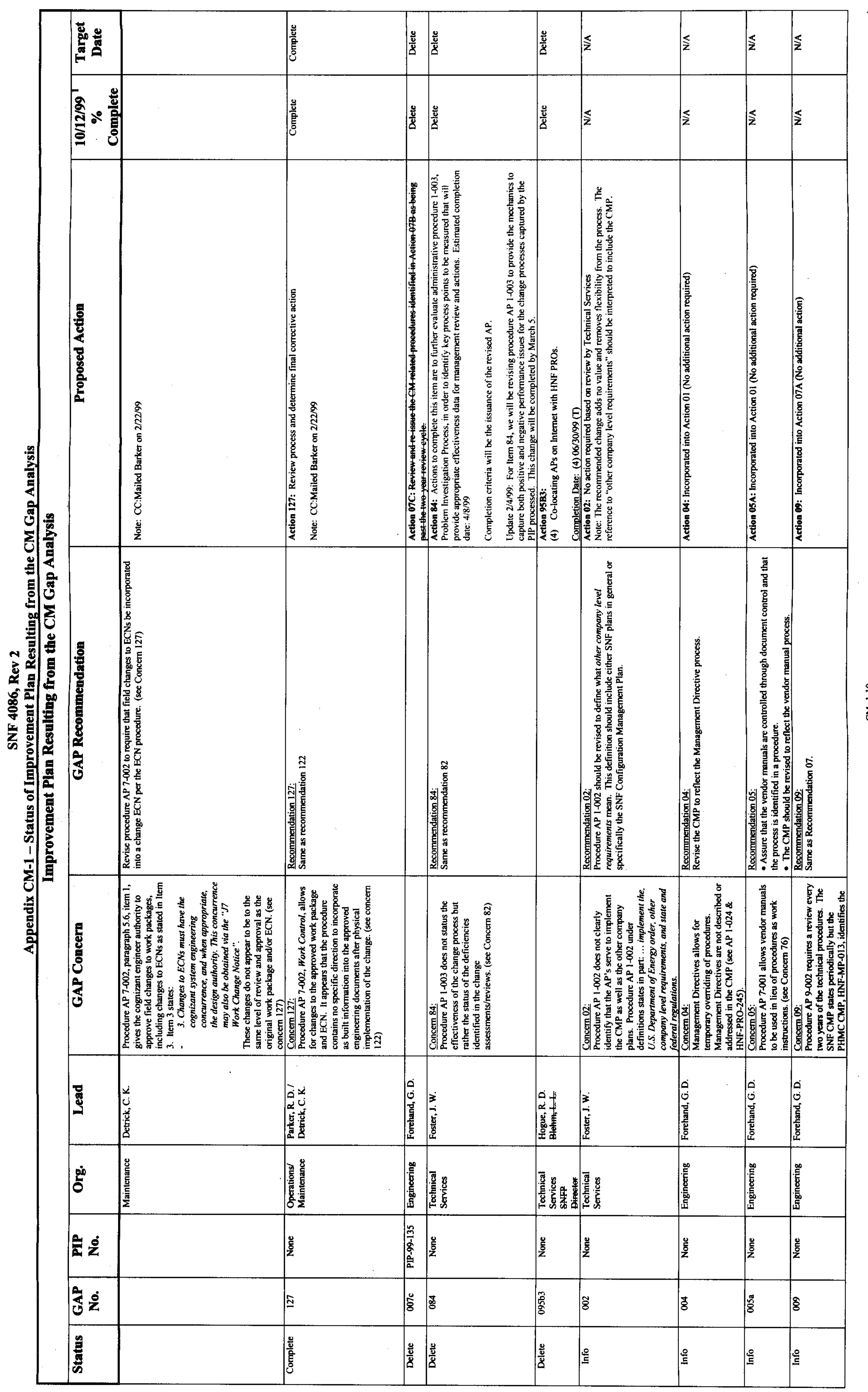




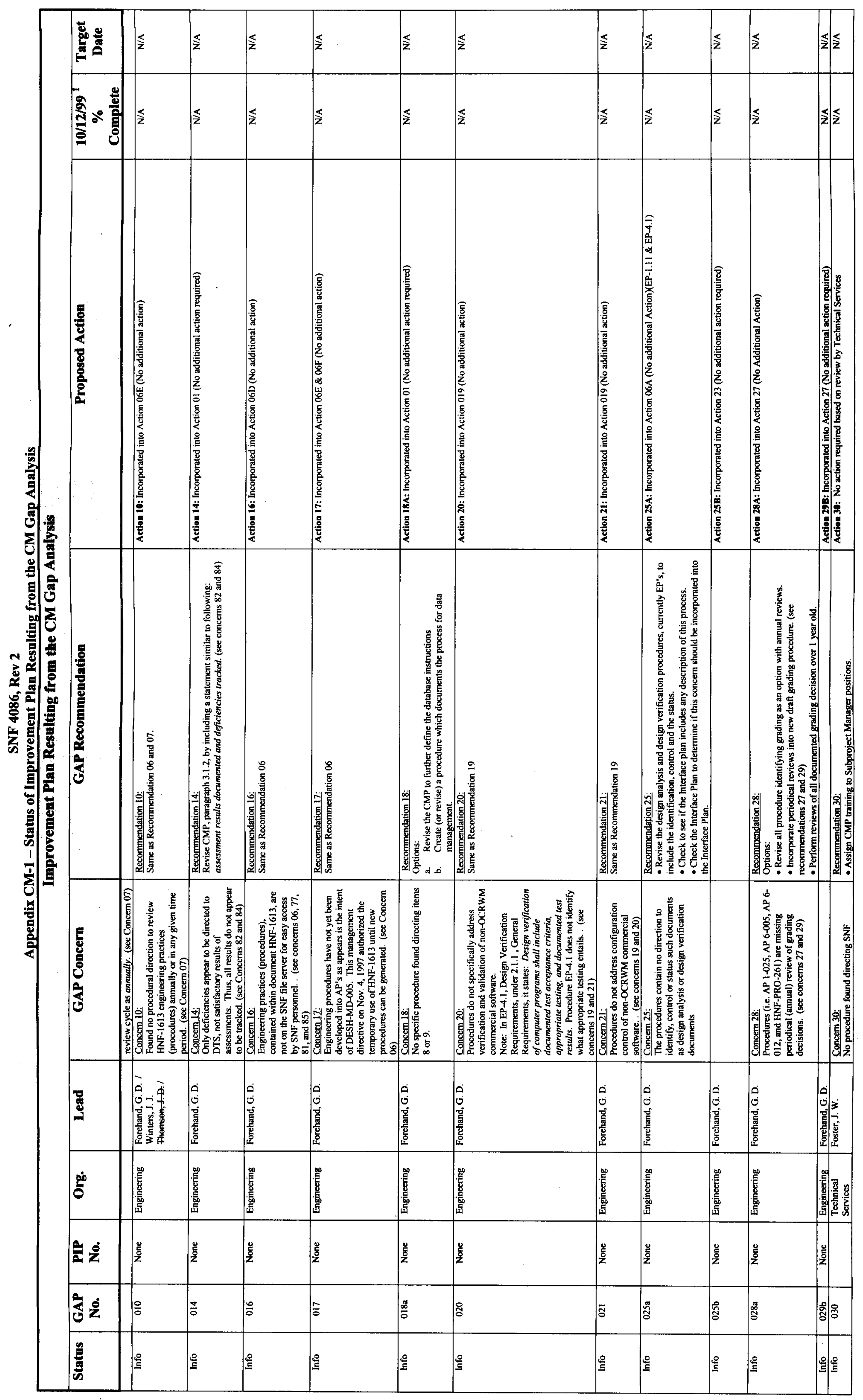




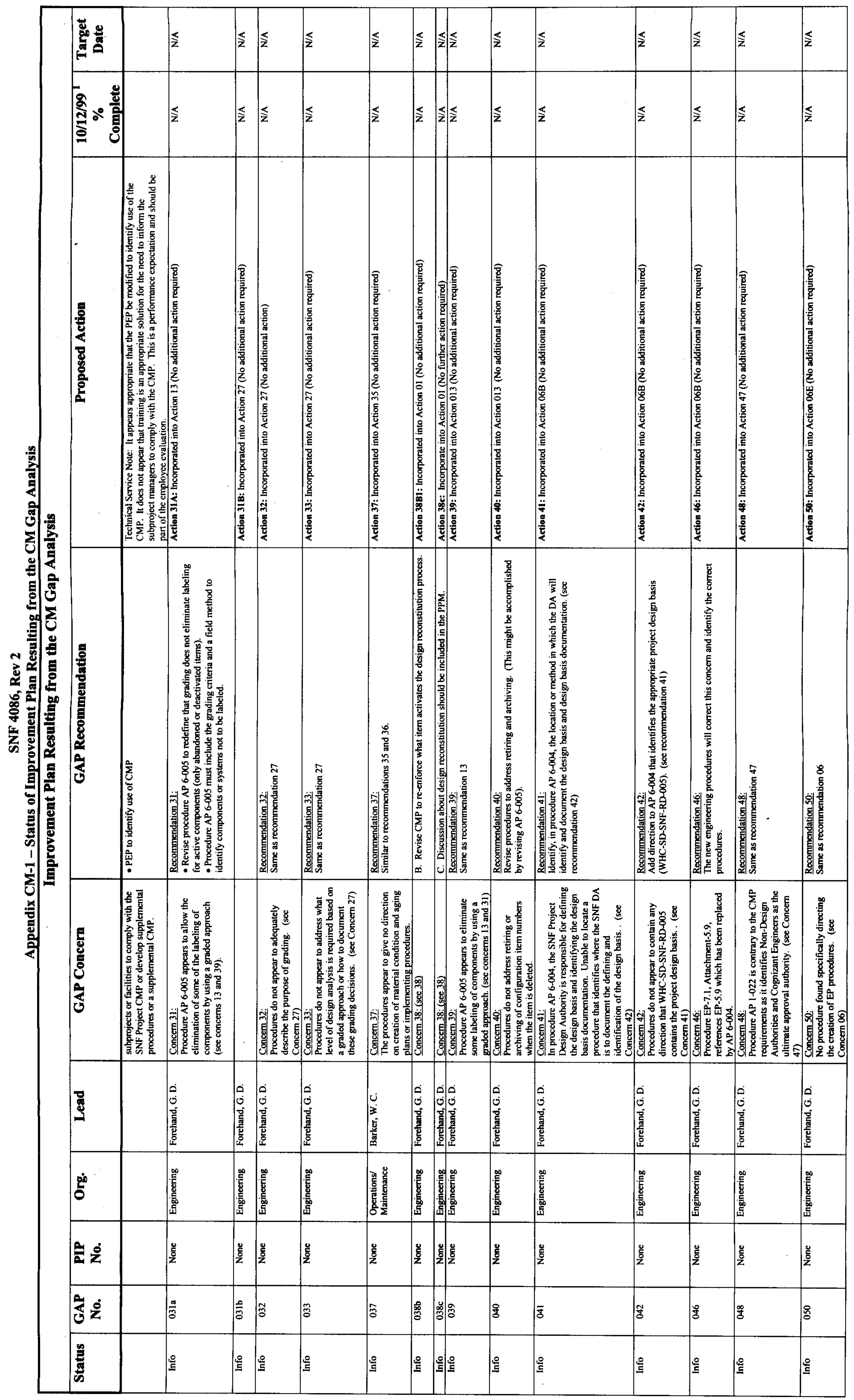




\begin{tabular}{|c|c|c|c|c|c|c|c|c|c|c|c|c|c|c|}
\hline & 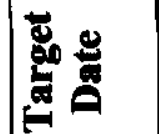 & $\frac{\mathrm{s}}{2}$ & 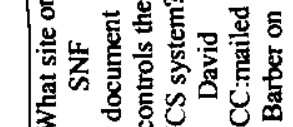 & s: & $\frac{\S}{\bar{z}}$ & $\frac{\hat{z}}{z}$ & & $\underline{\underline{z}}$ & $\frac{\hat{x}}{z}$ & $\frac{\hat{\Sigma}}{\bar{z}}$ & $\widehat{\aleph}$ & $\frac{\kappa}{z}$ & $\underline{\Sigma}$ & $\frac{\hat{x}}{2}$ \\
\hline & 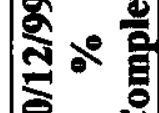 & $\frac{x}{z}$ & $\grave{z}$ & $\frac{\kappa}{z}$ & $\frac{\hbar}{z}$ & $\frac{5}{z}$ & & $\frac{\kappa}{z}$ & $\underline{z}$ & $\frac{\widehat{\Lambda}}{2}$ & $\frac{\Sigma}{z}$ & $\underline{\mathbf{z}}$ & $\underline{z}$ & $\frac{\kappa}{z}$ \\
\hline : & 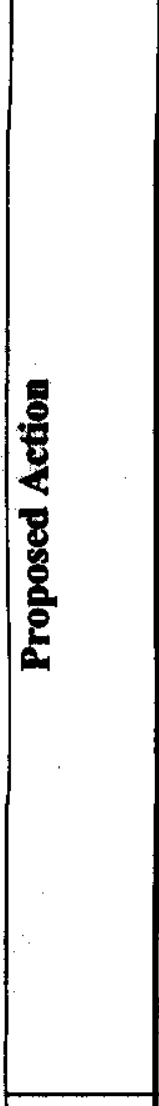 & 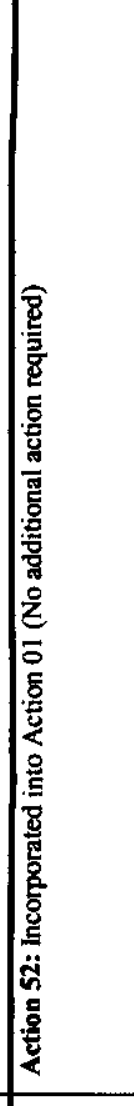 & 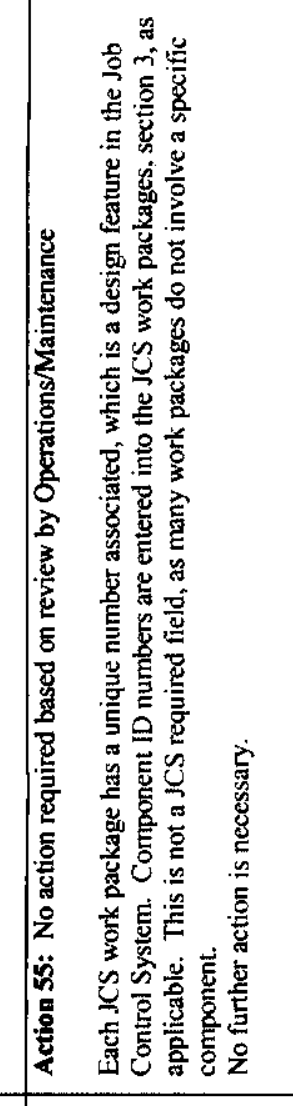 & 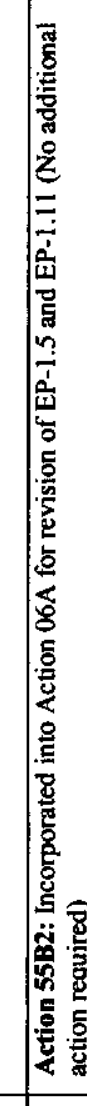 & 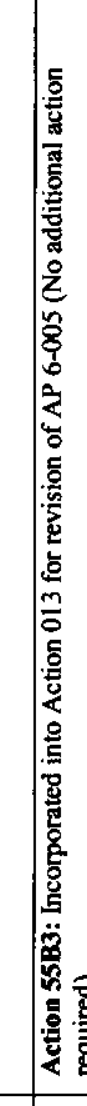 & 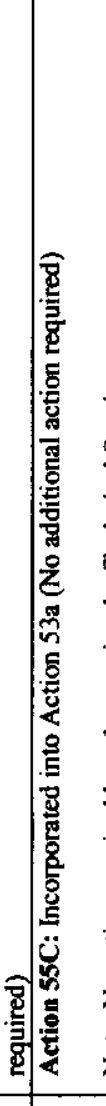 & 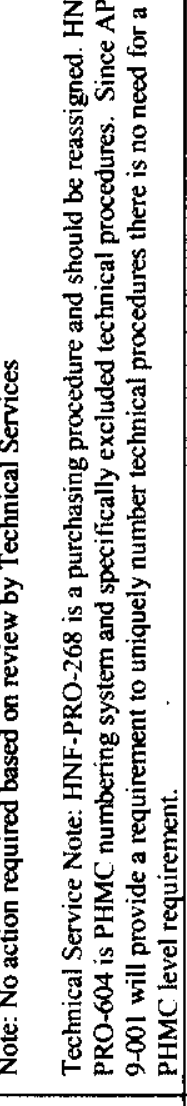 & 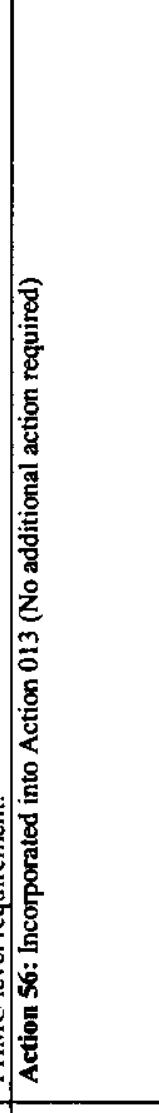 & 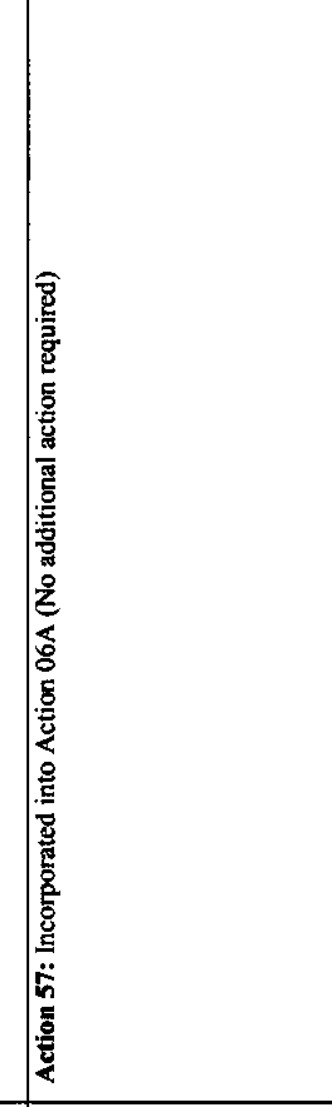 & 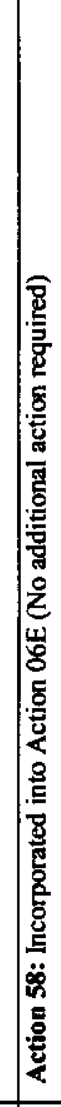 & 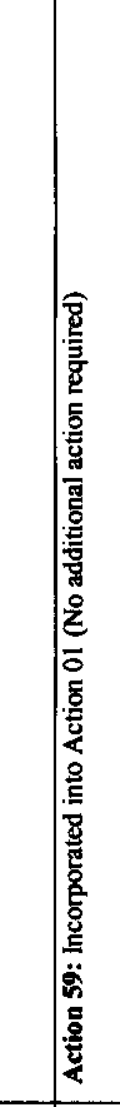 & 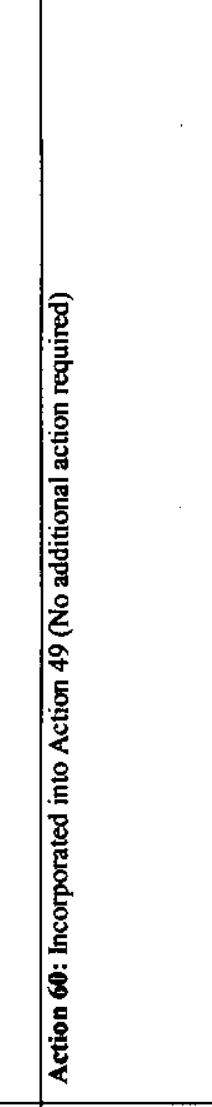 & 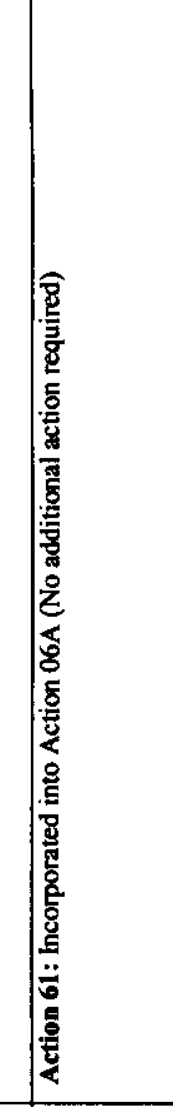 & 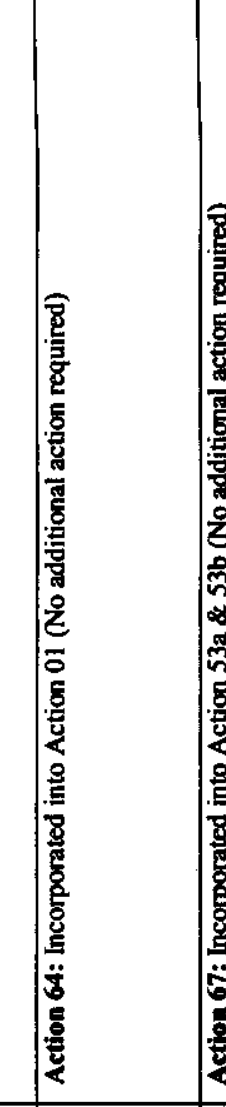 \\
\hline 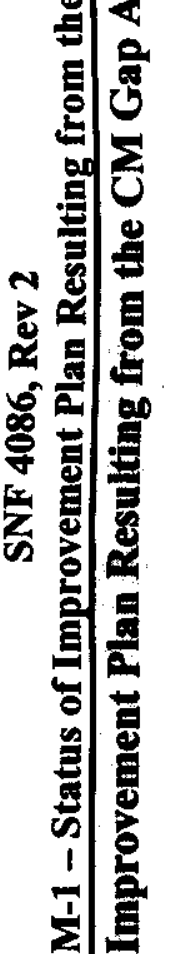 & 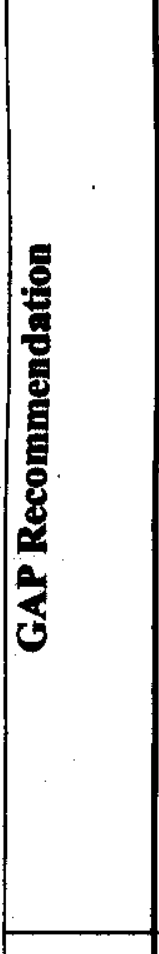 & 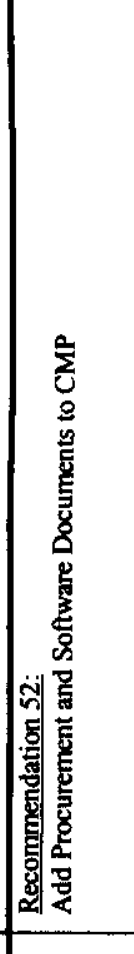 & 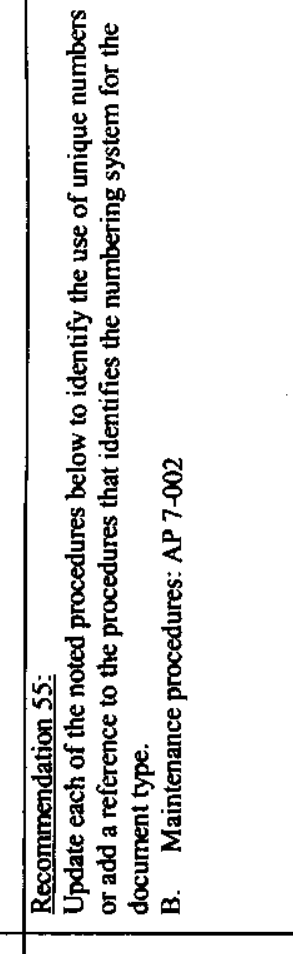 & & & 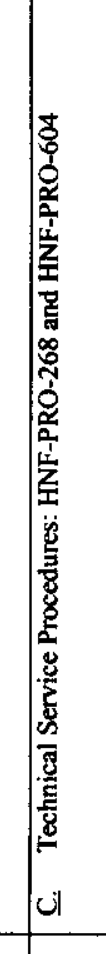 & & 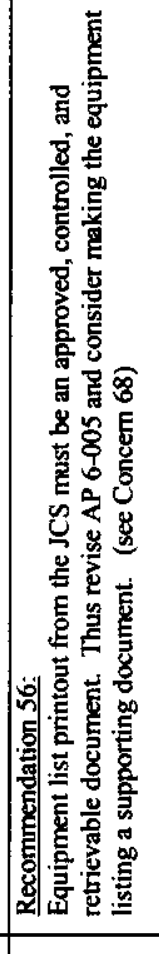 & 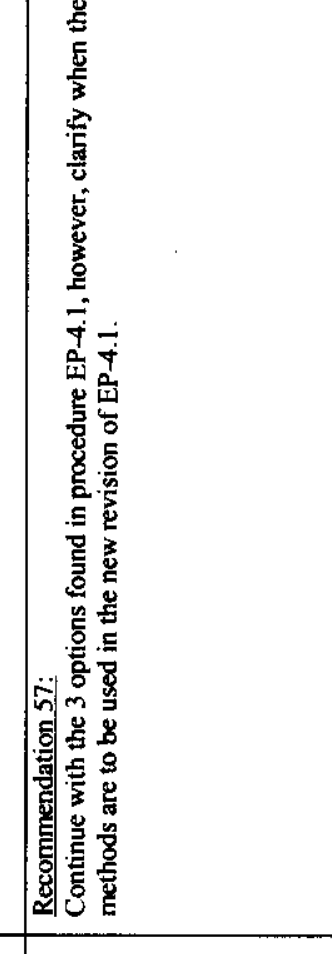 & : & 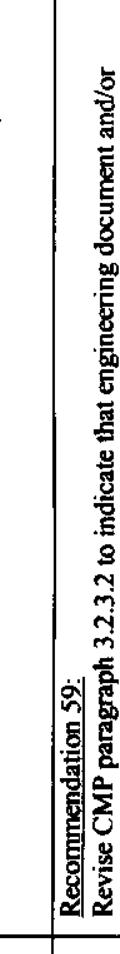 & & 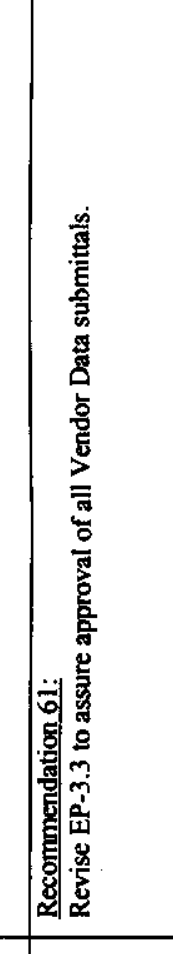 & 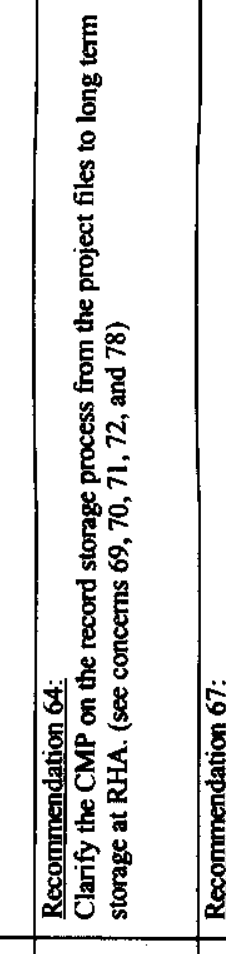 \\
\hline 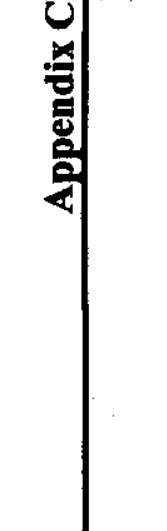 & 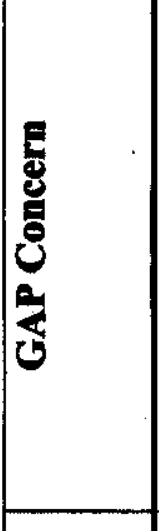 & 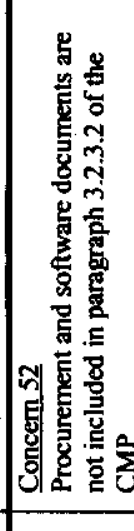 & 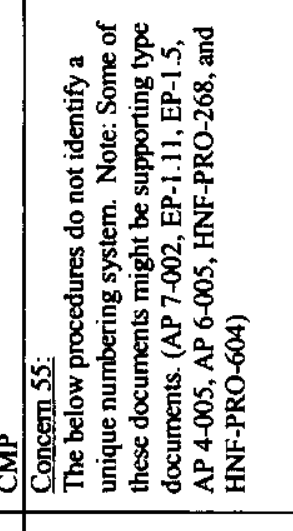 & & & & & 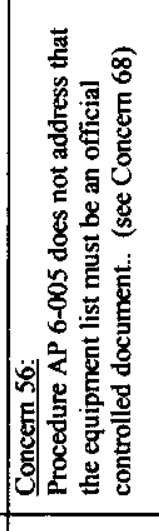 & 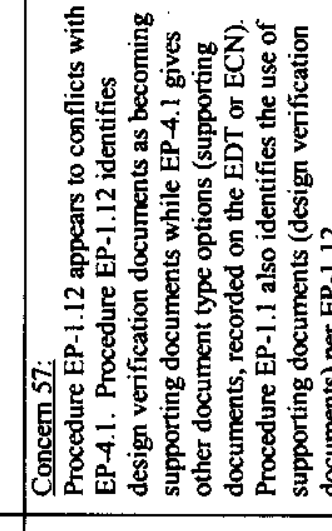 & & 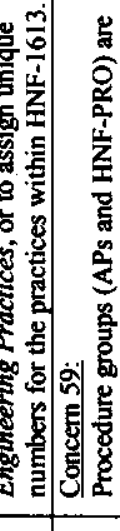 & 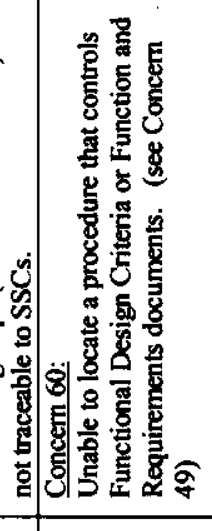 & 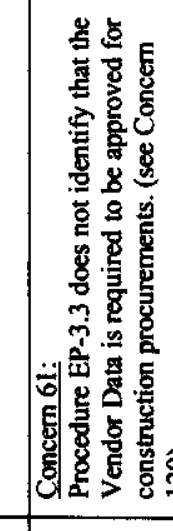 & 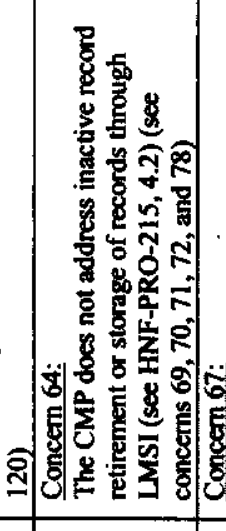 \\
\hline & 可 & 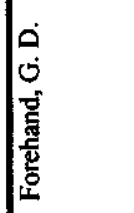 & 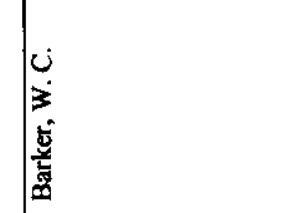 & 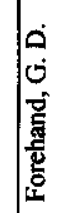 & 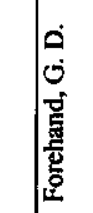 & 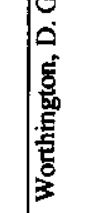 & & 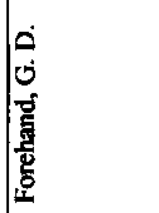 & 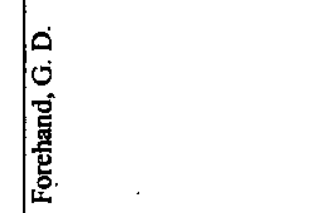 & 0 & 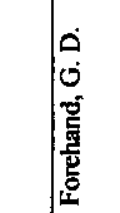 & 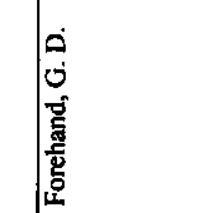 & 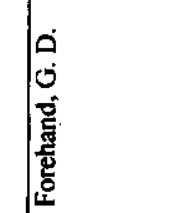 & 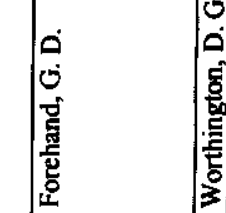 \\
\hline & to & $\mid$ & 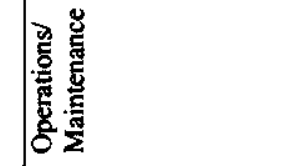 & 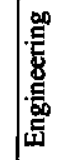 & & & & 澏 & 鄫 & & $\mid$ & $\mid$ & $\mid$ & $\mid$ \\
\hline & $\hat{\mathrm{a}}$ & 总 & 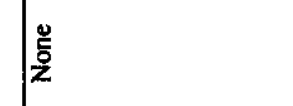 & 善 & 完 & 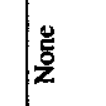 & & $\frac{\underline{z}}{2}$ & $\frac{0}{\frac{0}{2}}$ & 淁 & $\frac{\frac{0}{5}}{2}$ & $\frac{0}{\frac{5}{2}}$ & 竞 & $\frac{\frac{0}{5}}{2}$ \\
\hline & 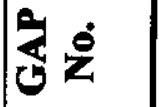 & $\tilde{8}$ & 咯 & 商 & 菅 & 污 & & $\because$ & $\tilde{s}$ & & oे & 8 & $\overline{8}$ & 吾 \\
\hline & 岂 & 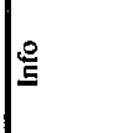 & $\stackrel{\circ}{\stackrel{\varrho}{E}}$ & 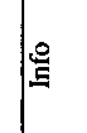 & & 总 & & $\stackrel{\varrho}{\Xi}$ & $\stackrel{0}{\underline{g}}$ & & 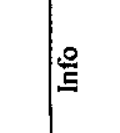 & 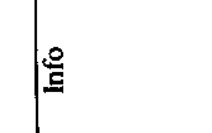 & $\stackrel{\circ}{\Xi}$ & 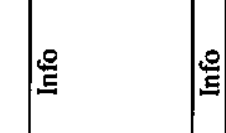 \\
\hline
\end{tabular}




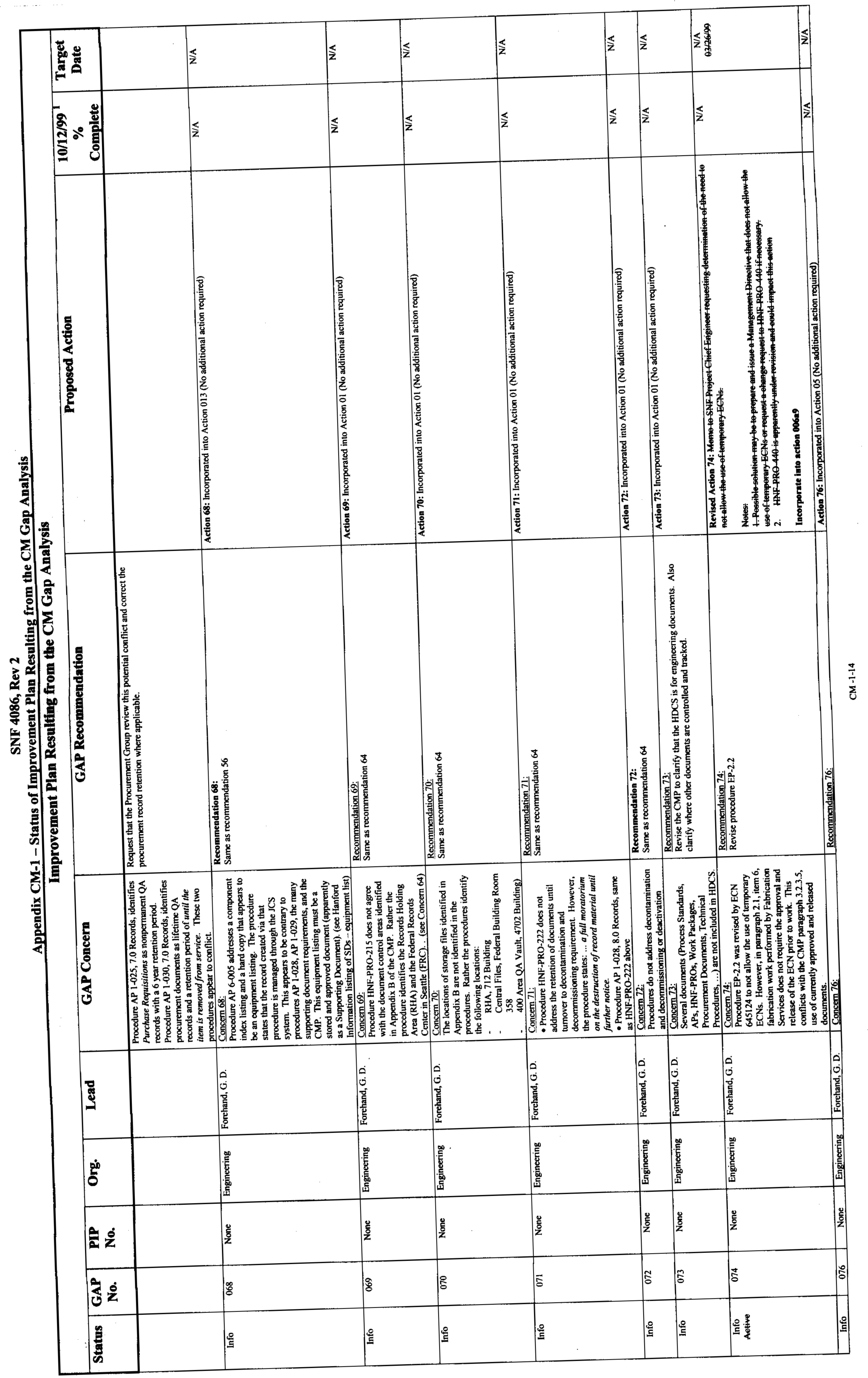




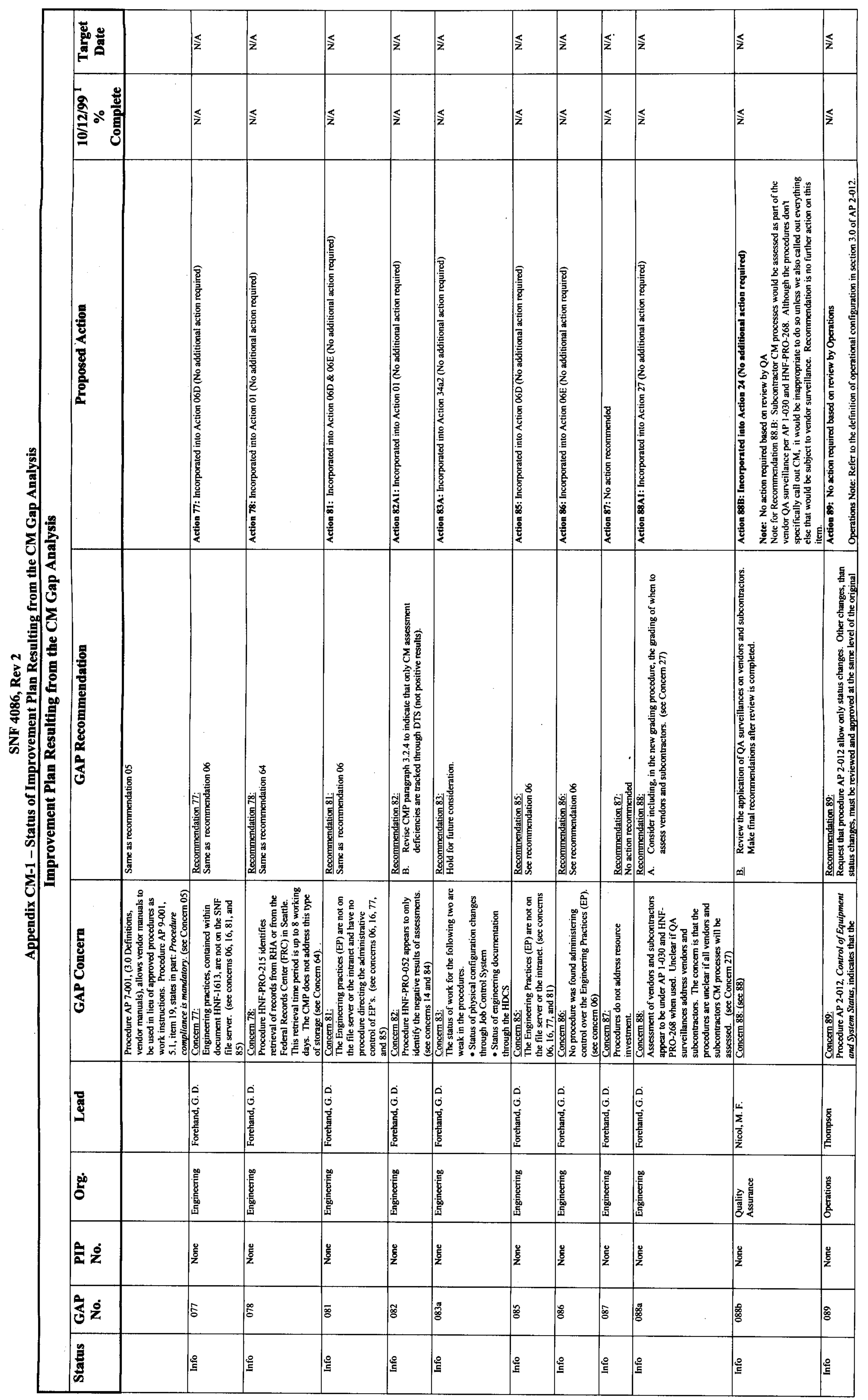




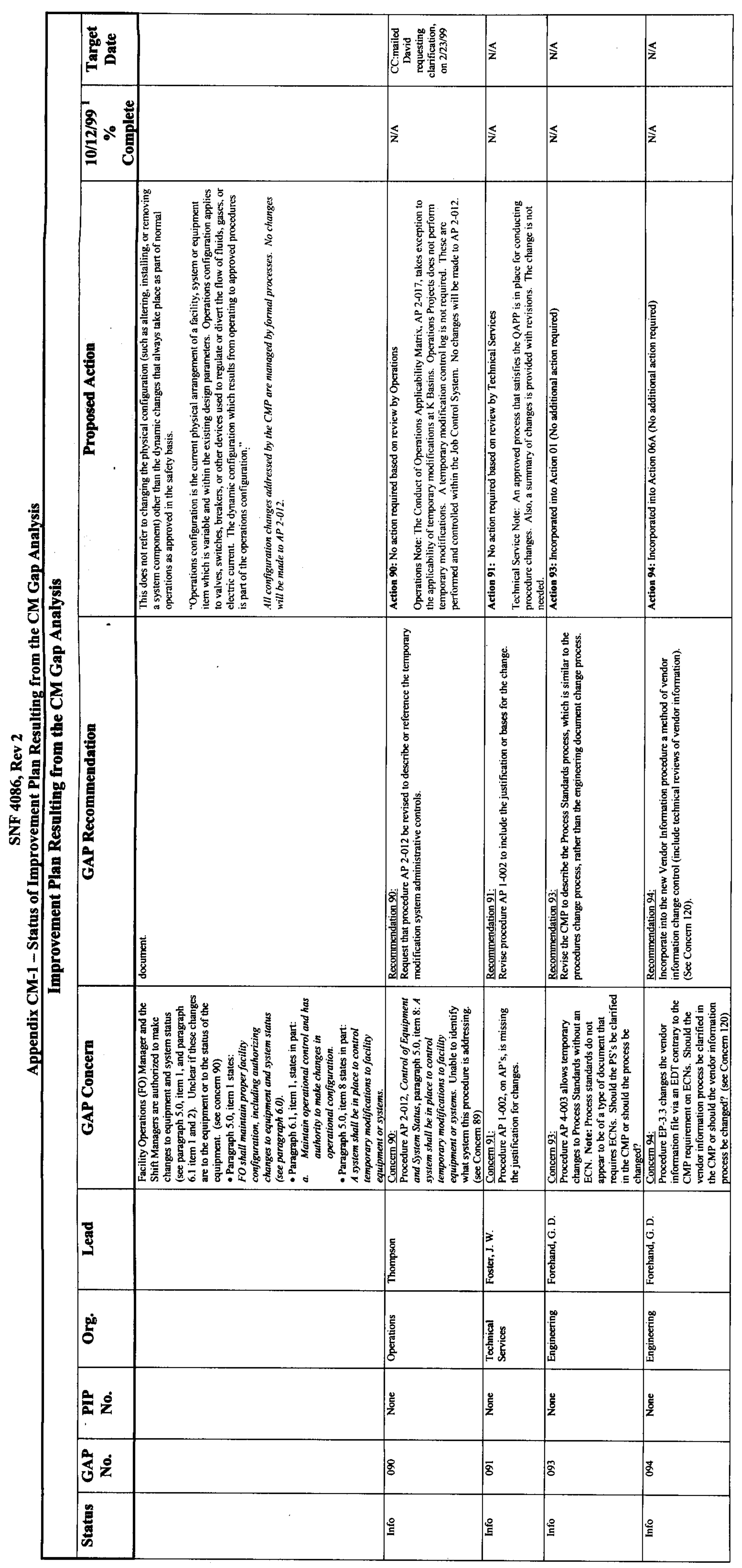




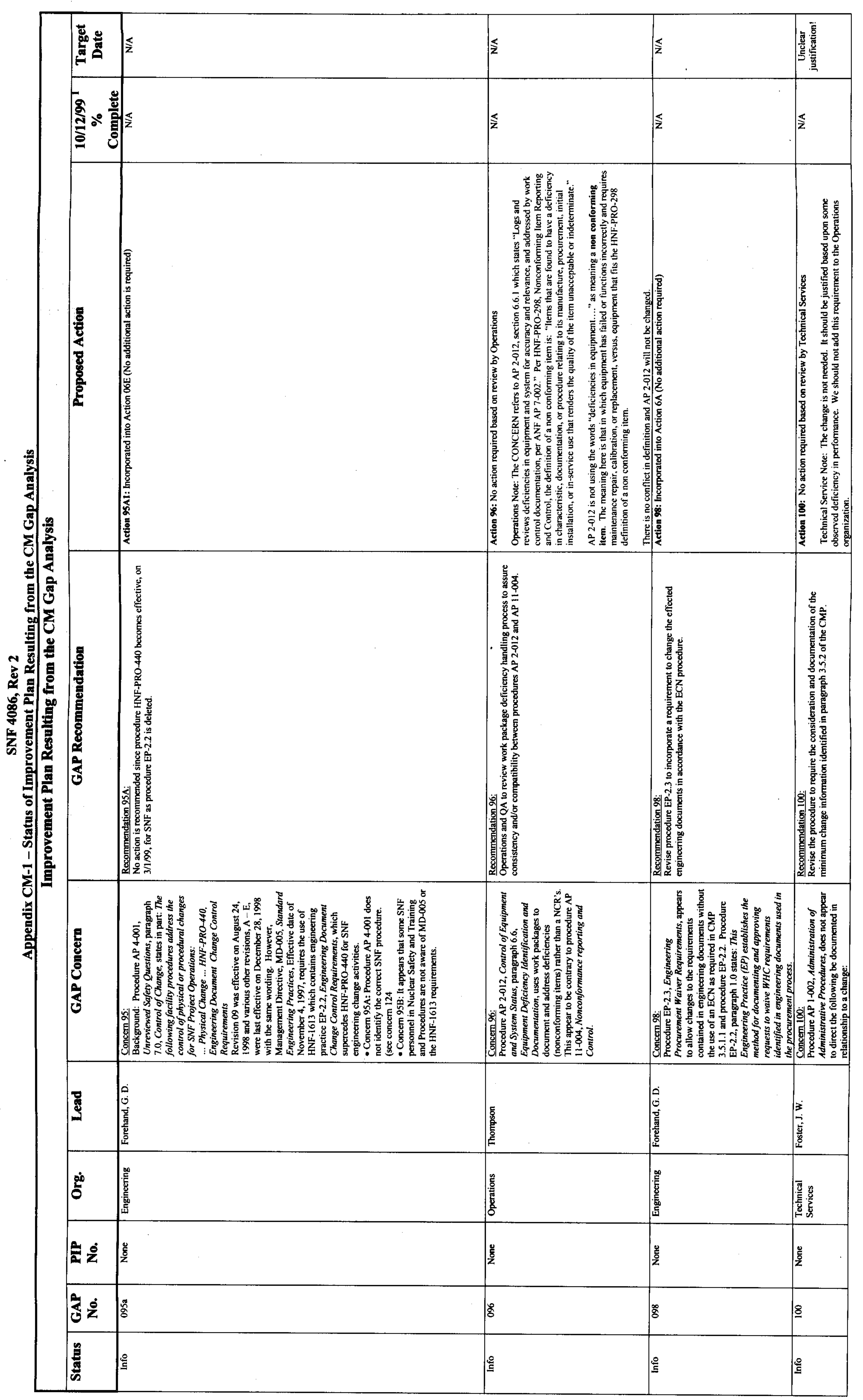




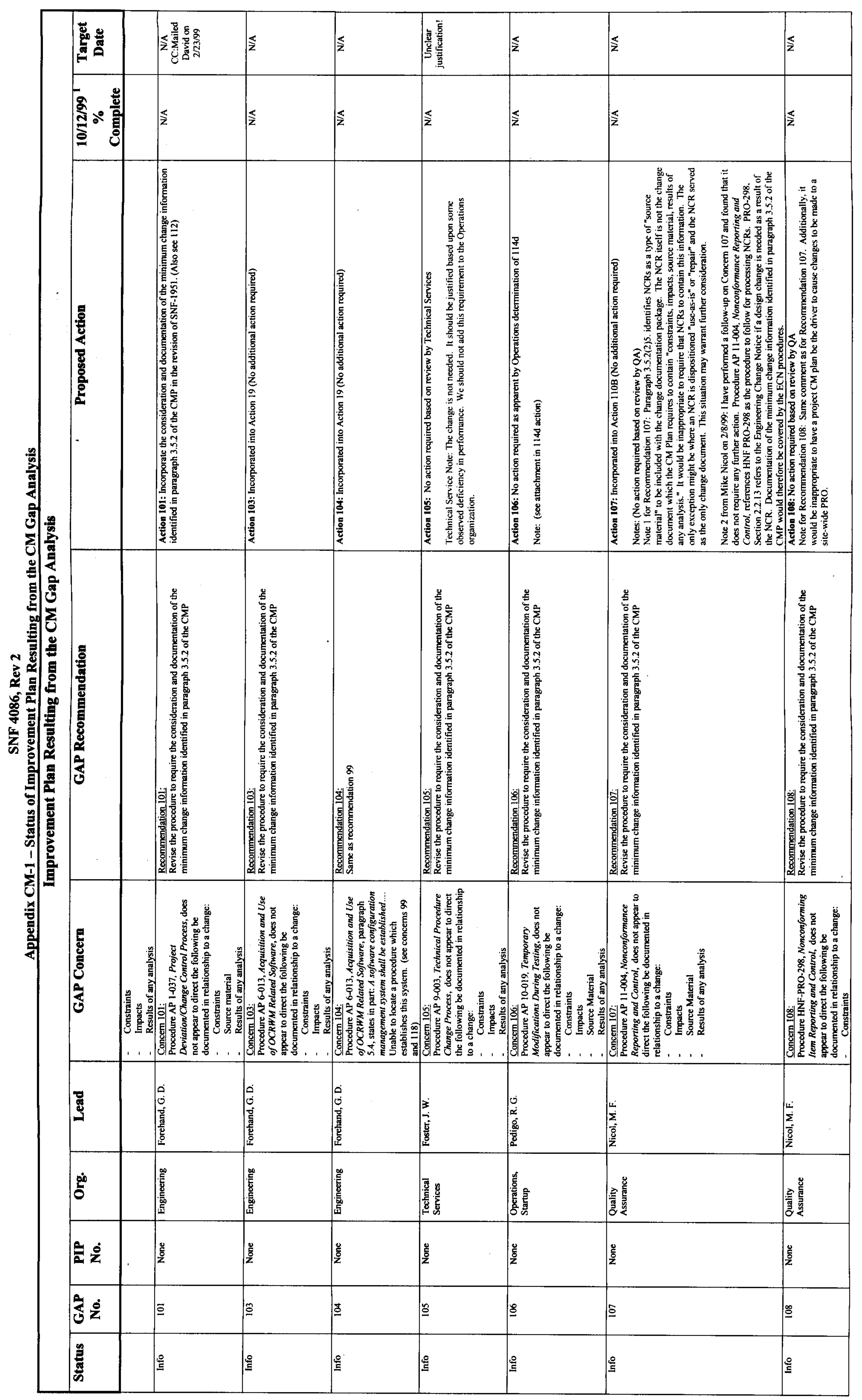




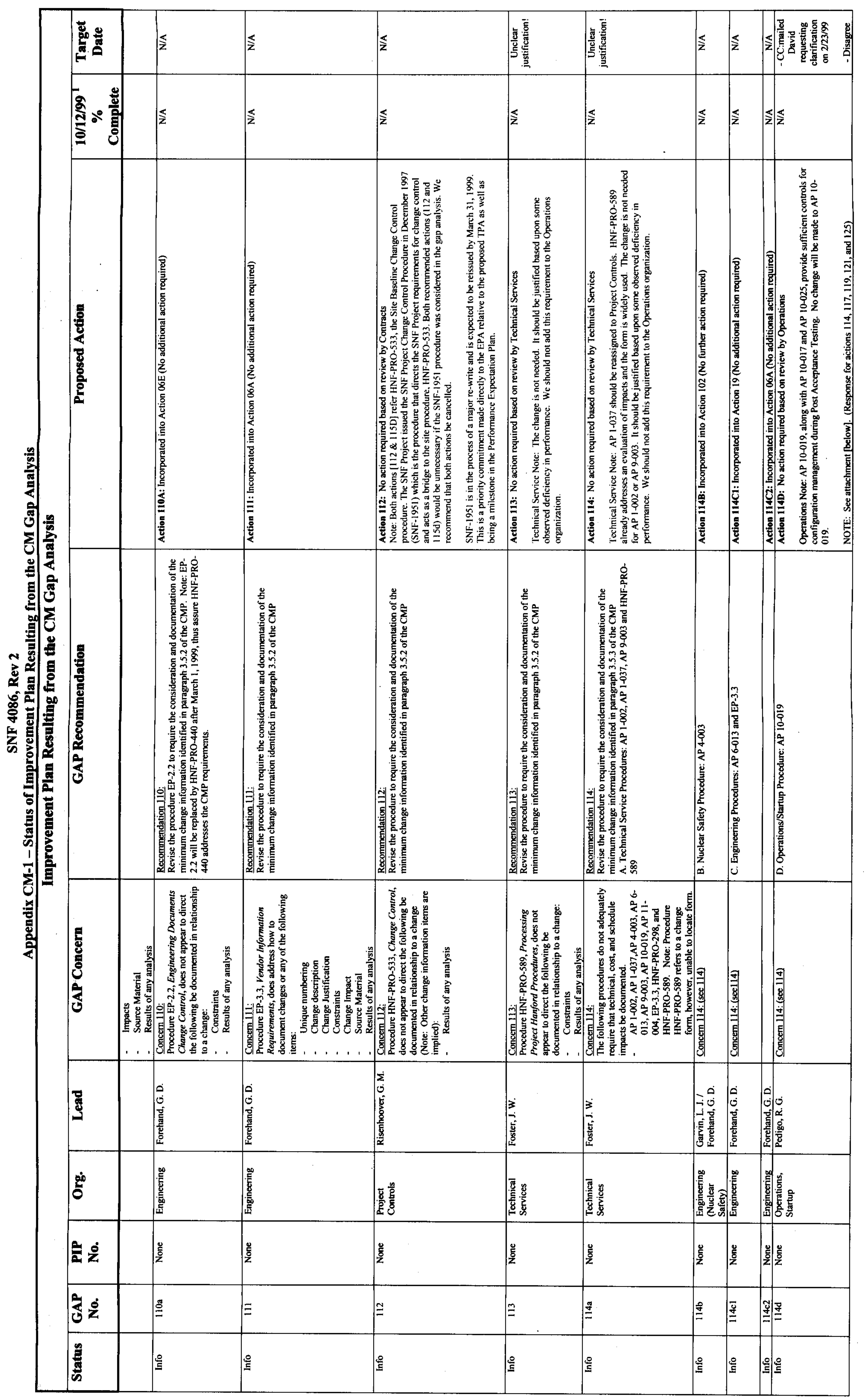




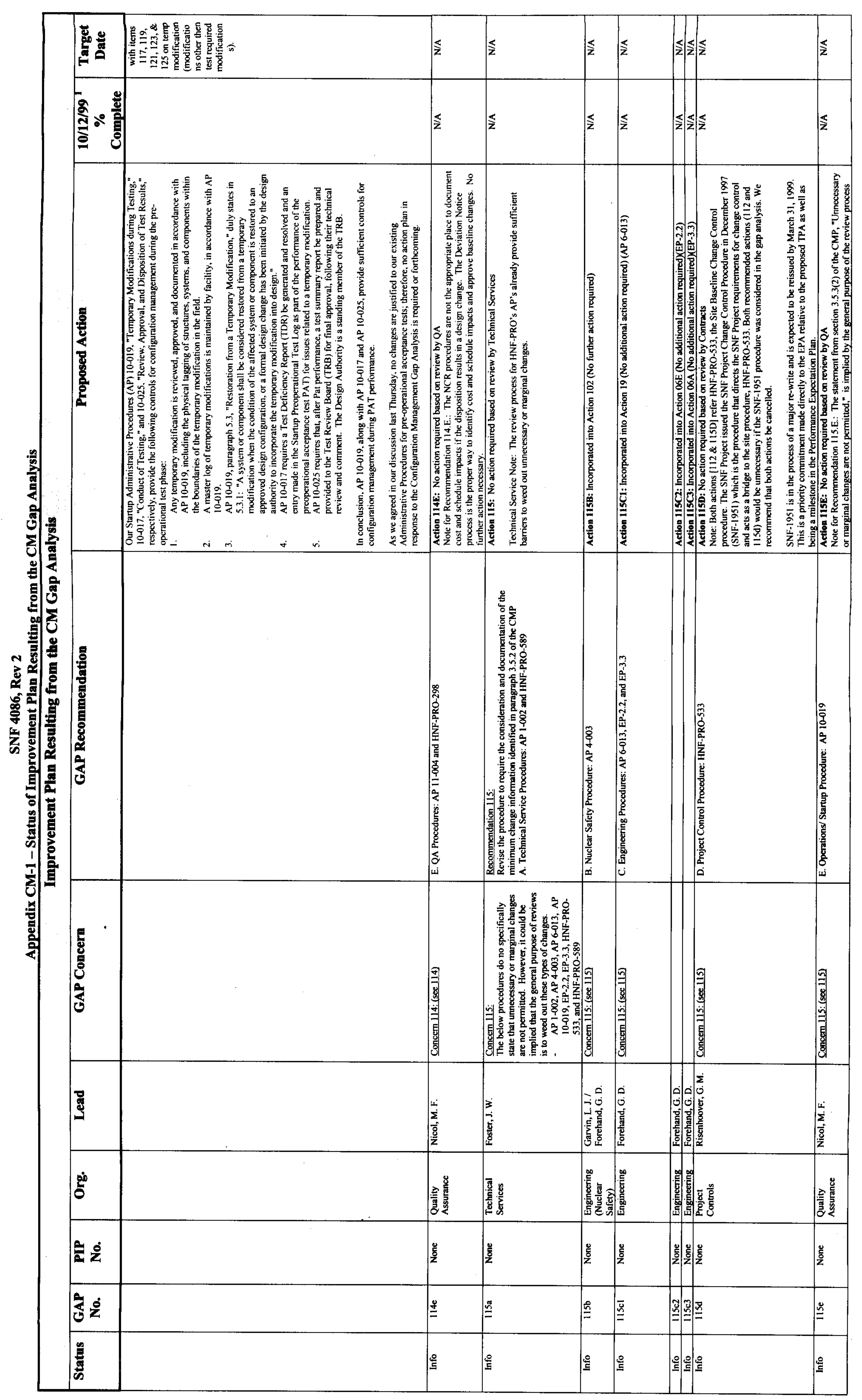




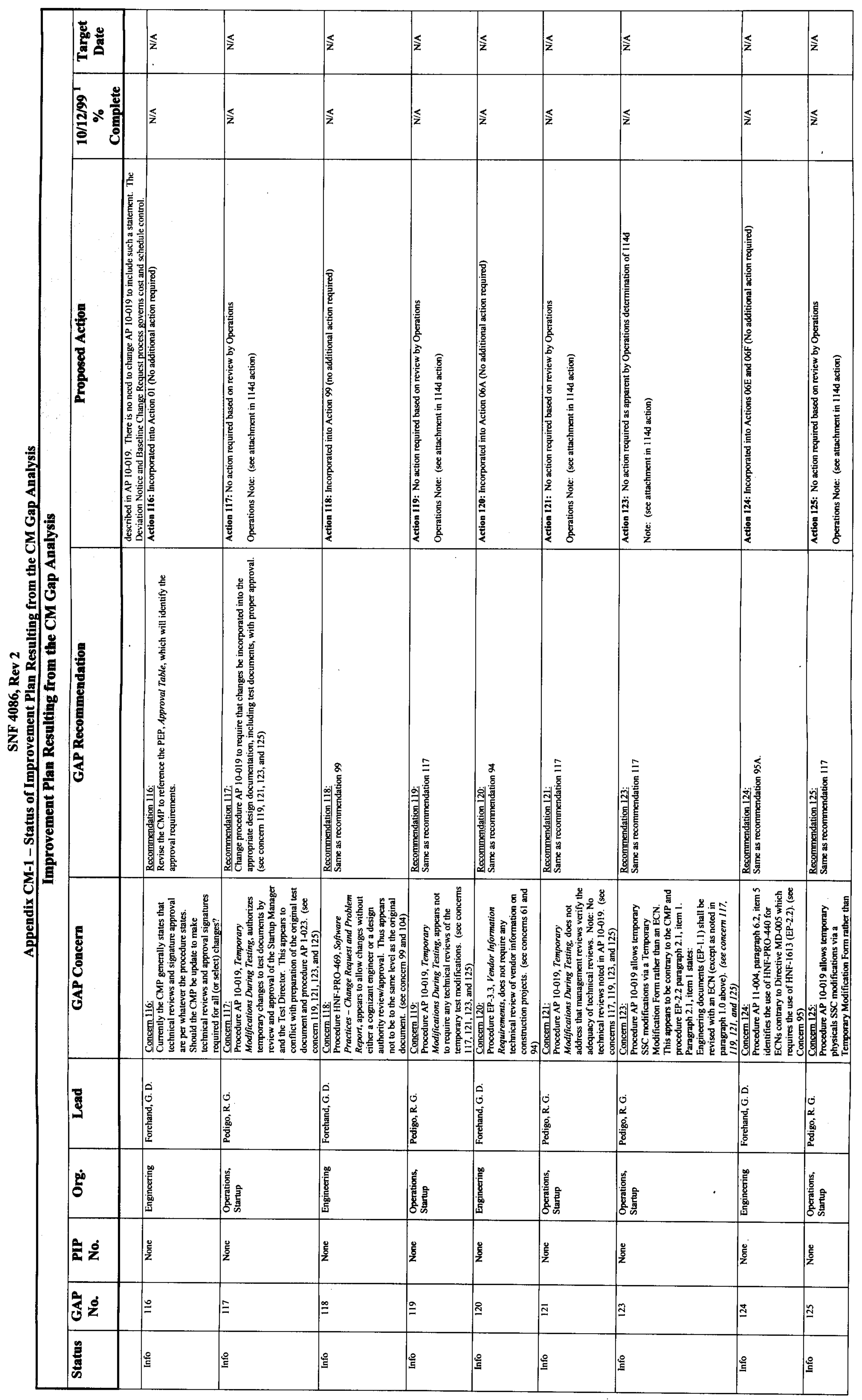




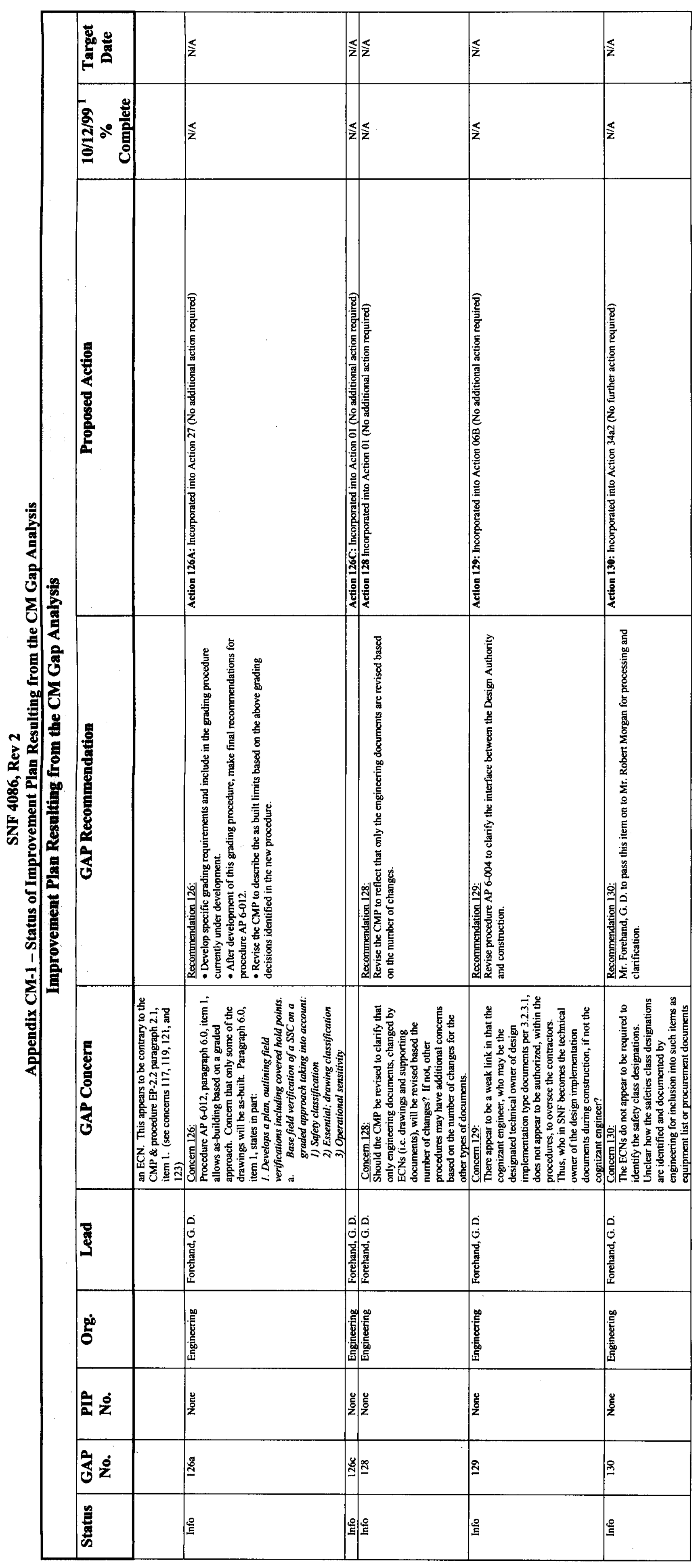




\section{SNF 4086, Rev 2 \\ Appendix CM-2 \\ Spent Nuclear Fuel Project \\ Closure/Status Report on Response FDH-9953258A R2 \\ to RL Assessment 99-AMW-015}

The following provides a status or closure of the 65 discrete actions identified in

Reference 1. These actions were undertaken in response to the two concerns and thirteen findings related to Spent Nuclear Fuel (SNF) Project Configuration Management (CM) implementation and corrective action management (CAM) activities identified in Reference 2, which provided the results of an integrated U.S. Department of Energy, Richland Operations Office (RL) assessment of the SNF Project's implementation of Reference 3.

The associated text (from Reference 2) for each concern or finding is in italics, followed by the corrective action summary in normal font. Supplemental documentation regarding the circumstances of each finding and concern and root cause information is provided in Reference 1. A complete summary of the status of all 65 corrective actions identified in Reference 1 is provided in Table 1. Supporting closure documentation is available in the Project files.

CONCERN: AMW-A-9901-C01

IN WORK The Appraisal Team is concerned with the reoccurrence of configuration management implementation deficiencies based on several recent examples of work being performed to unapproved design changes.

Actions have been completed to provide more effective tools to the Systems Engineering Manager when CM deficiencies are identified within the Spent Nuclear Fuel (SNF) Project corrective action process. This will provide a means for proactive management of these issues. The CAM organization has provided prototype reports on CM-related data from the Deficiency Tracking System (DTS) for the Systems Engineering Manager to evaluate. Secondly, a number of assessments were scheduled to monitor the $\mathrm{CM}$ /engineering process and have been included in self-assessment planning for fiscal year (FY) 2000. Some of the assessments and surveillances are currently in progress and will be completed by January 2000. An assessment of sub-project labeling progress was completed in November. In addition, procedural controls have been strengthened to impose the necessary CM controls on subcontractors. This action was a Fluor Daniel Hanford, Inc. (FDH) site-level EH-10 action and was confirmed complete via RL correspondence (Reference 4).

The third step was to brief project personnel on $\mathrm{CM}$ issues and the requirements for change control and design verification. This project-wide renewed commitment to CM and the extensive self-assessment process were designed to instill the importance of $\mathrm{CM}$ principles at all levels of the SNF Project.

In summary, a total of 13 of the 18 corrective actions for this concern have been completed. Those that remain include additional assessments planned for FY 2000 that 


\section{SNF 4086, Rev 2 \\ Appendix CM-2 \\ Spent Nuclear Fuel Project \\ Closure/Status Report on Response FDH-9953258A R2 \\ to RL Assessment 99-AMW-015}

will be integrated into the SNF Engineering self-assessment plan. A detailed schedule and budget for this work is being developed. This includes actions to track and verify all CM-related corrective actions. This will establish a final basis for closure of Concern C01.

CONCERN: AMW-A-9901-C02

CLOSED

The Corrective Action Management Process has not been effectively implemented on the SNF Project to address identified configuration management issues.

The CAM process has been strengthened by the site-wide implementation of HNF-PRO052 , Revision 2 . The latest revision of this procedure includes processes for evaluating new entries against existing entries to determine common causes, trends events at all levels, adds a process for evaluating corrective action effectiveness, and manages the timeliness of corrective actions. The CAM process will also be used to generate SNF Project CM specific reports that management can use to ensure no systemic problems are evident.

Two actions for this concern were FDH site-level EH-10 actions. The action to implement DTS and CAM systems was confirmed complete via RL correspondence (Reference 4). The second action was to perform an assessment to ensure these corrective actions are effective in resolving the issues. This assessment was completed and the Facility Evaluation Board's final report has been sent to RL as part of Reference 5.

The project-wide communications regarding the importance, benefits, and expectations of the CAM system, the CAM Process improvements, and the assessment all serve as a basis to close Concern $\mathrm{CO} 2$.

All ten corrective actions for this concern have been completed.

FINDING: AMW-A-9901-F01

CLOSED

The implementation of the Corrective Action Management Process for deficiencies identified during receipt of electrical support equipment for the MHM did not meet requirements. Specifically, it did not identify the recurring nature of the fundamental issue, the root cause/causes, and corrective actions addressing root causes.

This finding was addressed with the revision of the Multi-Canister Overpack (MCO) Handling Machine (MHM) Performance Specification and actions taken by the Chief Engineer to reemphasize to all engineering and projects personnel that formal documentation is required for all contractual agreements. In addition, the CM and CAM 


\section{SNF 4086, Rev 2}

FDH-9953528A R3 Attachment

\section{Appendix CM-2}

Spent Nuclear Fuel Project

Closure/Status Report on Response FDH-9953258A R2

to RL Assessment 99-AMW-015

processes have both been improved through the actions discussed for Concerns $\mathrm{C} 01$ and $\mathrm{C} 02$.

Both corrective actions for this finding have been completed.

FINDING: AMW-A-9901-F02

CLOSED

The implementation of the Corrective Action Management Process for deficiencies identified by the Government Acceptance Inspector (AI) during system inspections at $C V D F$ did not meet requirements. Specifically, the investigative report did not identify the recurring nature of the fundamental issue, the root cause/causes, corrective actions addressing the specific deficiencies, and was not issued in a timely manner.

The root cause analysis identified the need for the Cold Vacuum Drying (CVD) SubProject Manager to prioritize or allocate resources so that contractual notification was effectively updated to support construction activities. Contributing factors were a deficiency in administrative controls and procedures did not specifically indicate that critique results must be entered into the CAM process.

In response to this finding, actions were implemented to ensure a more timely change process for Design Change Notices (DCNs), and to allocate the resources necessary to ensure regular review of actions (e.g., DCNs) required for the support of ongoing/upcoming construction activities. In addition, work control processes have been modified to ensure that critique results are included in the CAM process, and the original critique was revisited to ensure that all issues were resolved. The former action was an FDH site-level EH-10 action and was confirmed complete via RL correspondence (Reference 4).

All three corrective actions for this finding have been completed.

FINDING: AMW-A-9901-F03 CLOSED Configuration management deficiencies were not corrected in a timely manner and actions were not taken to determine the root cause and extent of the identified problem as required.

The CVD Sub-Project Manager authorized funding to ensure adequate root cause evaluations were carried out and to implement a tracking process for non-conformance reports, deficiency reports, and design changes. A primary function of this tracking system will be to ensure timely closure of corrective actions and to discourage commitment date extensions. 
SNF 4086, Rev 2

Appendix CM-2

Spent Nuclear Fuel Project

Closure/Status Report on Response FDH-9953258A R2

to RL Assessment 99-AMW-015

Both corrective actions for this finding have been completed. 
SNF 4086, Rev 2

FDH-9953528A R3 Attachment

Appendix CM-2

Spent Nuclear Fuel Project

Closure/Status Report on Response FDH-9953258A R2

to RL Assessment 99-AMW-015

FINDING: AMW-A-9901-F04

CLOSED

Design interfaces for the MCO have not been properly identified and controlled as

required by 10 C.F.R. 830.120 .

- MCO Interface Table has not been updated

- There is a lack of Interface Control Screening on DCNs/ECNs

- The SNF Project file does not have records of the monthly Interface Control Database (ICD)

The MCO Interface Table is being maintained through an action item management process assigned by charter to the Interface Control Working Group. In addition, several actions have been implemented project-wide to enhance interface control practices for all subprojects. First, a new interface control procedure has been issued. This has been augmented by enhanced Design Authority training activities that include emphasis on effective interface control practices and the enforcement of procedural requirements. All Design Authorities have attended briefings on the new revision of the interface control procedure.

In addition, a surveillance of Engineering Change Notices (ECNs) with the potential to impact interfaces was completed in October 1999. The information gathered from this surveillance will aid in the planning of future interface process improvements.

Both corrective actions for this finding have been completed.

FINDING: AMW-A-9901-F05

CLOSED

Construction work is being performed on the CVDF to design changes documented on Requests for Information (RFI) instead of on approved DCNs, as required.

The root cause of this finding identified the need for more effective enforcement of the desk instruction and policy for proper use of RFIs. To rectify this situation, the CVD Sub-Project Manager issued correspondence directing project personnel to stop using RFIs for design changes, and to reiterate the requirement to use DCNs to implement all design changes. In addition, the project manager directed the Architect/Engineer (A/E) to evaluate all past RFIs to identify any used for design changes. As a result of this evaluation, the $\mathrm{A} / \mathrm{E}$ executed recovery actions to develop a $\mathrm{DCN}$ for all the noted design changes.

All three corrective actions for this finding have been completed. 
SNF 4086, Rev 2

FDH-9953528A R3 Attachment

Appendix CM-2

Spent Nuclear Fuel Project

Closure/Status Report on Response FDH-9953258A R2

to RL Assessment 99-AMW-015

FINDING: AMW-A-9901-F06

CLOSED

Configuration management deficiencies at $K$ Basins were not corrected in a timely manner, nor were actions taken to determine the extent of identified problems.

Resources were allocated to complete the labeling actions necessary to close PIP 97-389 and PIP 98-161. This included the crafts to prepare the labels and a craftsperson and operator for label installation. An engineer then verified the label installation. In addition, Administrative Procedure EN-6-005 was revised to provide improved guidance for labeling activities, including a graded approach to labeling.

While completing the commitments for this finding, this graded approach was implemented by the SNF Project Facility Engineering Manager (Mr. J. R. Cassidy) and RL (Mr. K. M. Schierman and Mr. J. M. Escamillo) to reach agreement on delaying scheduled installation of some labels. This was done for as-low-as-reasonablyachievable (ALARA) reasons since installation of some labels required scaffolding in potentially contaminated areas. In addition, these actions will be evaluated as part of the self-assessment process that has been implemented by SNF Engineering.

All three corrective actions for this finding have been completed.

FINDING: AMW-A-9901-F07

CLOSED

SNF Project drawing revisions referenced in work packages in use to support field activities are not consistent with Technical Data Center (TDC) records in use by project personnel as required.

SNF Contracts has reviewed the current SNF Project K Basin TDC wherein a direct communication link with the document control department of the specific Integrated Water Treatment System (IWTS) vendor (Chem-Nuclear Systems, Inc. [CNSI]) has been established. This newly revised process has resulted in the TDC receiving the design documentation directly from the vendor's Document Control and has the capability to control the design documents ensuring that only current revisions are in use.

Based on SNF Contracts review of other existing contracts, there are no other cases where a document control issue like that experienced with CNSI can occur. Therefore, this finding can be closed. It should be noted that this experience is being used as a lessons-learned topic and is being utilized to establish expectations with vendors on all new contracts.

The corrective action for this finding has been completed. 
SNF 4086, Rev 2

Appendix CM-2

Spent Nuclear Fuel Project

Closure/Status Report on Response FDH-9953258A R2

to RL Assessment 99-AMW-015 
FDH-9953528A R3 Attachment

\section{SNF 4086, Rev 2 \\ Appendix CM-2 \\ Spent Nuclear Fuel Project \\ Closure/Status Report on Response FDH-9953258A R2 \\ to RL Assessment 99-AMW-015}

FINDING: AMW-A-9901-F08

CLOSED

ECNs were not being incorporated (by revision) into drawings within required periodicity.

The Facility Engineering Manager undertook a number of actions to prevent similar events from taking place in the future. First, the Facility Engineering Manager ensured that staff were briefed on the requirements for incorporating ECNs in a timely fashion. All Facility Engineering personnel were required to attend a briefing on administrative procedure (AP) EN-6-006. Second, the Facility Engineering Manager communicated his expectations on the continuity of drawing-related activities throughout personnelturnover events via internal memo to all engineering staff. This memo also included his commitment that engineering resources will be prioritized to ensure effective maintenance of both essential and support drawings. Finally, Revision 0 of a controlled support drawing list was issued in June 1999. The tangible results of these actions can be seen in the improved Engineering Leadership Team drawing statistics for the SNF Project.

All four corrective actions for this finding have been completed.

FINDING: AMW-A-9901-F09

CLOSED

Facility component configuration or labeling was identified to either not match associated controlled drawings or not to be in accordance with conduct of operation matrix requirements.

The AP for labeling (AP EN-6-005) was revised to include guidance on using a graded approach in labeling. This was carried out to address effective resource management and ALARA considerations. Facility Engineering personnel were also required to complete a required reading assignment of procedures related to this subject. Facility Engineering then corrected noted labeling deficiencies in the identified drawings via two ECNs, and Operations made and hung the appropriate labels identified in three separate work packages.

All six corrective actions for this finding have been completed. 
SNF 4086, Rev 2

FDH-9953528A R3 Attachment

Appendix CM-2

Spent Nuclear Fuel Project

Closure/Status Report on Response FDH-9953258A R2

to RL Assessment 99-AMW-015

FINDING: AMW-A-9901-F10

CLOSED

Several issues were identified relative to an approved and issued work package authorizing installation and operation of a nuclear facility support system in a manner not consistent with an approved ECN.

\section{Finding 10A -}

IWTS work package 1K-97-2103 authorized installation of temporary power to a FRS panel board contrary to the approved ECN. Since the necessary engineering documentation was not prepared, an approval designator was not assigned and the modification was not unreviewed safety question (USQ) screened.

The necessary engineering documentation (ECNs and work instructions) was prepared, USQ screened, and approved per updated procedures and desk instructions. The power supply was placed in a permanent configuration, and when some unrelated work in this same work package is completed, the associated work package will be closed. The work instruction procedures and Construction Planning and Control desktop guide were updated to ensure that the appropriate development and approval steps are inherent in the process. In addition, the Systems Engineering Manager commissioned a review of the modified procedures and desktop guide to ensure the decision-making processes and instructions used in the field were consistent with applicable procedures.

All four corrective actions for this finding have been completed.

Finding $10 B$ -

No evidence was found that the NEC inspection required by step 7.18 .3 of construction work package $1 K-97-2103$ was completed as certified in the work package on January 22, 1999.

The Maintenance and Planning Managers reviewed the sign-off requirements from updated work guidance documents with all planning personnel to ensure that necessary approvals are in place before work is considered complete. In addition, the Projects organization reviewed a representative sample of other work packages and verified that approvals were being obtained per the updated work guidance documents. This included the review of the modification logs for ten separate work packages to ensure that they are in order and that all the proper signatures had been obtained in accordance with the revised desktop guide.

Both corrective actions for this finding have been completed. 
SNF 4086, Rev 2

Appendix CM-2

Spent Nuclear Fuel Project

Closure/Status Report on Response FDH-9953258A R2

to RL Assessment 99-AMW-015

\section{Finding 10C -}

Turnover and operation of the IXM flow meter installed by package $1 K-97-2103$ does not comply with the turnover requirements of HNF-PRO-572.

The Ion Exchange Module (IXM) flow meter configuration deficiency has been rectified by authorized updates to the appropriate documentation and the completion of all required fieldwork. The planners have been briefed on the modified procedures to prevent recurrences through reinforcing the expectation that effective inspection of all work planning documentation, including strict adherence to approval requirements, is required.

Corrective actions for Finding 10A completed the required actions to close this finding.

\section{FINDING: AMW-A-9901-F11}

\section{CLOSED}

Field changes using the modification log to path forward work packages are being authorized by project assigned construction project engineers vice documented cognizant engineers for the facility, as identified by facility work control procedure.

The seven work packages and associated modification log entries that were the subject of this finding have been reviewed against the updated Construction Planning and Control Desktop Guide to ensure that all necessary approvals are currently in place. The updated Desktop Guide also includes new definitions that will ensure the appropriate facility cognizant engineer signs off on all modification log entries. The work control procedures and Construction Planning and Control Desktop Guide were modified as part of the corrective actions for AMW-A-9901-F10(A).

The corrective action for this finding has been completed.

FINDING: AMW-A-9901-F12

CLOSED

Several approved DCNs and approved Requests For Information (RFI) which resulted in design changes for the CVDF Project were only partially included in the latest approved revision of the controlled drawings.

An assessment has been performed that evaluated facility drawings and DCNs to determine the status of incorporation of DCNs into project drawings. Identified deficiencies are being tracked via the DCN status log which is being reviewed and updated on a weekly basis. The CVD Facility DCN logs are kept current to allow the status of in-process and completed DCNs to be tracked. 
SNF 4086, Rev 2

Appendix CM-2

Spent Nuclear Fuel Project

Closure/Status Report on Response FDH-9953258A R2

to RL Assessment 99-AMW-015

Both corrective actions for this finding have been completed.

FINDING: AMW-A-9901-F13

CLOSED

Instances were identified in which submittals changed equipment and/or facility design without issuing an approved DCN to change the construction specifications as required.

The facility $\mathrm{A} / \mathrm{E}$ has completed a detailed review of over 800 submittals associated with the facility, and identified a total of seven submittals that affect the design, but which did not result in a DCN. These deficiencies are being corrected by a new DCN. The A/E's management has placed renewed emphasis on implementing proper change control and identification of all required changes. The CVD Facility management team is confident that the renewed focus on these issues specifically, and configuration management in general, will prevent this issue from recurring.

Both corrective actions for this finding have been completed.

\section{References:}

1. Letter, N. H. Williams, FDH, to J. M. Augustenborg, RL, "Contract Number DE-AC06-96RL13200 - Regarding Configuration Management Deficiencies on the Spent Nuclear Fuel Project," FDH-9953258 R2, dated July 2, 1999.

2. Letter, J. M. Augustenborg, RL, to R. D. Hanson, FDH, "Contract No. DE-AC06-96RL13200 - Configuration Management Deficiencies on the Spent Nuclear Fuel (SNF) Project," 99-AMW-015 / FDH-9953258A, dated May 10, 1999.

3. HNF-SD-SNF-CM-001, Revision 3, "SNF Configuration Management Plan," dated October 13, 1998.

4. Letter, L. L. Piper, RL, to R. D. Hanson, FDH, "Contract No. DE-AC06-96RL13200 -- Preliminary Notice of Violation and Proposed Imposition of Civil Penalty and Compliance Order EA-1999-04," 99-QSH-269 / FDH-9955895, dated August 19, 1999.

5. Letter, R. D. Hanson, FDH, to K. A. Klein, RL, "Transmittal of Project Hanford Management Contractor Activities for Enforcement Action EA-1999-04," FDH9953809A R38, dated October 14, 1999 
SNF 4086, Rev 2

Appendix CM-2

Spent Nuclear Fuel Project

Closure/Status Report on Response FDH-9953258A R2

to RL Assessment 99-AMW-015

\begin{tabular}{|c|c|}
\hline Table 1. Configuration Management Corrective Action Items & Status \\
\hline $\begin{array}{l}\text { C01-01: Strengthen the SNF Project CM Process. Identify configuration management-related problems and } \\
\text { then notify the SNF Project Systems Engineering Manager of identified problems for determination } \\
\text { of programmatic problems as noted below. Define a process: } \\
\text { - That will include a CM screening checklist to be used for the PIPs evaluation process } \\
\text { - Which notifies the SNF Project Systems Engineering Manager of all configuration management } \\
\text { issues } \\
\text { - To manage identified CM issues in order to evaluate for systematic/programmatic issues and take } \\
\text { appropriate actions }\end{array}$ & Complete \\
\hline \multicolumn{2}{|l|}{$\begin{array}{l}\text { C01-02: Strengthen the SNF Project self-assessment process to effectively monitor the Configuration } \\
\text { Management/engineering processes. }\end{array}$} \\
\hline 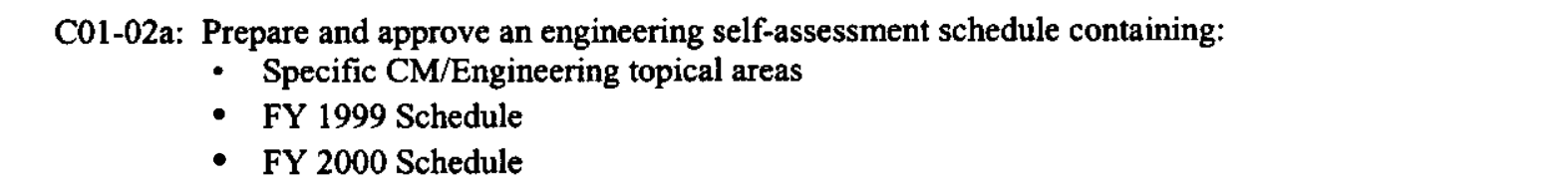 & Complete \\
\hline $\begin{array}{l}\text { C01-02b: Prepare FY } 1999 \text { assessment scope sheet, for each item on the self-assessment schedule, } \\
\text { identifying the following as a minimum: } \\
\text { - Scope of assessment } \\
\text { - Identification of applicable requirements }\end{array}$ & Complete \\
\hline $\begin{array}{l}\text { C01-02c: Perform surveillance on equipment labeling throughout the SNF Project by a sampling of various } \\
\text { subprojects and facilities. The surveillance will be based on Configuration Management, } \\
\text { engineering, and conduct of operations requirements. }\end{array}$ & Complete \\
\hline $\begin{array}{l}\text { C01-02d: Perform surveillance to assure that the Technical Document Control maintains design documents } \\
\text { in a controlled manner, per procedure, for the various } \mathrm{K} \text { Basin subprojects. }\end{array}$ & Complete \\
\hline $\begin{array}{l}\text { C01-02e: Incorporate funding into elements of the FY } 2000 \text { budget for continuing CM/Engineering } \\
\text { assessments which support the engineering assessment schedule. }\end{array}$ & Complete \\
\hline $\begin{array}{l}\text { C01-02f: Prepare FY } 2000 \text { assessment scope sheet, for each item on the assessment schedule noted in } \\
\text { Action C01-02a, identifying the following as a minimum: } \\
\text { - Scope of assessment } \\
\text { - Identification of applicable requirements }\end{array}$ & Complete \\
\hline $\begin{array}{l}\text { C01-02g: Perform surveillance of completeness of sets of past and current design documentation provided } \\
\text { by vendors and subcontractors participants. Assure that document control requirements have } \\
\text { been met. This will be a sampling of various subprojects throughout the SNF Project. }\end{array}$ & $\begin{array}{l}\text { Rescheduled } \\
\text { for FY00 }\end{array}$ \\
\hline C01-02h: Implement improved subcontractor and configuration control processes. & Complete \\
\hline $\begin{array}{l}\text { C01-02i: Perform assessment of SNF Project procedural controls to impose engineering processes } \\
\text { requirements on sub-tier contractors of engineering services. }\end{array}$ & Complete \\
\hline
\end{tabular}


SNF 4086, Rev 2

Appendix CM-2

Spent Nuclear Fuel Project

Closure/Status Report on Response FDH-9953258A R2

to RL Assessment 99-AMW-015

\begin{tabular}{|c|c|}
\hline Table 1. Configuration Management Corrective Action Items & Status \\
\hline $\begin{array}{l}\text { C01-02j: Conduct surveillance of sub-tier contractors of engineering services on both the engineering } \\
\text { processes and the compliance to these processes. }\end{array}$ & $\begin{array}{l}\text { Rescheduled } \\
\text { for FY00 }\end{array}$ \\
\hline C01-02k: Conduct surveillance on the implementation of the corrective action management process. & $\begin{array}{l}\text { Rescheduled } \\
\text { for } \\
\text { FY00 }\end{array}$ \\
\hline $\begin{array}{l}\text { C01-03: Communicate configuration management expectations throughout the SNF Project Engineering. } \\
\text { C01-03a: SNF Project Director to communicate a summary of the configuration management issues } \\
\text { identified by RL to SNF Project employees via e-mail. }\end{array}$ & Complete \\
\hline C01-03b: Briefing on change control and design verification expectations and requirements. & Complete \\
\hline C01-03c: Briefing on change control and design verification expectations and requirements. & Complete \\
\hline $\begin{array}{l}\text { C01-03d: Reinforce to the engineering staff the importance of rigorous compliance with engineering } \\
\text { change control procedures. }\end{array}$ & Complete \\
\hline $\begin{array}{l}\text { C01-04: Secure SNF Project resources necessary to provide infrastructure to ensure effective CM } \\
\text { implementation. }\end{array}$ & $\begin{array}{l}\text { FY00 } \\
\text { (Pending) }\end{array}$ \\
\hline $\begin{array}{l}\text { C01-05: Track and verify all corrective action (this plan). Monitor effectiveness of CM-related corrective } \\
\text { actions and identify additional areas of need. }\end{array}$ & $\begin{array}{l}\text { Rescheduled } \\
\text { for FY00 }\end{array}$ \\
\hline \multicolumn{2}{|l|}{$\begin{array}{l}\text { C02-01: Strengthen the SNF Project CAM Process. Effectively perform in a manner which provides resources } \\
\text { to identify, control, and uses a graded approach to determine cause and complete corrective actions. }\end{array}$} \\
\hline C02-01a: Define a process to evaluate new PIPs against existing PIPs to determine common issues. & Complete \\
\hline $\begin{array}{l}\text { C02-01b: Define a process that specifically requires trending of lower tier events in order to identify } \\
\text { significant issues and to prevent recurrence. }\end{array}$ & Complete \\
\hline $\begin{array}{l}\text { C02-01c: Define a process which incorporates configuration management screening, notification, and } \\
\text { evaluation activities consistent with the action in } 01-01 \text {. }\end{array}$ & Complete \\
\hline C02-01d: Define a process to verify corrective actions have been effective using graded approach. & Complete \\
\hline $\begin{array}{l}\text { C02-01e: Define a process that ensures SNF Project management involvement with work planning and } \\
\text { resource allocation to address identified corrective actions. }\end{array}$ & Complete \\
\hline C02-01f: Define a method to effectively manage the timeliness of the completion of corrective actions. & Complete \\
\hline C02-01g: Implement DTS and Corrective Action Management systems. & Complete \\
\hline C02-02: Estab & \\
\hline
\end{tabular}


SNF 4086, Rev 2

Appendix CM-2

Spent Nuclear Fuel Project

Closure/Status Report on Response FDH-9953258A R2

to RL Assessment 99-AMW-015

\begin{tabular}{|c|c|}
\hline Table 1. Configuration Management Corre & Status \\
\hline $\begin{array}{l}\text { C02-02a: SNF Project Director communicates to direct reports the corrective action management policy. } \\
\text { C02-02b: SNF Project Director direct reports review policy and related corrective action management } \\
\text { requirements with their staff. }\end{array}$ & $\begin{array}{l}\text { Complete } \\
\text { Complete }\end{array}$ \\
\hline C02-03: Perform assessment to ensure these corrective actions have been implemented correctly. & Complete \\
\hline $\begin{array}{l}\text { F01-01: Revise MHM Performance Specification HNF-S-0468. } \\
\text { F01-02: Management reemphasize that formal documentation is required for all verbal agreements that have } \\
\text { the potential to affect SNF Project facilities' configuration management. }\end{array}$ & $\begin{array}{l}\text { Complete } \\
\text { Complete }\end{array}$ \\
\hline $\begin{array}{l}\text { F02-01: Prioritize resources to process DCNs through construction organization in a more effective manner. } \\
\text { F02-02: Implement work control processes to ensure adequate supervision and critique of deficient work } \\
\text { processes. }\end{array}$ & $\begin{array}{l}\text { Complete } \\
\text { Complete }\end{array}$ \\
\hline $\begin{array}{l}\text { F02-03: Re-evaluate the problems and recurring nature of issues that were the subject of the March 4, } 1999 \\
\text { critique report including evaluation of January 26, } 1999 \text { NCR W-441-004. }\end{array}$ & Complete \\
\hline $\begin{array}{l}\text { F03-01: Allocate CVD Facility resources to complete root cause evaluation and needed actions to close PIPs } \\
\text { consistent with their significance. }\end{array}$ & Complete \\
\hline F03-02: Limit the ease in which CVD Facility PIPs' completion dates are extended. & Complete \\
\hline $\begin{array}{l}\text { F04-01: Enhance the Engineering Process Improvement Plan training activities to emphasize training for DAs } \\
\text { on interface control and enforcement of procedure requirements. } \\
\text { F04-02: Conduct surveillances on a sample basis to verify that ECNs with potential impact to interfaces meet } \\
\text { engineering process requirements. }\end{array}$ & Complete \\
\hline $\begin{array}{l}\text { F05-01: Stop practice of using RFIs for design changes. } \\
\text { F05-02: Use DCNs for implementing design changes. } \\
\text { F05-03: Review the RFIs used for design changes and develop an action plan to correct deficiencies. }\end{array}$ & $\begin{array}{l}\text { Complete } \\
\text { Complete } \\
\text { Complete }\end{array}$ \\
\hline $\begin{array}{l}\text { F06-01: Disposition remaining PIP } 97-389 \text { actions, conduct a } 100 \% \text { walk-down of the systems identified in } \\
\text { this PIP, and determine if other problems exist. } \\
\text { F06-02: Disposition remaining PIP } 98-161 \text { actions, conduct a } 100 \% \text { walk-down of the systems identified in } \\
\text { this PIP, and determine if other problems exist. }\end{array}$ & Complete \\
\hline $\begin{array}{l}\text { F06-03: Implement an ongoing engineering self-assessment process to measure the completeness and accuracy } \\
\text { of SNF Project essential and support drawings. }\end{array}$ & Complete \\
\hline F07-01: Revise appropr & Complete \\
\hline
\end{tabular}


SNF 4086, Rev 2

Appendix CM-2

Spent Nuclear Fuel Project

Closure/Status Report on Response FDH-9953258A R2

to RL Assessment 99-AMW-015

\begin{tabular}{|c|c|c|}
\hline & Table 1. Configuration Management Corrective Action Items & Status \\
\hline \multicolumn{3}{|c|}{$\begin{array}{l}\text { and issue memorandum to vendors and subcontractors, including the revised procedures within two } \\
\text { weeks of completing the procedure revisions. }\end{array}$} \\
\hline F08-01: & $\begin{array}{l}\text { Brief Plant Engineering staff on the need for and the requirements to incorporate ECNs (AP EN 6- } \\
\text { 039) }\end{array}$ & Complete \\
\hline F08-02: & $\begin{array}{l}\text { Communicate engineering management expectations to ensure controlled reassignment of personnel } \\
\text { conducting ECN work activities. }\end{array}$ & Complete \\
\hline $\begin{array}{l}\text { F08-03: } \\
\text { F08-04: }\end{array}$ & $\begin{array}{l}\text { Issue controlled support drawing list. } \\
\text { Schedule and dedicate engineering resources to ensure that essential and support drawings are } \\
\text { maintained. }\end{array}$ & $\begin{array}{l}\text { Complete } \\
\text { Complete }\end{array}$ \\
\hline F09-01: & $\begin{array}{l}\text { Issue response to RL Configuration Management Assessment Report (AMW-A-9901) thereby closing } \\
\text { S-98-WOD-KBasins-033-C01. }\end{array}$ & Complete \\
\hline F09-02: & Close A-98-WOD-KBasins-003-F13. & Complete \\
\hline F09-03: & $\begin{array}{l}\text { Field verify completion of labeling corrective actions of the specific deficiencies and thereby close A- } \\
98 \text {-WOD-KBasins-003-F14. }\end{array}$ & Complete \\
\hline F09-04: & Correct identified deficiencies in Essential Drawings $\mathrm{H}-1-51837$ sheet 3, and $\mathrm{H}-1-80550$ sheet 3. & Complete \\
\hline F09-05: & Revise AP EN 6-005/AP EN 6-028 to address graded approach to labeling. & Complete \\
\hline F09-06: & Document and implement graded labeling approach, provide required resources. & Complete \\
\hline F10-01: & $\begin{array}{l}\text { Investigate and resolve identified problems between the facility work processes and approved work } \\
\text { control procedures. }\end{array}$ & Complete \\
\hline F10-02: & $\begin{array}{l}\text { Bring the power supply to the IXM-4 flowmeter under configuration management control. This will } \\
\text { include appropriate ACW turnover actions. }\end{array}$ & Complete \\
\hline F10-03: & $\begin{array}{l}\text { Review the facilities' construction work control and decision-making processes to ensure that guides } \\
\text { and instructions used in the field are consistent with approved procedures. }\end{array}$ & Complete \\
\hline F10-04: & $\begin{array}{l}\text { Align the CP\&C Desktop Guide with AP MN 7-002, and } \\
\text { - Strengthen it to better define minor versus major work package changes } \\
\text { - Criteria definition and process of "Cognizant Engineer" } \\
\text { - Criteria for decision-making during field work processes } \\
\text { - Ensure that construction and maintenance understand that they are to contact engineering } \\
\text { whenever changes are proposed that will change facility configuration. }\end{array}$ & Complete \\
\hline F10-05: & Reinforce work package inspection step sign-off requirements with work planners. & Complete \\
\hline F10-06: & $\begin{array}{l}\text { Review a representative sample of work packages for required inspection documentation. Identify } \\
\text { corrective actions. }\end{array}$ & Complete \\
\hline
\end{tabular}




\section{FDH-9953528A R3 Attachment}

SNF 4086, Rev 2

Appendix CM-2

Spent Nuclear Fuel Project

\section{Closure/Status Report on Response FDH-9953258A R2}

to RL Assessment 99-AMW-015

Table 1. Configuration Management Corrective Action Items

F11-01: Conduct a review of the seven subject work packages modification log entries to confirm compliance with revised CP\&C desktop guide. This will be completed after the criteria from corrective action F10-04 is developed.

F12-01: Perform an assessment of CVD Facility drawings and DCNs to determine status of incorporation of DCNs into project drawings.

F12-02: Review the DCN status log to determine timelines and accuracy of the incorporation of DCNs into drawings.

F13-01: Perform a review to determine the extent of the problem.

F13-02: Disposition the issues of this finding.
Status

Complete

Complete

Complete

Complete

Complete 


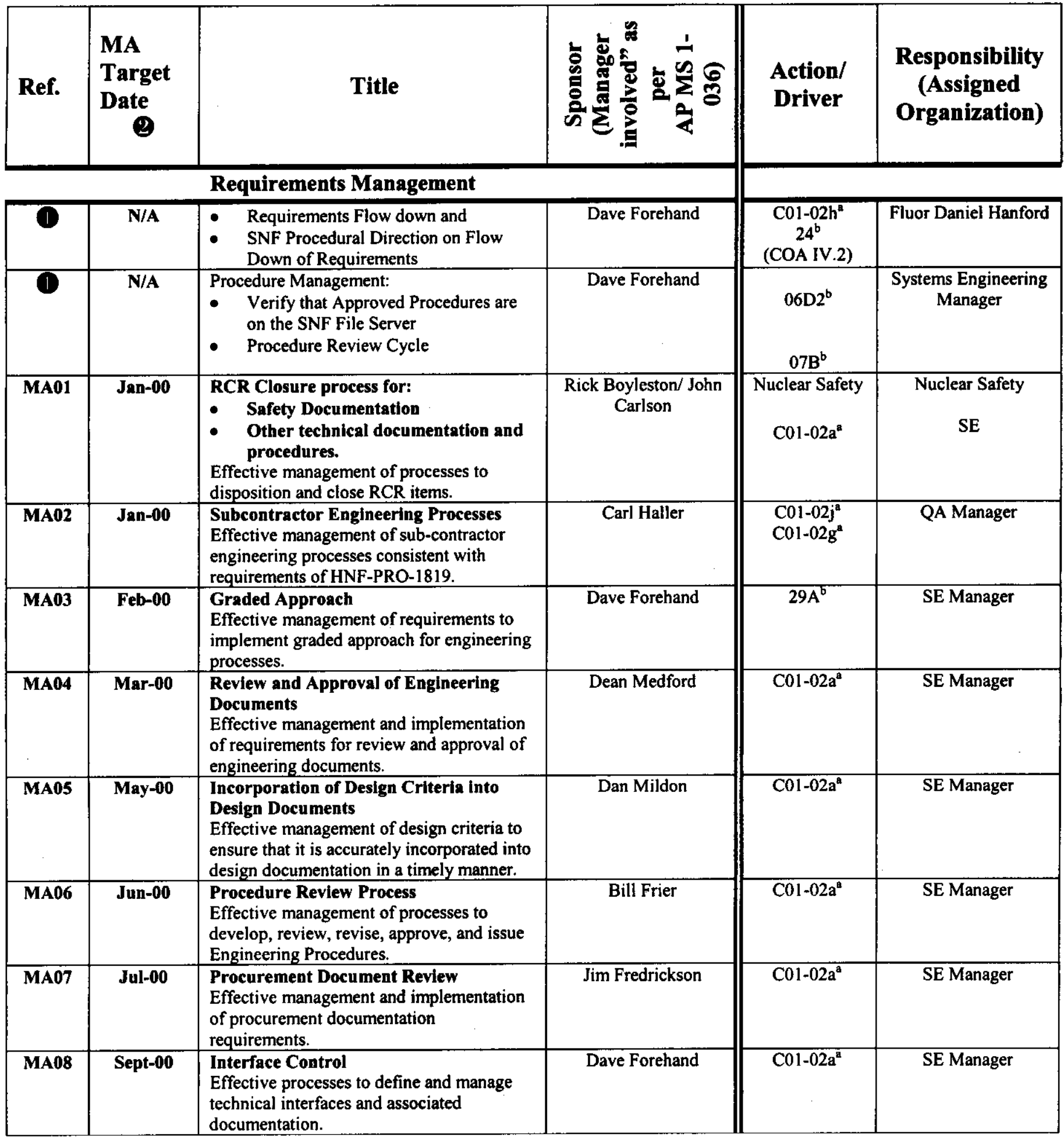

(1) Candidate assessment activity for the integrated schedule pending budget approval.

2) \#99-SNF/RBW-049, Spent Nuclear Fuel Project FY00 Management Assessment Plan Transmittal for commitment date.

${ }^{a}$ Response to 99-AMW-015 (FDH-9953258A R2),

${ }^{b} \mathrm{CM}$ Gap Analysis item 
SNF 4086, Rev 2

Appendix SA

Revision 990827 (status report 11-08-99)

SNF ENGINEERING SELF-ASSESSMENT SCHEDULE

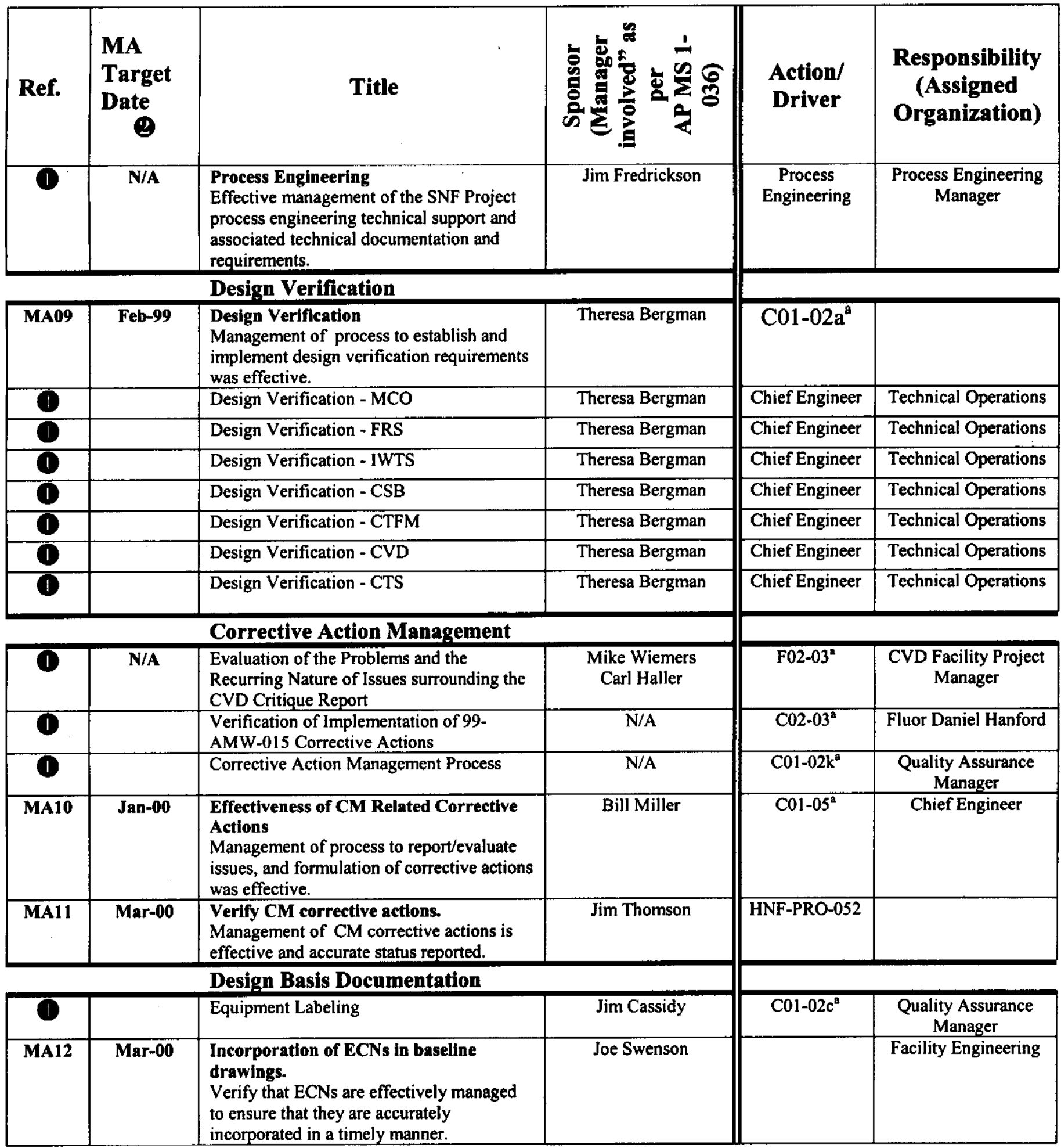

(1) Candidate assessment activity for the integrated schedule pending budget approval.

- \#99-SNF/RBW-049, Spent Nuclear Fuel Project FY00 Management Assessment Plan Transmittal for commitment date.

a Response to 99-AMW-015 (FDH-9953258A R2),

${ }^{b} \mathrm{CM}$ Gap Analysis item 
SNF 4086, Rev 2

Appendix SA

Revision 990827 (status report 11-08-99)

SNF ENGINEERING SELF-ASSESSMENT SCHEDULE

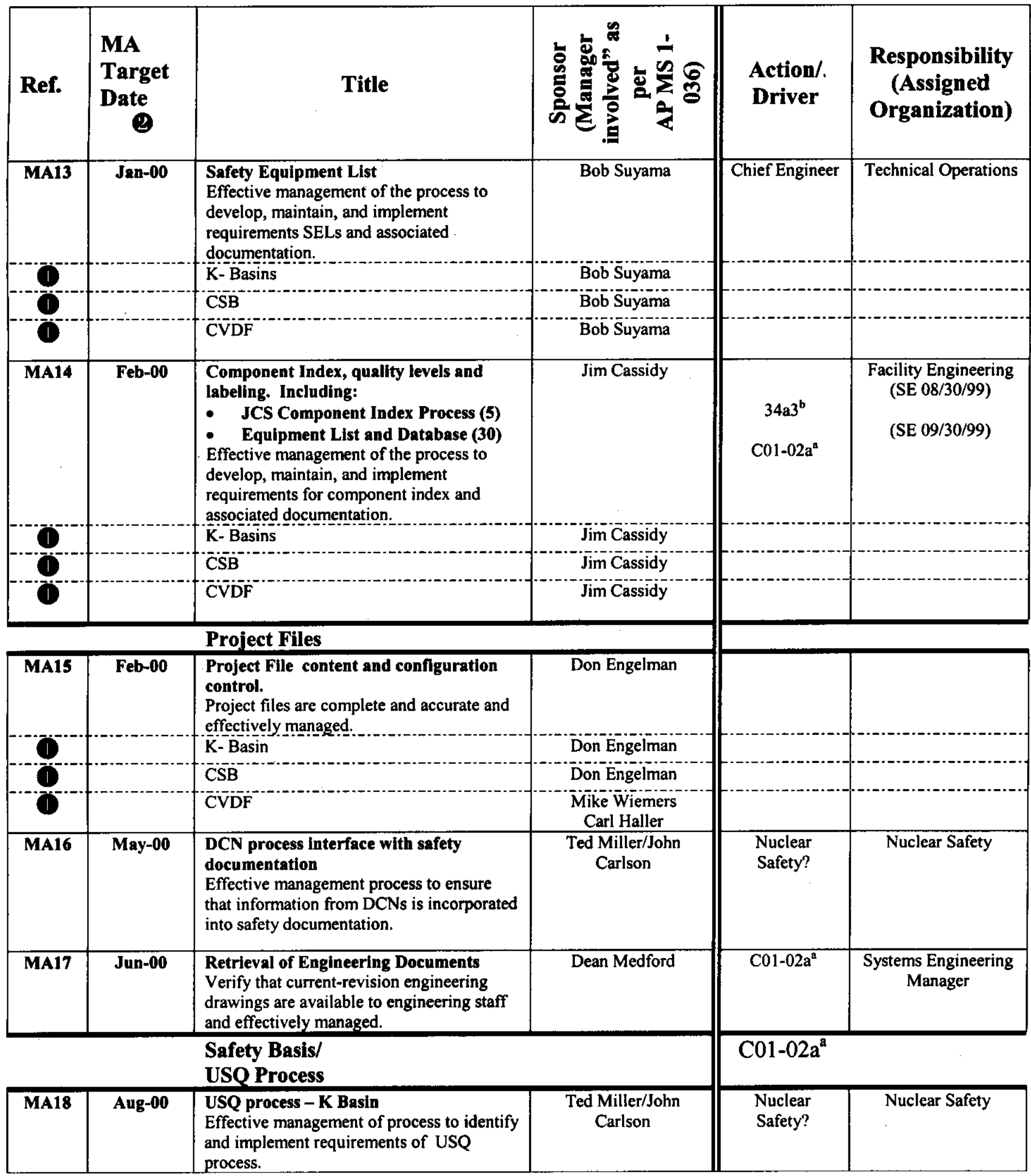

(i) Candidate assessment activity for the integrated schedule pending budget approval.

(2) \#99-SNF/RBW-049, Spent Nuclear Fuel Project FY00 Management Assessment Plan Transmittal for commitment date.

${ }^{\text {a }}$ Response to 99-AMW-015 (FDH-9953258A R2),

${ }^{\mathrm{b}} \mathrm{CM}$ Gap Analysis item 
SNF 4086, Rev 2

Appendix SA

Revision 990827 (status report 11-08-99)

SNF ENGINEERING SELF-ASSESSMENT SCHEDULE

\begin{tabular}{|c|c|c|c|c|c|}
\hline Ref. & $\begin{array}{l}\text { MA } \\
\text { Target } \\
\text { Date } \\
\quad 2\end{array}$ & Title & 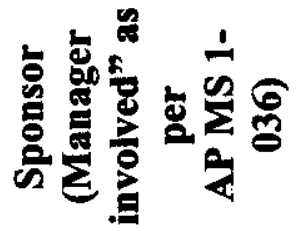 & $\begin{array}{l}\text { Action/ } \\
\text { Driver }\end{array}$ & $\begin{array}{l}\text { Responsibility } \\
\text { (Assigned } \\
\text { Organization) }\end{array}$ \\
\hline & & USQ process - new projects & $\begin{array}{l}\text { Ted Miller/John } \\
\text { Carlson }\end{array}$ & $\begin{array}{l}\text { Nuclear } \\
\text { Safety? }\end{array}$ & Nuclear Safety \\
\hline & & Nuclear Safety procedure implementation & John Carlson & $\begin{array}{l}\text { Nuclear } \\
\text { Safety? }\end{array}$ & Nuclear Safety \\
\hline & & $\begin{array}{l}\text { Nuclear Safety procurement documentation } \\
\text { review }\end{array}$ & Jack Garvin & $\begin{array}{l}\text { Nuclear } \\
\text { Safety? }\end{array}$ & Nuclear Safety \\
\hline \multicolumn{6}{|c|}{ Change Management } \\
\hline & & $\begin{array}{l}\text { Timelines and Accuracy of DCN } \\
\text { Incorporation }\end{array}$ & $\begin{array}{l}\text { Mike Wiemers } \\
\text { Carl Haller }\end{array}$ & $\mathrm{F} 12-02^{\mathrm{a}}$ & $\begin{array}{l}\text { CVD Facility Project } \\
\text { Manager }\end{array}$ \\
\hline & & $\begin{array}{l}\text { Assessment of ECN that potentially Impact } \\
\text { Interfaces }\end{array}$ & N/A & F04-02 ${ }^{\mathrm{a}}$ & $\begin{array}{c}\begin{array}{c}\text { Quality Assurance } \\
\text { Manager }\end{array} \\
\end{array}$ \\
\hline & & $\begin{array}{ll}\text { - } & \text { Design Change Notices } \\
\text { - } & \text { Startup Testing CM } \\
\text { Hanford Document Control System } \\
\text { status tracking } \\
\text { - Technical Document Control }\end{array}$ & Don Engelman & $\begin{array}{c}\text { C01-02a } \\
\text { Chief Engineer } \\
83 \mathrm{~B}^{\mathrm{b}}(8 / 30 / 99) \\
\\
\mathrm{C} 01-02 \mathrm{~d}^{\mathrm{a}} \\
(10 / 28 / 00) \\
\end{array}$ & $\begin{array}{l}\text { Systems Engineering } \\
\text { Manager }\end{array}$ \\
\hline & & Software Version Control & Jim Fredrickson & $\begin{array}{l}\text { Process } \\
\text { Engineering }\end{array}$ & $\begin{array}{l}\text { Process Engineering } \\
\text { Manager }\end{array}$ \\
\hline \multicolumn{6}{|c|}{ Work Controls } \\
\hline & & Work Package Inspection Documentation. & Bob Rasmussen & F10-06" & $\begin{array}{l}\text { K Basin Project } \\
\text { Manager }\end{array}$ \\
\hline & & $\begin{array}{l}\text { Work Package Modification Log Review } \\
\text { against CP\&C Desktop Guide }\end{array}$ & Bob Rasmussen & F11-01 ${ }^{2}$ & $\begin{array}{l}\text { K Basin Project } \\
\text { Manager }\end{array}$ \\
\hline
\end{tabular}

Candidate assessment activity for the integrated schedule pending budget approval.

(2) \#99-SNF/RBW-049, Spent Nuclear Fuel Project FY00 Management Assessment Plan Transmittal for commitment date.

${ }^{a}$ Response to 99-AMW-015 (FDH-9953258A R2),

${ }^{b} \mathrm{CM}$ Gap Analysis item 